\title{
Hybrid State Estimators for the Control of Remotely Operated Underwater Vehicles
}

\author{
by \\ Gregory Martin Vaughn \\ B.S., United States Naval Academy (1976) \\ Submitted in partial fulfillment of the \\ requirements for the degree of \\ OCEAN ENGINEER \\ at the \\ MASSACHUSETTS INSTITUTE OF TECHNOLOGY \\ and the \\ WOODS HOLE OCEANOGRAPHIC INSTITUTION \\ and \\ MASTER OF SCIENCE IN MECHANICAL ENGINEERING \\ at the \\ MASSACHUSETTS INSTITUTE OF TECHNOLOGY
}

August 1988

(c) Gregory M. Vaughn

The author hereby grants to MIT and WHOI permission to reproduce and to distribute copies of this thesis document in whole or in part.

Signature of Author

Joirlt Program in Oceanographic Engineering Massachtisetts Institute of Technology Woods Hole Oceanographic Institution August, 1988

Certified by

Dr. Dana R. Yoerger

Woods Hole Oceanographic Institution

Thesis Supervisor

Certified by

Dr. Jean-Jacques E. Slotine Massachusetts Institute of Technology

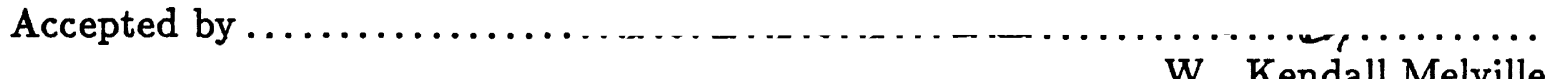

Chairman, Joint Committee for Oceanographic Engineering, Massachusetts, Institute of Technology/Woods Hole Oceanographic Institution 


\title{
Hybrid State Estimators for the Control of Remotely Operated
}

\author{
Underwater Vehicles \\ by \\ Gregory Martin Vaughn
}

\author{
Submitted to the Massachusetts Institute of Technology- \\ Woods Hole Oceanographic Institution \\ Joint Program in Oceanographic Engineering \\ on August, 1988, in partial fulfillment of the \\ requirements for the degree of \\ OCEAN ENGINEER \\ and \\ MASTER OF SCIENCE IN MECHANICAL ENGINEERING
}

\begin{abstract}
This paper explores the use of 'hybrid' state estimators to increase the accuracy and flexibility of acoustic position measurement systems used in the control of underwater vehicles. Two different approaches to extend the range of acoustic position measurement systems are explored. The first approach is the use of an inexpensive Strapdown Inertial Measurement System (SIMS) to augment the acoustic with inertial position information. This approach is based on the experience gained using an attitude and inertial measurement package fielded on the JASON JUNIOR Remotely Operated Vehicle (ROV). The second approach is the use of a mobile, platform-mounted, acoustic net in conjunction with a platform tracking system. This second investigation used the JASON ROV as the basis for the simulation work. As motivation, some of the theoretical and practical difficulties encountered when range is extended using an unaugmented system are explored. Simulation results are used to demonstrate the effects of these difficulties on position estimation accuracy and on the performance in closed loop control of the vehicle. Using measured sensor noise characteristics, a hybrid Kalman filter is developed for each approach to take the greatest advantage of the available information. Formulation of the Kalman filter is different for each case. In the second case, the geographic position of the ROV is the sum of the acoustic net's geographic position, measured at a different interval by an $\mathrm{RF}$ positioning system, and the position of the ROV relative to the net, as measured acoustically. Closed loop vehicle performance evaluations are made for representative noise levels and update rates with and without the augmentation discussed in the first approach. Finally, conclusions are drawn about the benefits and applications of the hybrid Kalman filter to the control of Remotely Operated Vehicles.
\end{abstract}

Thesis Supervisor: Dr. Dana R. Yoerger

Woods Hole Oceanographic Institution 


.




\section{Acknowledgements}

This dissertation was completed with the help and encouragement of many people and several organizations. I would like to mention a few of them.

I am deeply indebted to my wife, Susan, without whose love and unwavering support during the coursework at MIT and the separations during my research at Woods Hole, I would not have been able to complete this project.

I would like to thank Dr. Dana Yoerger, my thesis advisor, who deserves much credit for leading me to my topic and deserves much thanks for his untiring enthusiasm in support of my work at the Deep Submergence Lab. I also owe a great deal to my fellow students at the lab who have supported me in my expeditions into the ' $C$ ' programming language.

For his insight into Kalman filtering, nonlinear control, and for his support of my research, I thank Dr. Jean-Jacques E. Slotine at MIT.

I would like to thank Dr. Arthur Baggeroer and the MIT/WHOI Joint Program staff who assisted me in many ways during my navigation through the Joint Program. I include in this group the Education Office Staff at Woods Hole.

Financially, I am indebted to the Oceanographer of the Navy for support I received in pursuing these degrees.

Finally, I am most grateful to the NAVY for permitting me to take advantage of such a tremendous opportunity in graduate education at this point in my career. 


\section{Contents}

1 Introduction $\quad 11$

1.1 Motivation ........................... 11

1.2 Incorporation of Inertial Acceleration Measurements . . . . . . . . . 11

1.2.1 Potential Advantages in ROV Control . . . . . . . . . . . . 11

1.2.2 Inertial Measurement Mechanization . . . . . . . . . . . 12

1.2 .3 Sensor Integration $\ldots \ldots \ldots \ldots \ldots \ldots$

1.3 Use of a Moving Acoustic Position Tracking Net . . . . . . . . . . . . . 14

1.3.1 Desirable Features .................... 14

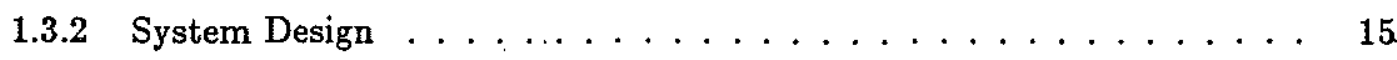

1.4 Simulation Guidelines $\ldots \ldots \ldots \ldots \ldots \ldots$

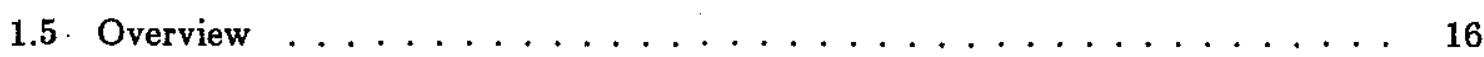

2 Underwater Vehicle Dynamics $\quad 19$

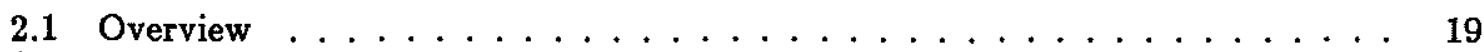

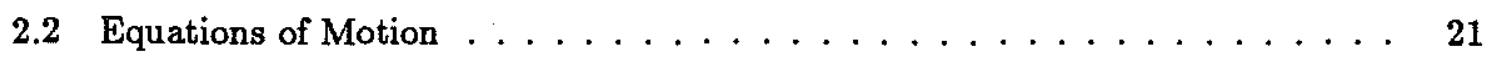

2.2.1 Equations for a Single Degree of Freedom . . . . . . . . . . . 21

2.2.2 Equations for Six Degrees of Freedom . . . . . . . . . . . . . 24

2.3 Dynamic Distinctions between JASON and JASON JUNIOR . . . . . . . 28

2.3.1 Dynamics of JASON JUNIOR . . . . . . . . . . . . . 28

2.3 .2 JASON Dynamics $\ldots \ldots \ldots \ldots \ldots$

3 Discrete Linear Observers in Underwater Vehicle Control 34

3.1 Closed Loop Versus Open Loop Control . . . . . . . . . . . . . . . . . 34

3.2 Full State Feedback and Selection of ROV Dynamics . . . . . . . . . . . 36 
3.2.1 Full State Feedback Structure . . . . . . . . . . . . . 36

3.3 Discrete Implementation of Full State Feedback . . . . . . . . . . . 36

3.3.1 Transformation to Discrete Time Representation . . . . . . . . 37

3.3.2 Sampling Rate Selection . . . . . . . . . . . . . . 38

3.4 Limitations of Full State Feedback . . . . . . . . . . . . . . . 39

3.4 .1 Low Update Rates . . . . . . . . . . . . . . . . . 39

3.4 .2 Noisy or missing measurements . . . . . . . . . . . 39

3.4.3 Steady State Error . . . . . . . . . . . . . . . 41

3.5 Full Order Observers . . . . . . . . . . . . . . . . . . 42

3.5.1 Discrete-Time Observer Structure . . . . . . . . . . . 42

3.5 .2 Observability . . . . . . . . . . . . . . . 42

3.5.3 Observer and Error Dynamics . . . . . . . . . . . . . . 44

3.5.4 Stochastic Considerations in Observer Design . . . . . . . . . . 45

3.5.5 Optimal Linear Observers: Kalman Filters . . . . . . . . . . 47

3.5 .6 Kalman Filter Dynamics . . . . . . . . . . . . . . 48

3.5.7 Steady State Kalman Filter . . . . . . . . . . . . . . 49

4 Utilization of Strapdown Inertial Measurements for JASON JUNIOR 50

4.1 Inertial Navigation System Basics . . . . . . . . . . . . . 50

4.1 .1 Coordinate Frames . . . . . . . . . . . . . . 52

4.1 .2 Inertial Sensors . . . . . . . . . . . . . . 53

4.2 Strapdown Inertial Measurement Systems (SIMS) $\ldots \ldots \ldots \ldots \ldots$

4.2.1 How SIMS is different . . . . . . . . . . . . . 56

4.2 .2 Hardware and Sensors . . . . . . . . . . . . . 56

4.2.3 Attitude Determination . . . . . . . . . . . . . 59

4.2.4 Removing the Gravity Vector . . . . . . . . . . . . . 60

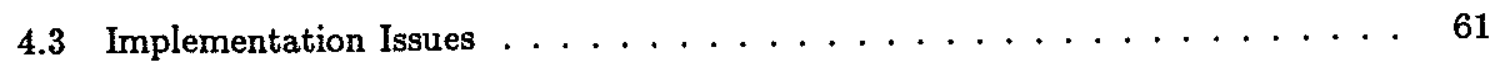

4.3 .1 Physical Size . . . . . . . . . . . . . . . 61

4.3.2 Calculational Complexity .................... 61

4.4 Sources of Error in SIMS Output . . . . . . . . . . . . . 61

4.4.1 Errors Due to Algorithm Selection ............... 61 
4.4 .2 Sensor Resolution $\ldots \ldots \ldots \ldots 2$

4.4.3 Calibration and Alignment Errors .............. 62

4.4 .4 Gyro Drift . . . . . . . . . . . . . . . 63

4.4.5 Gravity Vector Anomalies . . . . . . . . . . . . . . . 63

4.4.6 Approximations and Numerical Uncertainty . . . . . . . . . . . 63

5 Hybrid State Estimation for JASON JUNIOR

5.1 Objectives for the State Estimator on JASON JUNIOR . . . . . . . . 65

5.1 .1 Redundancy in Measurements . . . . . . . . . . . . . 65

5.1 .2 Improved Estimator Dynamics . . . . . . . . . . . 66

5.1 .3 Long Term Estimation . . . . . . . . . . . . . . . 67

5.2 Available State Information for JASON JUNIOR . . . . . . . . . . 67

5.2.1 Acoustic Position Information . . . . . . . . . . . . 67

5.2 .2 Strapdown Acceleration Measurements . . . . . . . . . . . . 67

5.3 Use of the 'Hybrid' JASON JUNIOR Observer . . . . . . . . . . . . 68

5.3.1 Preliminary Considerations . . . . . . . . . . . . . 69

5.3.2 Development of the 'Hybrid' Observer . . . . . . . . . . . 70

5.3.3 Simulation Results . . . . . . . . . . . . . . . 71

6 Hybrid State Estimator for ROV JASON $\quad 81$

6.1 Operational Scenario . . . . . . . . . . . . . . . . 81

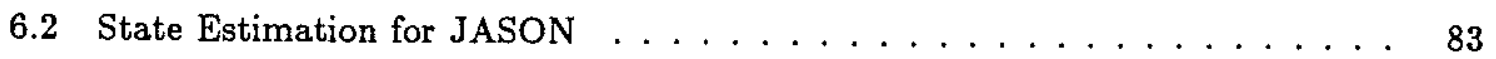

6.2.1 Role of the State Estimator . . . . . . . . . . . . 83

6.2 .2 Objectives of the State Estimator . . . . . . . . . . . 83

6.3 Available State Measurements . . . . . . . . . . . . . . . . . 84

6.3.1 RF Navigation System Measurements . . . . . . . . . . . . 84

6.3.2 Mobile Acoustic Positioning System . . . . . . . . . . . 85

6.3.3 The Research Vessel . . . . . . . . . . . . . . . . 86

6.4 Attitude Determination of the Research Vessel . . . . . . . . . . 86

6.4 Pitch and Roll Effects . . . . . . . . . . . . . . 86

6.4 .2 Attitude Sensors . . . . . . . . . . . . . . 88 
6.4 .3 Vessel Attitude Calculation . . . . . . . . . . . . 89

6.5 Two Stage Kalman Filter Development . . . . . . . . . . . . . 90

6.5.1 Kalman Filter Assumptions . . . . . . . . . . . . . . 91

6.5.2 Two-Stage Kalman Filter Design . . . . . . . . . . . . . . 93

6.6 Simulation Design . . . . . . . . . . . . . . . . . . 94

6.7 Two Stage Kalman Filter Tracking Simulation Results . . . . . . . . . . 95

6.8 Conclusions . . . . . . . . . . . . . . . . . 103

7 Conclusions $\quad 104$

7.1 Achievements Versus Objectives . . . . . . . . . . . . . . 104

7.2 Control Bandwidth Improvements . . . . . . . . . . . . . . . 104

7.2.1 Bandwidth Effects on JASON JUNIOR . . . . . . . . . . . . 104

7.2.2 The Effects of the JASON 'Hybrid' Estimator . . . . . . . . . . 105

7.3 Refinements and Improvements . . . . . . . . . . . . . 105

7.3.1 Strapdown Inertial Measurement Systems . . . . . . . . . . . 105

7.3.2 Mobile Acoustic Nets . . . . . . . . . . . . . 106

7.4 Remaining Work . . . . . . . . . . . . . 106

7.4.1 Control Developments . . . . . . . . . . . . 107

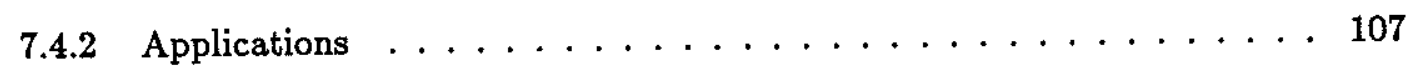

7.5 Closing Remarks . . . . . . . . . . . . . . . . . 108 


\section{List of Figures}

1-1 Effects of Changing Sensor Update Rate . . . . . . . . . . . . 18

2-1 The ROV Used to Demonstrate Nonlinear Closed Loop Control (RPV). . . 20

2-2 $C_{d r a g}$ versus $R e$ for a rough surface sphere submerged in water. . . . . . . 23

2-3 Illustration of an ROV's Six Degrees of Freedom $\ldots \ldots \ldots \ldots$

$2-4$ Major Features of ROV JASON JUNIOR. . . . . . . . . . . . . . . . 29

2-5 Operation Deployment of ROV JASON JUNIOR. . . . . . . . . . . . . 31

$2-6$ Three Dimensional View of ROV JASON. . . . . . . . . . . . . . 32

3-1 Time Domain Block Diagrams of Typical Open and Closed Loop Systems. . 35

3-2 The Destructive Effects of Sensor Noise on Closed Loop Performance . . . . 40

3-3 Effect of Noisy Measurements on Closed Loop Control Actions . . . . . . . . 41

3-4 Block Diagram of a Discrete Time, Full Order Linear Observer. . . . . . . . . 43

3-5 Sensor Noise Distribution for Acoustic Positioning System . . . . . . . . . 46

3-6 Sensor Noise Distribution for Del Norte RF Position System. . . . . . . . . . 47

3-7 Single Stage Discrete Kalman Filter Flow Path . . . . . . . . . . . . . 48

4-1 Integrated Accelerometer and Attitude Sensor Package for JASON JR. . . . . 51

4-2 Basic Block Diagram of a Stabilized Inertial Navigation System . . . . . . . . 52

4-3 Conceptual Block Diagram of the Strapdown Inertial Navigation System. . . 53

4-4 Euler Angles and their Associated Rotations. . . . . . . . . . . . 54

4-5 Photograph and Basic Block Diagram of Torque-Balanced Linear Accelerometer. 58

5-1 Sonic, High Accuracy, Range and Positioning System . . . . . . . . 68

5-2 JASON JUNIOR 'Hybrid' Observer Flow Path. . . . . . . . . . . . . 72 
5-3 Inertial Reset Logic Tree. . . . . . . . . . . . . . . 76

5-4 JASON JUNIOR 'Hybrid' State Estimator Simulation Results: Case 1. . . . 77

5-5 JASON JUNIOR 'Hybrid' State Estimator Simulation Results: Case 2. . . . 78

5-6 JASON JUNIOR 'Hybrid' State Estimator Simulation Results: Case 3. . . . 79

5-7 JASON JUNIOR 'Hybrid' State Estimator Simulation Results: Case 4. . . . 80

6-1 Integrated Employment of RF and Acoustic Positioning Systems . . . . . 82

6-2 Typical Employment of an RF Positioning System . . . . . . . . . . 84

6-3 The Research Vessel McGaw. . . . . . . . . . . . . . . 87

6-4 The Affects of Vessel Pitch on Position Information. . . . . . . . . . . 88

6-5 The Affects of Vessel Roll on Position Information. . . . . . . . . . . . . 89

6-6 Plot of the Integrated Inclinometer Frequency Response . . . . . . . . . . 90

6-7 Alternate Flow Path for a Two Measurement Kalman Filter. . . . . . . . . . 92

6-8 Simulation Results of Hybrid State Estimation for JASON and SHIP. . . . . 96

6-9 Simulation Results with Initial SHIP Position Error $\ldots \ldots \ldots$. . . . . . 97

6-10 Simulation Results with Initial ROV Position Error $\ldots \ldots \ldots$. . . . . . . 98

6-11 Combined Geographic Position Estimation Error for JASON and SHIP. . . . 100

6-12 Simulation Error Plot with SHIP Initial Condition Error . . . . . . . . 101

6-13 Simulation Error Plot with ROV Initial Position Error . . . . . . . . . . . . 102 


\section{List of Tables}

4.1 Resolution and Error Specifications of SIMS Sensors. . . . . . . . . . . . 62

6.1 List of Different Simulation Runs . . . . . . . . . . . . . . . 95

6.2 Summary of Simulation Results. . . . . . . . . . . . . . . . 99

6.3 Results of Additional Simulation Runs. . . . . . . . . . . . . . . . . . 99 


\section{Chapter 1}

\section{Introduction}

\subsection{Motivation}

Underwater remotely operated vehicles (ROV's) can be made more useful and easier to operate if their movements are automatically controlled. Such automatic position control provides the basis for either supervised or autonomous operation.

In most applications where traditional acoustic navigation techniques are employed [1], the closed loop performance will be strongly limited by the imprecision and limited update rate of the position reference. In this thesis the development and use of application specific state estimators is investigated as a systematic approach to combining additional sensor information and increasing the effective control bandwidth of the Remotely Operated Vehicle (ROV).

Two different approaches to incorporating additional sensor information are explored in this paper. The first is the use of inertial acceleration measurements and the second is the combination of short range and long range position measurements. Both approaches are aimed at increasing the effective control bandwidth of remotely operated underwater vehicles.

\subsection{Incorporation of Inertial Acceleration Measurements}

\subsubsection{Potential Advantages in ROV Control}

The measurement of inertial accelerations can be useful in two important ways.

1. First, inertial sensing can be valuable in achieving larger control bandwidths. In conventional acoustic systems, limitations are imposed by inherent travel time delays. Inertial 
measurement units can update vehicle state information at a rate which is independent of actual state and will experience a much smaller delay in long range applications.

2. Second, inertial state information can assist existing noise rejection techniques by providing redundant state measurements. This can improve control by providing a lower overall noise level in the position estimate. Acoustic navigation systems will also benefit from the redundant measurements by being able to remove random bad fixes, or 'flyers', without suffering the loss of position measurement during that interval.

Both of these goals are best accomplished by combining the information in a state estimator or observer. The exact nature of the most appropriate state estimator for an Underwater ROV is based on the specific ROV, the available sensors, and its intended application.

A simple simulation using the equations developed in Chapter 2 graphically illustrates the effects of low update rates. Modeling error has been introduced by using a linearized model for the observer and a nonlinear simulation utilizing a fourth order Runge-Kutta [2] approximation to simulate the vehicle. The errors increase dramatically as the time between updates increases - even without the presence of noise in the measurements. The results of this simulation are presented as figure (1-1).

Even with an observer to provide state estimates in the absence of actual measurements, low update rates can have several deleterious effects. Most of these effects are outgrowths of the fact that models of the system are inherently inaccurate, especially when a linear model is used for a nonlinear plant [3]. The penalties paid for the lower update rates are usually:

- much larger control actions, which may saturate the actuators, and

- reduced accuracy resulting from the presence of hidden dynamics.

Chapter 3 explores the effects of various bandwidths on system performance in more detail.

\subsubsection{Inertial Measurement Mechanization}

While inertial measurement systems are highly developed and have been used for navigation on board aircraft, spacecraft, submarines, and missiles, they have only relatively recently been of a 'strapdown' variety [4]. 
Inertial measurement systems in common use have principally been complex, stabilizedplatform systems. This is because they have often been the primary, if not the only, navigation system their associated vehicles relied upon over relatively long periods of time. Because of their expense, power requirements, and size, however, the use of these complex inertial guidance and navigation systems has been considered inappropriate for most tethered underwater vehicles and for all but the most ambitious of autonomous underwater vehicle projects. The strapdown sensor package used in these experiments is quite small and has minimal power requirements.

Small, remotely operated underwater vehicles, like JASON JUNIOR at the Deep Submergence Laboratory of the Woods Hole Oceanographic Institution, might benefit from a strapdown inertial measurement system augmenting an existing navigation system. Small size and weight is important to JASON JUNIOR because of its own diminutive profile. Low power is important to JASON JUNIOR because high power requirements would require a larger tether which translates into higher drag on the vehicle. An added benefit of a small sensor package to a vehicle used in deep submergence applications is the reduced size and expense of the watertight enclosure required to withstand full submergence pressure. (JASON JUNIOR has been tested to 6000 meters and was used at 4000 meters in the 1986 exploration of the RMS Titanic.)

\subsubsection{Sensor Integration}

The navigation system currently in use on JASON JUNIOR is a high frequency acoustic position tracking system.

The raw output of a Strapdown Inertial Measurement System (SIMS) is a set of three accelerations relative to a body-fixed coordinate frame and body referenced attitude measurements. The three accelerations are contaminated by the gravity vector and also contain radial accelerations which result from the displacement of the sensors from the vehicle's center of mass. It is possible, with the help of vehicle attitude information, to extract the vehicle's geographic position and velocity from the raw accelerations. We can either use vehicle velocity directly to implement full state feedback for the controller or use inertial position to provide redundancy for the acoustic position information in order to reduce the noise in the 
position estimate. The latter approach would use estimated states for feedback.

The use of the existing acoustic positioning system with the SIMS is roughly analogous to the use of periodic satellite navigation fixes to update the more complex stable-platform inertial navigation systems. The difference in the application of a SIMS on an ROV is that the position fixing system is actually the primary navigation sensor. The acoustic position fixing system would be augmented by inertial information during relatively long sampling intervals or in particularly noisy environments. Long sampling intervals are required in medium range (long acoustic baseline) applications where the acoustic travel time introduces a pure delay in the position information. As an example, travel time for a straight line distance of 1000 meters is .667 seconds. Although techniques can be used to increase the apparent update rate (e.g. range-gating, frequency modulation) the delay experienced cannot be recovered and reduces the control bandwidth.

\subsection{Use of a Moving Acoustic Position Tracking Net}

\subsubsection{Desirable Features}

Recognizing the inherent difficulties of long range acoustic position measurements, another approach is to maintain the range from the transducers (on a reference platform, for example) to the ROV short enough that the position update rate is not the limiting factor in vehicle control bandwidth. If off the shelf systems were available to track the reference platform within acceptable accuracy limits, this might relieve the burden on the scientist to build, maintain, program or service one of a kind inertial measurement packages. On the other hand, an existing inertial package could offer redundancy for such a capability to support noise rejection algorithms.

As an alternative to the deployed acoustic nets, a mobile net operating within a wider area net may mean more effective resource utilization as less time is wasted in deploying, retrieving, and redeploying the net. What makes this larger net concept attractive is that it can be accomplished with an RF system which has good precision and no appreciable delay. 


\subsubsection{System Design}

This system concept might be implemented by having the high accuracy (high frequency) acoustic net rigidly attached to a mother ship whose position is monitored by an RF positioning system. The geographic position of the ROV then becomes a linear combination of the ship's position relative to the RF net and the ROV's position relative to the ship (acoustic net.) The mother ship is kept within that radius of the vehicle necessary to keep acoustic travel times within acceptable limits.

This approach is not without its difficulties, however. One problem is that typical accuracies of $\mathrm{RF}$ positioning systems are an order of magnitude lower than those of the acoustic positioning systems tested. (A comparison between the RF and acoustic measurement system noise can be found in Chapter 3.) But, because of the RF system's relatively high update rate and relative to the sluggish dynamics of the large mother ship it can be shown that the ship's position information can undergo relatively heavy filtering without introducing significant phase distortion. This filtering is best accomplished with an observer designed to minimize position error in an appropriate way. Again the objective is to establish closed loop control of the geographic position of the ROV over a relatively large area with a minimum of error.

Another systemic problem is the effect of ship's pitch and roll on the acoustic measurements of vehicle position and to a much smaller extent, the RF position measurements of the ship. Although the ship will be a relatively stable platform, an accurate measurement of the attitude of the ship is required to remove the pitch and roll effects. This is extremely important to achieve a similar degree of accuracy as a fixed acoustic position measurement system.

\subsection{Simulation Guidelines}

Both of the approaches presented above, as well as a combination of the two will be treated in the simulations presented in this paper. The bases for the simulations contained in this report are:

1. Actual implementation of an inertial measurement/attitude package which has been installed and tested on JASON JUNIOR. 
2. Operational experience gained in using the Sonic High Accuracy Ranging and Positioning System (SHARPS).

3. Operational experience gained in using the DEL-NORTE RF positioning system.

4. Operating experience with the ROV JASON JUNIOR.

To the maximum extent possible, these simulations are the extension of actual experiments and their data. The data gathering and control algorithms for the simulations and actual experiments with the vehicle are written in the $\mathrm{C}$ programming language and have been implemented on a personal computer.

In general then, calculations and simulations performed and reported in this thesis use the parameters and nominal values associated with actual implementations. Where available, real data is presented.

\subsection{Overview}

In Chapter 2, the linear modeling and dynamics associated with remotely operated vehicles, specifically JASON JUNIOR, are presented. The modelling performed here is used in the following chapters to construct the algorithms necessary for state estimation for the ROV. Differences in dynamics between ROV JASON and JASON JUNIOR are highlighted.

Chapter 3 contains a development of full order linear observers. The full order or 'identity' observer forms the framework on which to hang the more specific discussions of noise rejection, filtering and ROV state estimation also covered in this section. A short discussion of the stochastic properties of noise which is characterized by gaussian probability distributions is included along with a discussion of discrete Kalman Filters. This background material is tailored specifically to the ROV application.

In Chapter 4 the basics of an inertial navigation system are sketched out and a more in depth discussion of strapdown systems is presented. The discussion of the strapdown systems includes an examination of the sensors utilized on the actual package fielded on the JASON JUNIOR ROV. An examination of the specific implementation issues associated with the inertial sensing and attitude measurement package is included. An evaluation of the probable sources of error is made here. 
Chapter 5 combines the common elements of chapters 2 through 4 in the development and simulation of a 'hybrid' state estimator. By 'hybrid' state estimation, we refer to one which combines the inertial sensing package information with the acoustic position tracking system output. Errors in position and control using varying acoustic position update rates are measured with and without the inertially augmented state estimator. JASON JUNIOR is the testbed for this estimator.

Chapter 6 is an exploration of the alternate approach to longer periods between acoustic positions; the use of an RF position tracking system in conjunction with a moving acoustic net. This represents an alternative 'hybrid' state estimator. An in depth development of the state estimator is presented. Implementation issues for the JASON ROV are also explored in some detail. Simulation results obtained using such a hybrid state estimator are displayed in graphical and tabular form. Some conclusions from the simulation results are drawn.

Chapter 7 presents a summary of the results obtained in the previous chapters and outlines the shortfalls associated with the approaches examined. A discussion of measures which may improve and refine the ROV state estimation strategies provides the basis for additional research. 


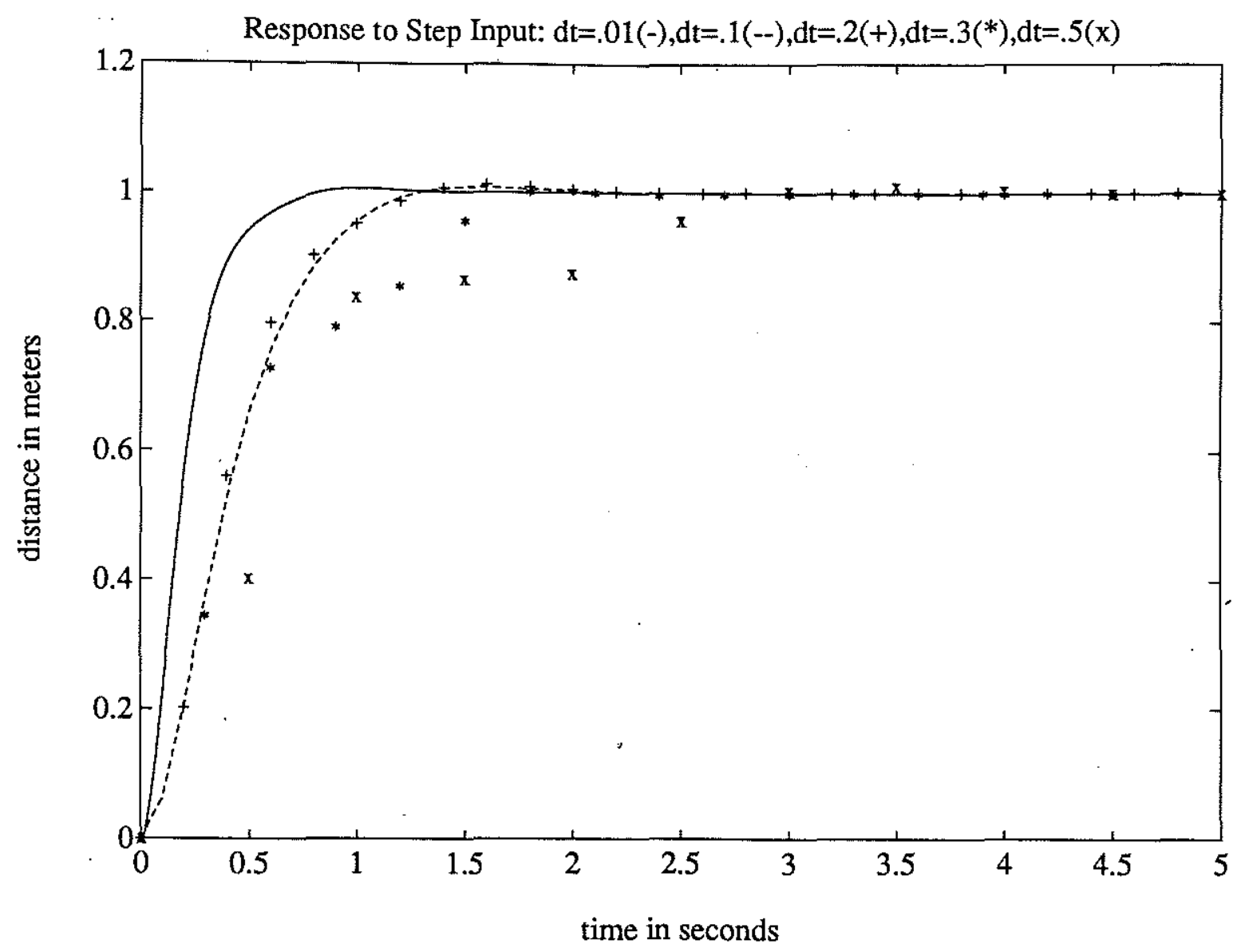

Figure 1-1: A series of simple computer simulations which illustrate the deleterious effects on ROV performance when sensor update rate is lowered and yet still meets the Nyquist criteria. If control action were clipped at levels corresponding to thruster capacity, or measurement noise were introduced, performance degradation would have been even more dramatic. 


\section{Chapter 2}

\section{Underwater Vehicle Dynamics}

\subsection{Overview}

A clear understanding of vehicle dynamics is essential to the successful implementation of vehicle state estimators and attitude tracking algorithms. A linear description of the dynamics is complicated by the environment in which an ROV operates. This hydrodynamic environment results in decidedly nonlinear vehicle behavior. In recognition of the hydrodynamic complexities, this chapter presents a nonlinear development of the dynamics first, utilizing the hydrodynamic descriptions as best we understand them. A tangent linearization follows the development of the nonlinear equations of motion. (Tangent linearization is simply a Taylor Series expansion of the equations about our desired operating point or set of points.)

Although a successful implementation of nonlinear, closed-loop (sliding surface) control of an ROV has been demonstrated [3], it relied on essentially noise-free, relatively high speed, position sensors. Similar sensors are not currently available for long range tasks. Yoerger's experiments were conducted in a test pool with an average distance from vehicle (transmitter) to sensor (receiver) of approximately 15 meters. Figure (2-1) is a drawing of the vehicle use in these tests. Because a combination of data from multiple noisy sensors is envisioned, a linear approach to the state estimation problem has been chosen to take advantage of the vast body of knowledge available in linear state estimation and attitude tracking. Nonlinear estimation techniques would be preferable [3], but the available, mature methodologies are linear. 


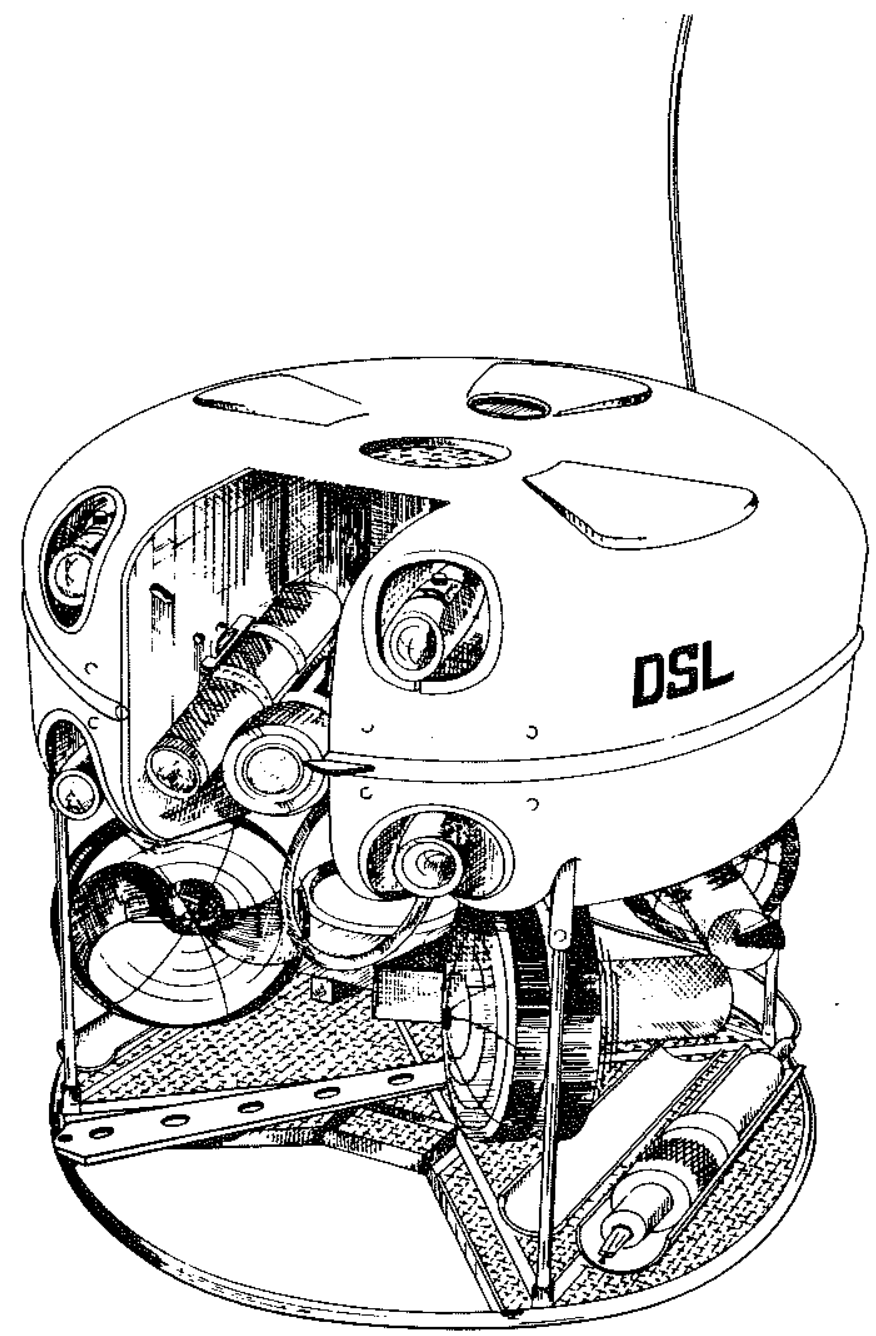

Figure 2-1: The Remotely Piloted Vehicle (RPV) used by Yoerger in his short range nonlinear closed loop control demonstrations. State measurements were made by a $1 \mathrm{MHZ}$ acoustic position measurement system. 


\subsection{Equations of Motion}

First we examine the equations of motion for an ROV. In this section we divide the examination of these equations into two parts. The first part is a detailed look at the equations of motion for a single degree of freedom. The second part extends the one dimensional case to three dimensions and presents the equations of motion for the full six degrees of freedom.

\subsubsection{Equations for a Single Degree of Freedom}

Newton's second law provides the starting point for the equations of motion. The second law states that the sum of the external forces is equal to the time rate of change of momentum of the vehicle. For a thruster propelled vehicle in a hydrodynamic environment, in one dimension this law becomes:

$$
F_{\text {thrusters }}-m_{\text {added }} \frac{d V(t)}{d t}=M_{\text {Inertial }} \frac{d V(t)}{d t}+C_{d r a g} \frac{1}{2} V(t)^{2} S
$$

This equation is really a parametric fit to the data collected thus far. It combines concepts from the theory of inviscid and viscous fluid flow. There are several limitations in this representation of the vehicle:

- The $M_{\text {added }}$ coefficient is actually time dependent in three dimensions. We have used a time-invariant approximation which is discussed below.

- $C_{d r a g}$ is also represented as a constant, when in fact it has been empirically shown to be a function of vehicle state.

- Other complicated phenomena which result when going from two dimensions to three,' such as vortex shedding, are not explicitly accounted for.

The following paragraphs discuss the use of the one dimensional description in more detail.

$M_{\text {added }}$ is the 'added mass coefficient' for motion in the direction of interest. The added mass effect, $m_{a d d e d} \frac{d V(t)}{d t}$, acts to inhibit acceleration or deceleration, while having no effect for steady motion relative to the fluid. The added mass coefficient is strictly dependent on the geometry of the vehicle. The choice of reference frame from which to view the motion is important, however. The force of an accelerating flow on a stationary body is greater 
than the force on a body with an equivalent acceleration through a stationary fluid. This last fact makes us treat body acceleration fluctuations and current fluctuations differently in calculating the resultant states. If viscous effects could be neglected, added mass would be the only external force opposing the motion of the ROV through the fluid.

$C_{d r a g}$ is the 'coefficient of drag' for the vehicle and the drag term, $C_{d r a g} \frac{1}{2} V(t)^{2} S$, is a measure of the force resisting steady and unsteady motions in the direction of interest. The coefficient of drag is a function of geometry and Reynolds number. Reynolds number itself is a function of a 'normalized' velocity of the body and is explained in [5] and [6]. Often used to represent the expected $C_{d r a g}$ for amorphous shapes such as JASON JUNIOR, the coefficient of drag has been experimentally measured over a large range of Reynolds numbers for a spherical body. The resulting plot of $C_{d r a g}$ versus Reynolds number is typically displayed in a log-log format. A plot of this curve from [5] is reproduced as figure (2-2).

While the drag curve for a sphere is nonlinear, several regions of distinctly different behavior can be discerned and related to specific hydrodynamic behavior. Using the ROV's JASON and JASON JUNIOR as a basis for calculating Reynolds number, we find that their operations are generally restricted to the left and central part of the curve. This could correspond to a fairly significant range of $C_{d r a g}$, however. For very small $\operatorname{Re} C_{d r a g}$ rises dramatically. Calculating an equivalent diameter based on the volume of JASON JUNIOR, for example, we can calculate $R e$ for a given speed. Surprisingly, If we consider two representative speeds, 0.01 and 1.0 knots, and calculate the corresponding $R e$, we find a very narrow spread in $C_{d r a g}$. For 1 knot:

$$
R e=\frac{U D}{\nu}=\frac{(.515 \mathrm{~m} / \mathrm{sec})(.5 \mathrm{~m})}{1.35 \times 10^{-6}}=1.9074 \times 10^{5} \Rightarrow 0.43
$$

For .01 knots:

$$
R e=\frac{U D}{\nu}=\frac{(.0052 \mathrm{~m} / \mathrm{sec})(.5 \mathrm{~m})}{1.35 \times 10^{-6}}=1.9074 \times 10^{3} \Rightarrow 0.44
$$

The reason for the narrow spread is that this portion of the curve is relatively flat. At approximately $1.6 \mathrm{Knots}$ (corresponding to $R e=3 \times 10^{5}$ ), the curve predicts a transition from laminar to turbulent flow and a large drop in $C_{d r a g}$ to a value of approximately 0.1 . Due to the additional discontinuities in the shape of JASON JUNIOR, however, the curve would be 


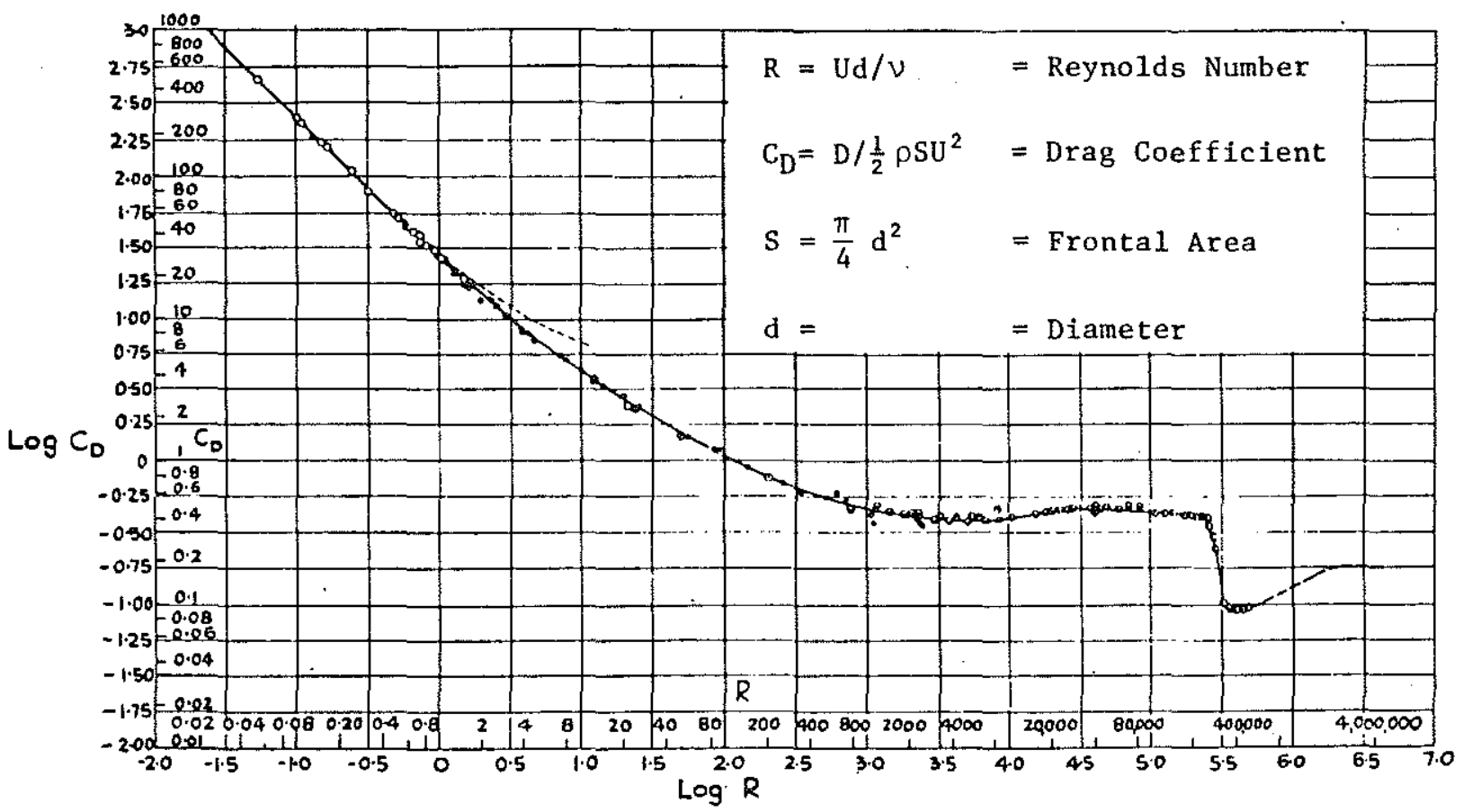

Figure 2-2: A log-log plot of $C_{d r a g}$ versus $R e$ for a submerged sphere. Taken originally from the 1938 Modern Developments in Fluid Dynamics by Goldstein.

expected to shift to the left because the transition to turbulent flow should occur at a lower speed.

Finally, the $S$ in the drag term represents the cross sectional area perpendicular to the direction of vehicle motion.

We can now make the equations of motion for the ROV more compact. By rearranging terms and combining $C_{d r a g} \frac{1}{2} S$ in a single coefficient, we get:

$$
F_{\text {thrusters }}=\left(M_{\text {inertial }}+m_{\text {added }}\right) \frac{d V(t)}{d t}+C_{d r a g}^{\prime} V(t)^{2}
$$

And rewriting the equations in State Space form:

$$
\begin{gathered}
\frac{d X(t)}{d t}=V(t) \\
\frac{d V(t)}{d t}=\frac{1}{\left(M_{\text {inertial }}+m_{\text {added }}\right)}\left(F_{\text {thrusters }}-C_{d r a g}^{\prime} V(t)^{2}\right)
\end{gathered}
$$




\subsubsection{Equations for Six Degrees of Freedom}

As expected, the extension to six degrees of freedom is complicated by coupling terms and includes complexities not encountered in the single degree of freedom. Moments resulting from the separation of the center of gravity from the center of buoyancy, for example, become important.

The six degrees of freedom are made up of three orthogonal directions of translational motion and three complimentary directions of rotational motion. Figure (2-3) illustrates these six degrees of freedom of motion.

Presented below are the equations of motion for the full six degree of freedom case. The basis for these equations is the set of equations developed for modern submarines by the David Taylor Naval Ship Research and Development Center (DTNSRDC) and are known as the Standard Submarine Equations of Motion [7].

Axial Force Equation:

$$
\begin{array}{r}
m\left[\dot{u}-v r+w q-x_{G}\left(q^{2}+r^{2}\right)+y_{g}(p q-\dot{r})+z_{g}(p r+\dot{q}]=\right. \\
\frac{\rho}{2} l^{4}\left[X_{q q^{\prime}} q^{2}+X_{r r^{\prime}} r^{2}+X_{r p^{\prime}}\right] \\
+\frac{\rho}{2} l^{3}\left[X_{\dot{u}^{\prime}}+X_{v r^{\prime}} v r+X_{w q^{\prime}}\right] \\
+\frac{\rho}{2} l^{2}\left[X_{v v^{\prime}} v^{2}+X_{w w^{\prime}} w^{2}\right] \\
+\frac{\rho}{2} l^{2}\left[X_{\delta r \delta r^{\prime}} u^{2} \delta_{r}^{2}+X_{\delta s \delta s^{\prime}} r^{2} u^{2} \delta_{s}^{2}+X_{\delta b \delta b^{\prime}} u^{2} \delta_{b^{2}}\right] \\
-(W-B) \sin (\theta)+\left(F_{\text {thrusters }}-\text { Drag }\right)
\end{array}
$$

Where: Drag $=C_{d r a g}^{\prime} u(t)^{2}$

Lateral Force Equation:

$$
m\left[\dot{v}-w p+u r-y_{G}\left(r^{2}+p^{2}\right)+z_{g}(q r-\dot{p})+X_{g}(q p+\dot{r}]=\right.
$$




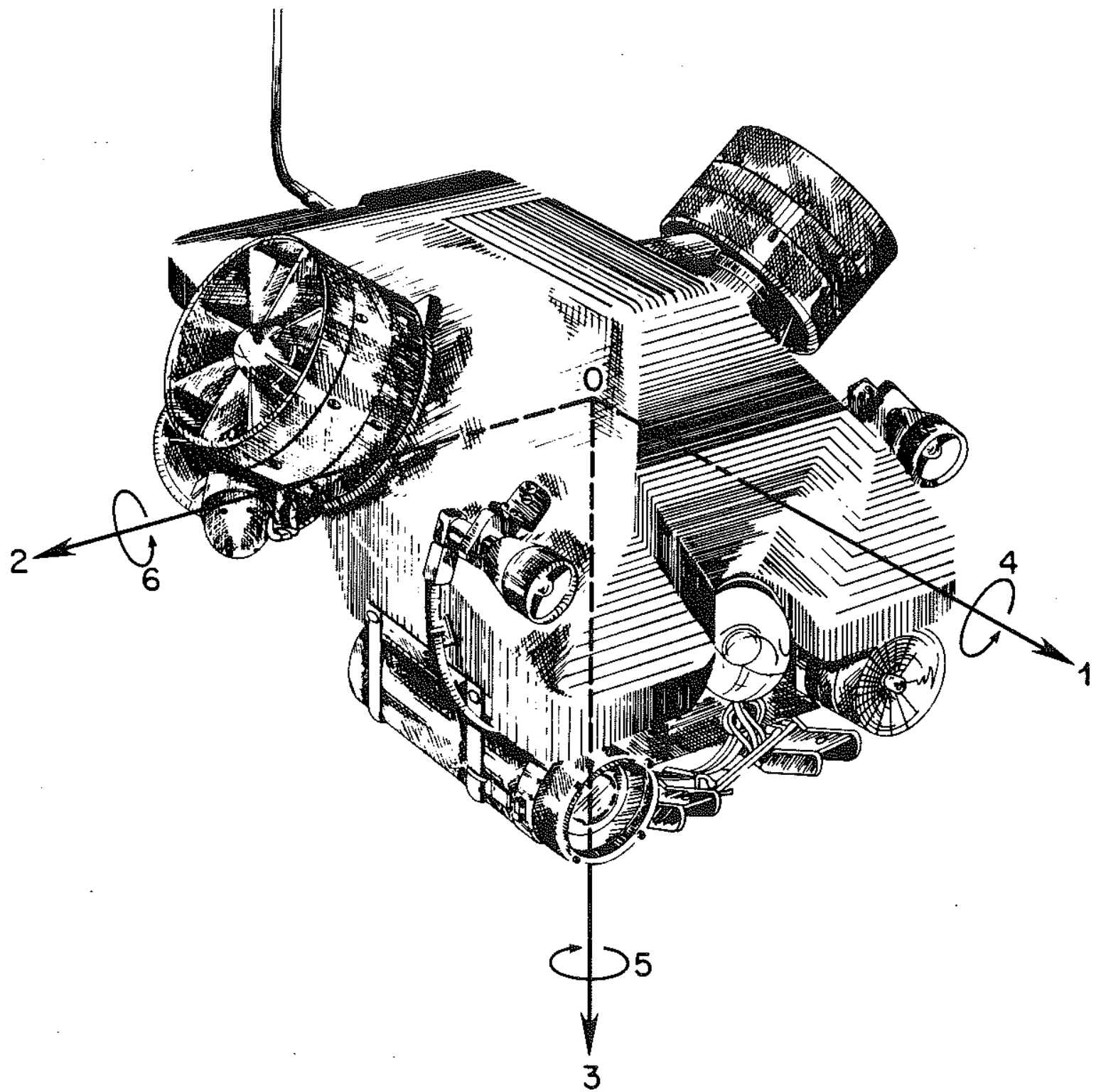

Figure 2-3: The six degrees of freedom for the ROV, JASON JUNIOR. Axes are chosen where possible to take advantage of vehicle symmetry. If the Z-axis (3) is chosen to be UP, the other axes must then be relabeled to satisfy the right hand rule convention. 


$$
\begin{array}{r}
\frac{\rho}{2} l^{4}\left[Y_{(r)^{\prime}} \dot{r}+Y_{\dot{p}^{\prime}} \dot{p}+Y_{p|p|} p|p|+Y_{p q^{\prime}} p q\right] \\
+\frac{\rho}{2} l^{3}\left[Y_{r^{\prime}} u r+Y_{p^{\prime}} u p+Y_{\dot{v}^{\prime}} \dot{v}+Y_{w p^{\prime}} w p\right] \\
+\frac{\rho}{2} l^{2}\left[Y_{\psi^{\prime}} u^{2}+Y_{v^{\prime}} u v+Y_{v|v| R^{\prime}} v\left|\left(v^{2}+w^{2}\right)^{\frac{1}{2}}\right|\right] \\
+\frac{\rho}{2} l^{2}\left[Y_{\delta r^{\prime}} u^{2} \delta_{r}+Y_{\delta r \eta^{\prime}} u^{2} \delta_{r}\left(\eta-\frac{1}{C}\right) C\right] \\
-\frac{\rho}{2} C_{d} \int_{l} h(x) v(x)\left\{[w(x)]^{2}+[v(x)]^{2}\right\}^{\frac{1}{2}} d x \\
-\frac{\rho}{2} l \bar{C}_{L} \int_{x_{2}}^{x_{1}} w(x) v_{F W}(t-\tau(x)) d x \\
+(W-B) \cos \theta \sin \phi
\end{array}
$$

Vertical Force Equation:

$$
\begin{array}{r}
m\left[\dot{w}-u q+v p-z_{G}(r p-\dot{q})+y_{G}(r q+\dot{p})\right]= \\
\frac{\rho}{2} l^{4} Z_{\dot{q}^{\prime}} \dot{q} \\
+\frac{\rho}{2} l^{3}\left[Z_{\dot{w}^{\prime}} \dot{w}+Z_{q^{\prime}} u q+Z_{v p^{\prime}} v p\right] \\
\frac{\rho}{2} l^{2}\left[Z_{*^{\prime}} u^{2}+Z_{w^{\prime}} u w\right] \\
\frac{\rho}{2} l^{2}\left[Z_{|w|^{\prime}} u|w|+Z_{w w^{\prime}}\left|w\left(v^{2}+w^{2}\right)^{\frac{1}{2}}\right|\right] \\
+\frac{\rho}{2} l^{2}\left[Z_{\delta s^{\prime}} u^{2} \delta_{s}+Z_{\delta s \eta^{\prime}} u^{2} \delta_{s}\left(\eta-\frac{1}{C}\right) C\right] \\
-\frac{\rho}{2} C_{d} \int_{l} b(x) w(x)\left\{[w(x)]^{2}+[v(x)]^{2}\right\}^{\frac{1}{2}} d x \\
-\frac{\rho}{2} l \bar{C}_{L} \int_{x_{2}}^{x_{1}} v(x) v_{F W}(t-\tau[x]) d x \\
+(W-B) \cos \theta \sin \phi
\end{array}
$$

Rolling moment equation:

$$
\begin{gathered}
I_{x} \dot{p}+\left(I_{z}-I_{y}\right) q r-(\dot{r}+p \dot{q}) I_{z x}+(p r-\dot{q}) I_{x y} \\
+m\left[y_{G}(\dot{w}-u q+v p)-z_{G}(\dot{v}-w p+u r)\right]= \\
\frac{\rho}{2} l^{5}\left[K_{\dot{p}^{\prime}} \dot{p}+K_{\dot{r}^{\prime}} \dot{r}+K_{q r^{\prime}} q r+K_{p|p|^{\prime}} p|p|\right] \\
+\frac{\rho}{2} l^{4}\left[K_{p^{\prime}} u p+K_{r^{\prime}} u r+K_{\dot{v}^{\prime}} \dot{v}+K_{w p^{\prime}} w p\right] \\
+\frac{\rho}{2} l^{3}\left[K_{*^{\prime}} u^{2}+K_{v R^{\prime}} u v+K_{1^{\prime}} u v_{F W}\left(t-\tau_{T}\right)\right] \\
+\frac{\rho}{2} l^{3}\left[K_{\delta r^{\prime}} u^{2} \delta_{r}+K_{\delta r \eta^{\prime}} u^{2} \delta_{r}\left(\eta-\frac{1}{C}\right) C\right] \\
+\left(y_{G} W-y_{B} B\right) \cos \theta \cos \phi-\left(z_{G} W-z_{B} B\right) \cos \theta \sin \phi-Q_{p}
\end{gathered}
$$


Pitching moment equation:

$$
\begin{array}{r}
I_{y} \dot{q}+\left(I_{x}-I_{z}\right) r p-(\dot{p}+q r) I_{x y}+\left(p^{2}-r^{2}\right) I_{z x}+(q p-\dot{r}) I_{y z} \\
+m\left[z_{G}(\dot{u}-v r+w q)-x_{G}(\dot{w}-w q+v p)\right]= \\
\frac{\rho}{2} l^{5}\left[M_{\dot{q}^{\prime}} \dot{q}+M_{r p^{\prime}} r p\right] \\
+\frac{\rho}{2} l^{4}\left[M_{\dot{w}^{\prime}} \dot{w}+M_{q^{\prime}} u q\right] \\
+\frac{\rho}{2} l^{3}\left[M_{\psi^{\prime}} u^{2}+M_{w^{\prime}} u w+M_{w|w| R^{\prime}} w\left|\left(v^{2}+w^{2}\right)^{\frac{1}{2}}\right|\right] \\
+\frac{\rho}{2} l^{3}\left[M_{|w|^{\prime}} u|w|+M_{w w^{\prime}}\left|w\left(v^{2}+w^{2}\right)^{\frac{1}{2}}\right|\right] \\
+\frac{\rho}{2} l^{3}\left[M_{\delta s^{\prime}} u^{2} \delta_{s}+M_{\delta b^{\prime}} u^{2} \delta b+M_{\delta s \eta^{\prime}} u^{2} \delta_{s}\left(\eta-\frac{1}{C}\right) C\right] \\
+\frac{\rho}{2} C_{d} \int_{l} x b(x) w(x)\left\{[w(x)]^{2}+[v(x)]^{2}\right\}^{\frac{1}{2}} d x \\
-\frac{\rho}{2} l \bar{C}_{L} \int_{x_{2}}^{x_{1}} x v(x) v_{F W}(t-\tau[x]) d x \\
-\left(x_{G} W-x_{B} B\right) \cos \theta \cos \phi-\left(z_{G} W-z_{B} B\right) \sin \theta
\end{array}
$$

Yawing moment equation:

$$
\begin{array}{r}
I_{z} \dot{r}+\left(I_{y}-I_{x}\right) p q-(\dot{q}+r p) I_{y z}+\left(q^{2}-p^{2}\right) I_{x y}+(r q-\dot{p}) I_{x y} \\
+m\left[x_{G}(\dot{v}-w p+u r)-y_{G}(\dot{u}-v r+w q)\right]= \\
\frac{\rho}{2} l^{5}\left[N_{\dot{r}^{\prime}} \dot{r}+N_{\dot{p}^{\prime}} \dot{p}+N_{p q^{\prime}} p q\right] \\
+\frac{\rho}{2} l^{4}\left[N_{p^{\prime}} u p+N_{r^{\prime}} u r+N_{\dot{v}^{\prime}} \dot{v}\right] \\
\left.+\frac{\rho}{2} l^{3}\left[N_{*^{\prime}} u^{2}+N_{v^{\prime} u v} u N_{v|v| R^{\prime}} v \mid\left(v^{2}+w^{2}\right)^{\frac{1}{2}}\right]\right] \\
+\frac{\rho}{2} l^{3}\left[N_{\delta r^{\prime}} u^{2} \delta_{r}+N_{\delta \eta^{\prime}} u^{2} \delta_{r}\left(\eta-\frac{1}{C}\right) C\right] \\
-\frac{\rho}{2} C_{d} \int_{l} x h(x) v(x)\left\{[w(x)]^{2}+[v(x)]^{2}\right\}^{\frac{1}{2}} d x \\
-\frac{\rho}{2} l \bar{C}_{L} \int_{x_{2}}^{x_{1}} x v(x) v_{F^{\prime} W}(t-\tau[x]) d x \\
+\left(x_{G} W-x_{B} B\right) \cos \theta \sin \phi+\left(y_{G} W-y_{B} B\right) \sin \theta
\end{array}
$$

Again limitations restrict the indiscriminate use of these equations. For the ROV's JASON and JASON JUNIOR, the following considerations are important:

- The propulsion and methods of maneuvering for ROV's are significantly different.

- Speed regimes for ROV's are typically much slower and result in significantly different dynamics. Depending on the environment and the mission, static concerns can predominate over dynamic ones for the ROV. 
- ROV Appendages such as manipulators, tethers, and various equipment installed for specific tasks, are not addressed.

It should be pointed out that the actual hydrodynamic characteristics or coefficients of such a complicated structure as JASON or JASON JUNIOR have not been determined. The equations presented represent a best guess of the dynamics of the vehicles using our understanding of the hydrodynamic drag and added mass and do not specifically address such issues as tether drag. Clearly there is a great deal of uncertainty in the coefficients presented and for the speed regimes in which the two vehicles operate.

The equations developed by DTNSRDC are specifically tailored for the case of a stern propelled submarine with control surfaces. JASON JUNIOR and JASON do not have control surfaces, however. These ROV's accomplish motion control with the use of multiple, independently controllable, thrusters. JASON JUNIOR has a total of four thrusters, while JASON has a total of seven.

\subsection{Dynamic Distinctions between JASON and JASON JU- NIOR}

While both vehicles are conceptually similar, the implementation details and mission capability of JASON and JASON JUNIOR are considerably different. These differences manifest themselves in different dynamic characteristics.

\subsubsection{Dynamics of JASON JUNIOR}

As mentioned above there are a total of four thrusters on JASON JUNIOR. There are two stern thrusters pointing forward, with opposite cants of 30 degrees from straight ahead. There are two more thrusters pointing up, canted toward each other with cants of about 30 degrees from straight up. Figure (2-4) is an illustration of JASON JUNIOR which highlights its major features.

Comparing the illustration of JASON JUNIOR with that of the RPV it is clear that JASON JUNIOR has been streamlined for forward motion. The horizontal thrusters are positioned for maximum effectiveness in the forward direction, the syntactic foam body is designed with forward motion in mind, and the tether is attached to the very tail of the vehicle 

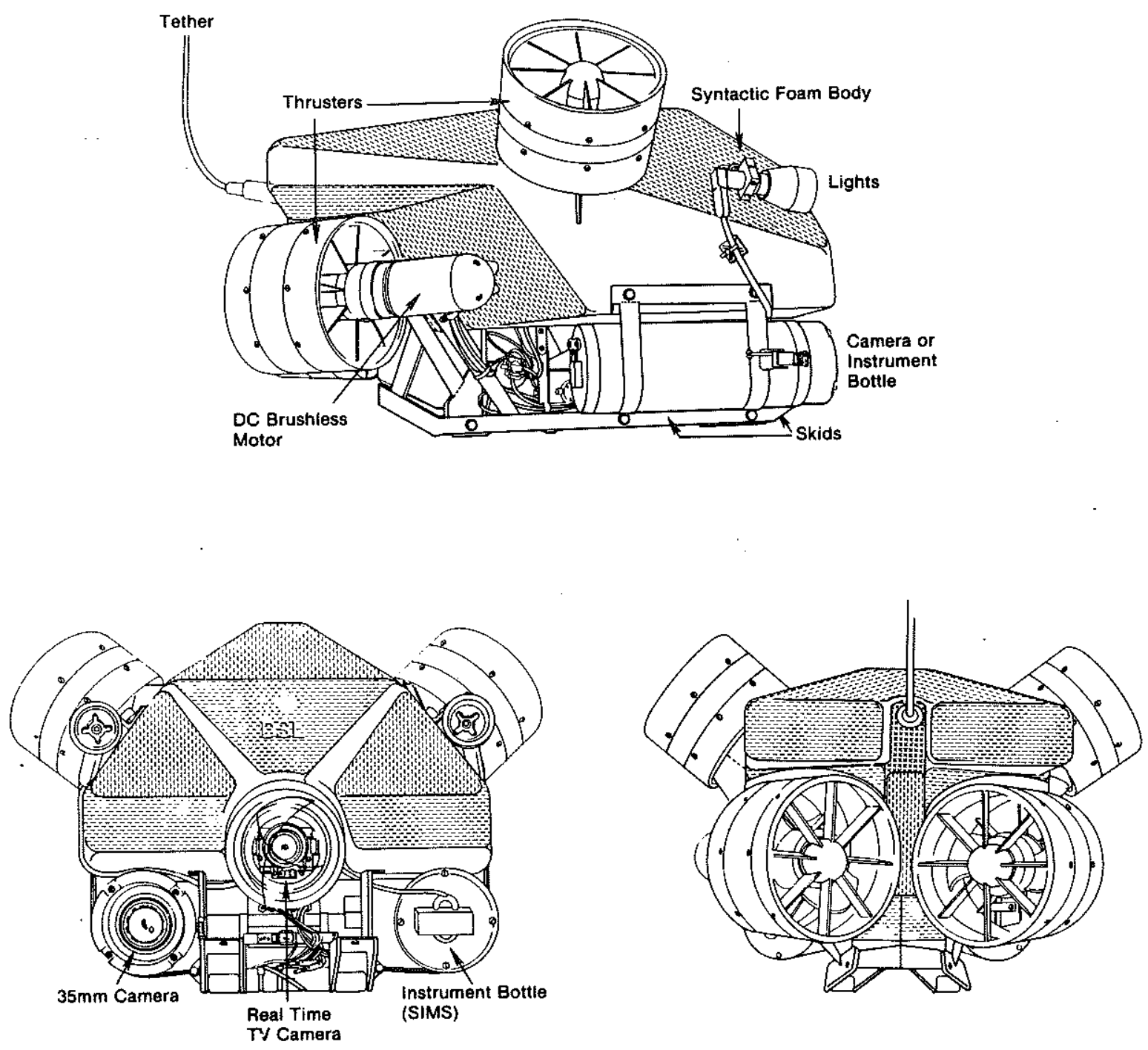

Figure 2-4: Drawings of JASON JUNIOR in its actual configuration for pool testing of the hybrid state estimator. For this testing the $35 \mathrm{~mm}$ still camera in the port instrument area has been replaced with the attitude package described in Chapter 4. Eventually, the starboard instrument port was used for the enclosure of a pressure depth sensor housed in an initial design of the attitude package. 
to allow maximum maneuverability in forward travel. Changes of direction are accomplished by applying twisting thrust via the two lower thrusters.

Designed for deployment from the manned submersible ALVIN in the 1986 exploration of the Titanic, its forward pointing TV, still camera and lights, explain why streamlining for forward travel was desired. Figure (2-5) illustrates the operational scenario involved in the 1986 deep submergence expedition.

Because of the location of the horizontal thrusters relative to the center of gravity and buoyancy, forward travel causes the bow of the vehicle to pitch upward. As expected, the opposite effect results under reverse thrust. The natural frequency of this potential oscillation is approximately $.33 \mathrm{HZ}$. Similar moments are created during the application of lateral thrusts. This potential oscillation has a natural frequency of about .5 HZ.

\subsubsection{JASON Dynamics}

Although JASON is also a tethered vehicle with independently controllable thrusters for motion control, it was designed with a more ambitious list of possible mission objectives. Among other things, JASON

1. occupies nearly twelve times the volume that JASON JUNIOR occupies,

2. is nearly a parallelpiped, and

3. will have a manipulator mounted on its lower forward starboard corner.

A three dimensional view of JASON is included in this section as Figure (2-6).

The manipulator alone, had a significant affect on the design and hence the dynamics of the vehicle. In order to make the manipulator useful, JASON's thrusters had to be significantly more powerful than those on JASON JUNIOR. The design objective was to lift a payload of $30 \mathrm{lbs}$ to the surface, maintaining a level attitude on the vehicle. JASON's thrusters are nearly symmetrical and have sufficient separation so that no cants off the vehicle's axes are required. (Figure (2-6) shows some cant to the thrusters that was anticipated in the initial drawings, but not implemented.) JASON's design thrust objectives were achieved by:

- more thrusters (seven total), and 


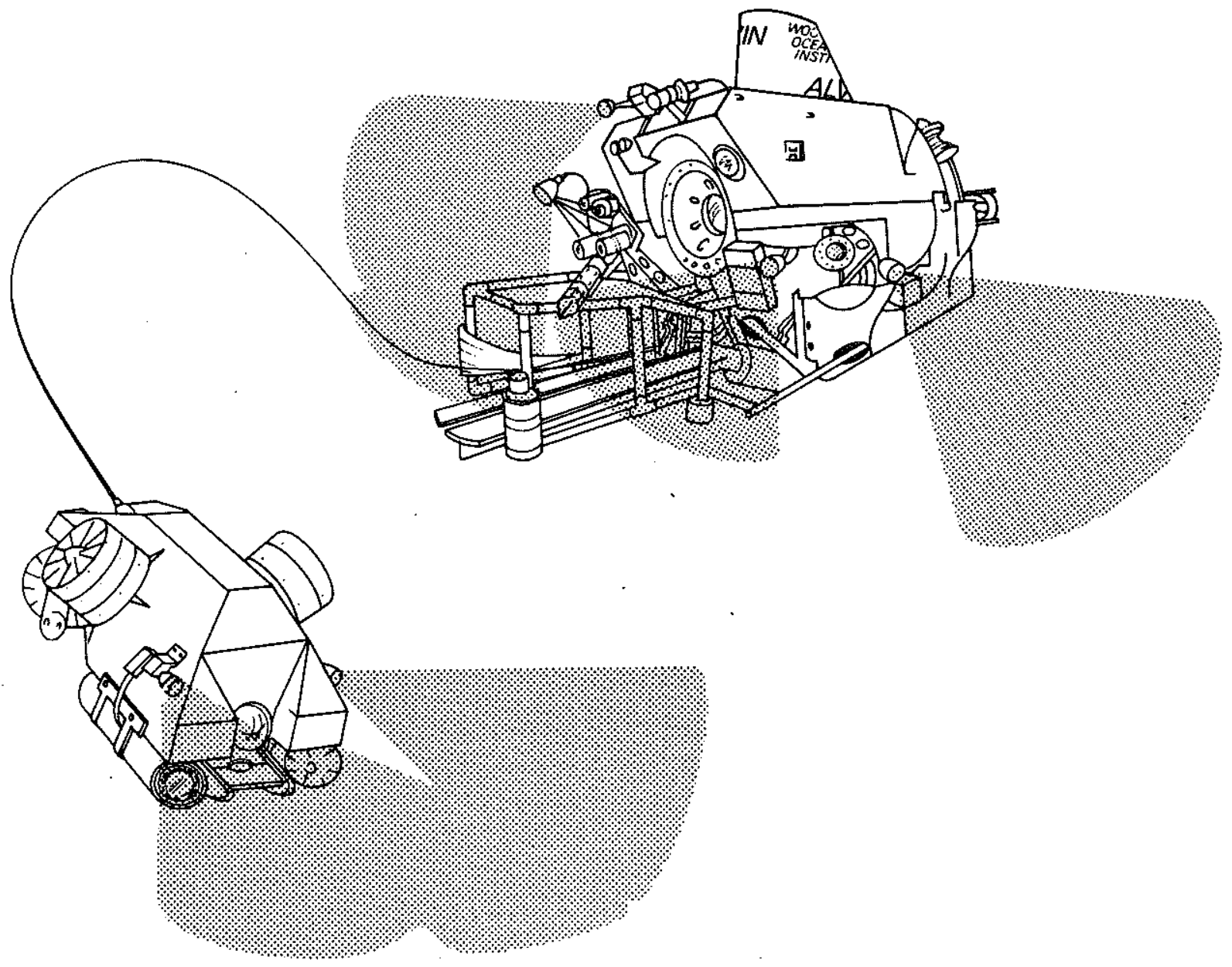

Figure 2-5: An illustration of the operational scenario involving the deployment of JASON JUNIOR during the 1986 Exploration of the RMS Titanic. This scenario was one of the driving forces in the design of the ROV. 


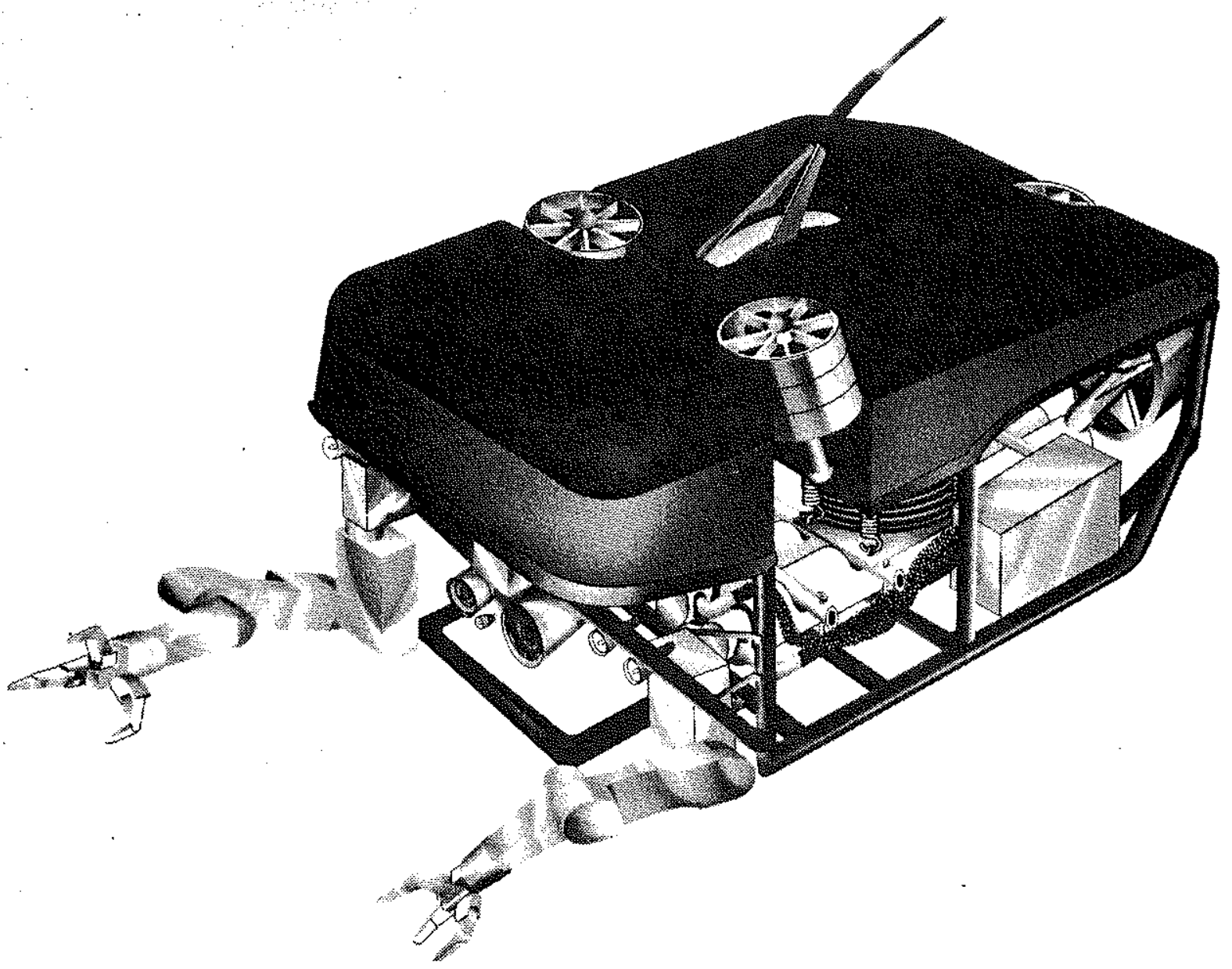

Figure 2-6: A drawing of ROV JASON in its final projected configuration. Currently undergoing pool and dockside testing, JASON is scheduled for its maiden mission in October of 1988. One manipulator is currently undergoing separate testing. Integrated manipulator-vehicle testing is scheduled for a later date. 
- more hydrodynamically efficient ducting of the thrusters.

Although not quite as hydrodynamically biased toward forward travel as JASON JUNIOR, JASON has its manipulator, cameras and lights all pointing forward. The operator's objectives still clearly center on positioning the 'bow' of the vehicle. 


\section{Chapter 3}

\section{Discrete Linear Observers in Underwater Vehicle Control}

This chapter explains the full state feedback approach to closed loop control and examines the role that observers can play in facilitating a form of full state feedback using state estimates. Depending on the estimation process dynamics, the estimates may result in lower noise levels than actual measurements and/or a higher control bandwidth.

\subsection{Closed Loop Versus Open Loop Control}

If the mathematical model developed for an ROV were perfect and there were no unforseen disturbances encountered during flight, an ROV could be controlled with a control system which is simply the inverse of the plant model. There would be no need to monitor vehicle states. This strategy is the basic assumption in Open Loop control. In reality, however, modeling uncertainty and unknown external disturbances prevent all models from being exact descriptions of physical systems.

This uncertainty in modeling is particularly true for an ROV like JASON or JASON JUNIOR, whose hydrodynamic properties are not only not precisely calculated, but whose properties will change due to manipulator action, tether dynamics, and current fluctuations. Because of these uncertainties, the resulting vehicle states must be monitored and used to correct the control signals to achieve the desired state or state trajectory. This monitoring is commonly known as feedback. We call the combination of monitoring and correction Closed Loop Control. 
Open Loop (State Space)

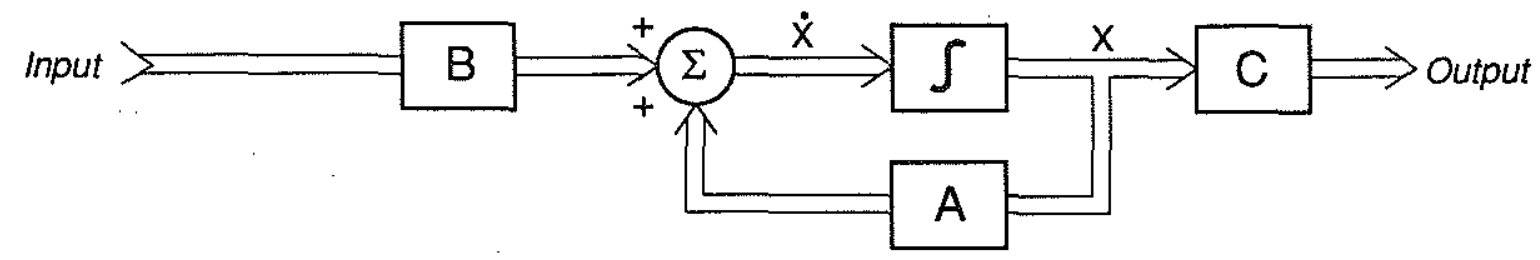

Closed Loop (State Space)

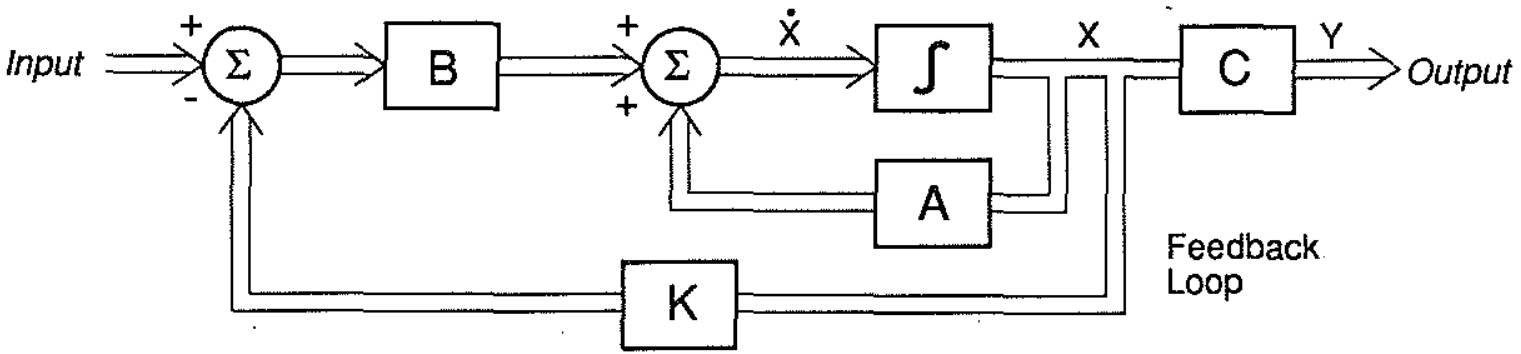

Figure 3-1: Continuous time domain representations of open and closed loop systems. These block diagrams use standard state space notation.

Most modern systems employ some type of analog or digital feedback to close the loop. In fact, many 'open loop' control systems could be considered closed loop systems because the operator is used to make the measurements and the necessary control corrections. This latter description is the method in which JASON JUNIOR was used in the exploration of the RMS Titanic; the operator used real time TV to guide the ROV. The system classification depends on where we draw the boundary between the 'system' and the 'environment'. For the purposes of this investigation, the operator is in the environment.

Simple block diagrams of an open loop and a closed loop control system are illustrated in Figure (3-1). Block diagrams are not unique representations of the system and can be drawn in a number of different forms. The block diagrams of Figure (3-1) are drawn in a manner typical of time domain/state space representations. 


\subsection{Full State Feedback and Selection of ROV Dynamics}

Figure (3-1) illustrated a closed loop system with a gain block in the feedback line. If the gain block passes the feedback without alteration, then the system is said to have unity feedback. But rather than simply passing the output back to the input, we can tailor the feedback by selecting specific values to fill the feedback gain matrix. This section presents the methodology for filling that matrix in the context of ROV control.

\subsubsection{Full State Feedback Structure}

For ROV's, full state feedback in continuous time refers to the continuous monitoring of position and velocity and the continuous correction to thruster commands. The following equations represent Full State Feedback for a linearization of the equations developed for a single degree of freedom in Chapter 2.

$$
\begin{aligned}
\dot{x} & =\bar{A} \vec{x}+\bar{B} u \\
y & =\bar{C} \vec{x}+\bar{D} u \\
u & =-\bar{K} \vec{x}
\end{aligned}
$$

where:

$$
\begin{gathered}
A=\left[\begin{array}{cc}
0 & 1 \\
0 & \frac{-\beta_{\text {drap }}}{M_{\text {inertial }}+m_{\text {added }}}
\end{array}\right] \quad B=\left[\begin{array}{c}
0 \\
\frac{1}{M_{\text {inertial }}+m_{\text {added }}}
\end{array}\right] \\
C=\left[\begin{array}{ll}
1 & 0
\end{array}\right] \quad D=\left[\begin{array}{l}
0
\end{array}\right]
\end{gathered}
$$

\subsection{Discrete Implementation of Full State Feedback}

Contrary to the assumption of continuous time system descriptions, the attitude and position measurement systems implemented on JASON JUNIOR do not provide continuous measurements or feedback. Information from these systems is sampled at discrete intervals of time. As well, the algorithms which compute the thruster commands are implemented in a digital computer, generating corrections at discrete intervals. The implementation, then, drives us to a discrete development of full state feedback. 


\subsubsection{Transformation to Discrete Time Representation}

Although the equations developed above are continuous time expressions of vehicle dynamics, several methods are available to discretize the continuous descriptions for use with a digital computer. One method is to convert the A and B matrices of the continuous state space description to discrete Phi $(\Phi)$ and Gamma $(\Gamma)$ matrices. There are several ways to accomplish this conversion. Conversion options are discussed in [8],[9], and [10]. We will use the first three terms of the series expansion of the matrix exponential to convert to discrete time. The discrete equivalent of the single degree of freedom state equations are presented below in matrix form:

$$
\left[\begin{array}{c}
x_{k+1} \\
\dot{x}_{k+1}
\end{array}\right]=\left[\begin{array}{cc}
\Phi_{11} & \Phi_{12} \\
\Phi_{21} & \Phi_{22}
\end{array}\right]\left[\begin{array}{c}
x_{k} \\
\dot{x}_{k}
\end{array}\right]+\left[\begin{array}{c}
\Gamma_{11} \\
\Gamma_{21}
\end{array}\right] u(k)
$$

Where:

$$
\begin{aligned}
& \Phi_{11}=1 \\
& \Phi_{12}=T\left(1-\frac{T}{2}\left(\frac{\beta_{d r a g}}{M_{\text {inertial }}+m_{\text {added }}}\right)+\frac{T^{2}}{6}\left(\frac{\beta_{\text {drag }}}{M_{\text {inertial }}+m_{\text {added }}}\right)^{2}\right) \\
& \Phi_{21}=0 \\
& \Phi_{22}=1-T\left(\frac{\beta_{d r a g}}{M_{\text {inertial }}+m_{\text {added }}}\right)-\frac{T^{2}}{2}\left(\frac{\beta_{\text {drag }}}{M_{\text {inertial }}+m_{\text {added }}}\right)^{2}-\frac{T^{3}}{6}\left(\frac{\beta_{d r a g}}{M_{\text {inertial }}+m_{\text {added }}}\right)^{3} \\
& \Gamma_{11}=\frac{T^{2}}{2\left(M_{\text {inertial }}+m_{\text {added }}\right)}\left(1-\frac{T}{3}\left(\frac{\beta_{\text {drag }}}{M_{\text {inertial }}+m_{\text {added }}}\right)\right) \\
& \Gamma_{21}=\frac{T}{\left(M_{\text {inertial }}+m_{\text {added }}\right)}\left(1-\frac{T}{2}\left(\frac{\beta_{\text {drag }}}{M_{\text {inertial }}+m_{\text {added }}}\right)-\frac{T^{2}}{6}\left(\frac{\beta_{\text {drag }}}{M_{\text {inertial }}+m_{\text {added }}}\right)^{2}\right)
\end{aligned}
$$

For practical reasons, the discrete time conversions are generally done numerically and not symbolically. Computer simulation packages, such as PC MATLAB are often used for the calculations when numbers are available for the matrix elements. (The PC MATLAB conversion uses a matrix exponential which is calculated by the Pade Approximation.)

In the above equations $\mathrm{T}$ represents the sampling period. As can be seen from the structure, changes to the sampling period have a significant effect on the dynamics of the system. 
Proper selection of the sampling rate is not a trivial task and requires the consideration of numerous factors.

\subsubsection{Sampling Rate Selection}

Theoretically, sampling rates must be twice the frequency of the signal of interest. This is the Nyquist criteria and applies in a post processing sense when all past and present samples are available for use in the reconstruction of the original signal. In the context of 'real time' control this acausal algorithm is of little use. In actual practice a sampling frequency four to twenty times the frequency of interest is recommended, depending on the author ([8],[9]). A practical reason that authors quote such a large range is that measured signals can often contain much higher frequencies than those anticipated. It would also be fair to say that the frequency content and its variability are a function of the quantity being measured, the sensor/circuitry you are using to measure it, and the environment in which the quantity is being measured.

Sampling rate may be determined by computational limits, limits in telemetry, or sensor limitations. With JASON JUNIOR, the maximum sampling rate is currently fixed by the frequency of data transmission from the attitude package and the maximum update rate available from the SHARPS system.

Hardware constraints in the attitude package limit sensor sampling rate to approximately $10 \mathrm{HZ}$. The sensors in the package have analog outputs which are sampled by a single 12 bit $\mathrm{A}$ to $\mathrm{D}$ converter. The digital information is then transmitted serially to the surface computer. The attitude package is discussed in more detail in Chapter 4.

SHARPS update limits are not as clear cut. As mentioned previously, at long range, the SHARPS update rate is an inverse function of the acoustic wave travel time or reverberation time, whichever is longer. At short range, the SHARPS update rate is limited by the combination of travel time, processing time and environmental considerations. Which factor is more important in short range applications depends on the individual circumstances.

Examples of environmental considerations which may affect SHARPS update rate are the reverberation limitations associated with enclosure boundaries (such as pool, tank, or canyon walls) or large objects (such as ship hulls) which are in close proximity to the ROV. In general, 
the reverberation limitation becomes more severe as the frequency of the acoustic positioning system is lowered. In actual testing at $300 \mathrm{KHZ}$ in the large steel tank of the Coastal Research Laboratory, Woods Hole, reliable SHARPS update rates were limited to approximately $5 \mathrm{HZ}$. Measures can be taken to limit the reverberations, but ultimately the reverberation prone environment results in degraded performance. Possible corrective measures include reducing transducer output power, increasing the acoustic frequency, or adding acoustic insulation to the boundaries, or range-gating.

\subsection{Limitations of Full State Feedback}

In a linear, continuous, noise-free system (with full state feedback available) we can arbitrarily place the poles of our system anywhere in the S-plane. Implementation issues often restrict our freedom in selecting a system's closed loop dynamics, however.

\subsubsection{Low Update Rates}

In a discrete implementation the system cannot operate any faster than the update rate of the state measurements. Since update or sampling rate is explicitly used in the calculation of the $\Phi$ and $\Gamma$ matrices, this restriction has a significant impact. In general, lower update rates will translate into slower response to unpredicted state changes and therefore into larger changes between successive actuator signals. The net result of lower update rates is a smaller control bandwidth. Simulated closed loop performance for four different sampling rates were presented in Figure (1-1).

For the simulation pictured, the input was a step change in position. In order to pro duce realistic results the control model purposely has a different description than the actual state generator in the simulation loop. The control model is utilizing a linearized dynamic model, while the state generator is using a 4 th order Runge-Kutta approximation to the $C_{\text {drag }}^{\prime} V(t)|V(t)|$ expression for the hydrodynamic drag term.

\subsubsection{Noisy or missing measurements}

Noise-free measurements are assumed in the development of full state feedback. To some extent noise is always present in measurements, however. Its effect is to misrepresent state 


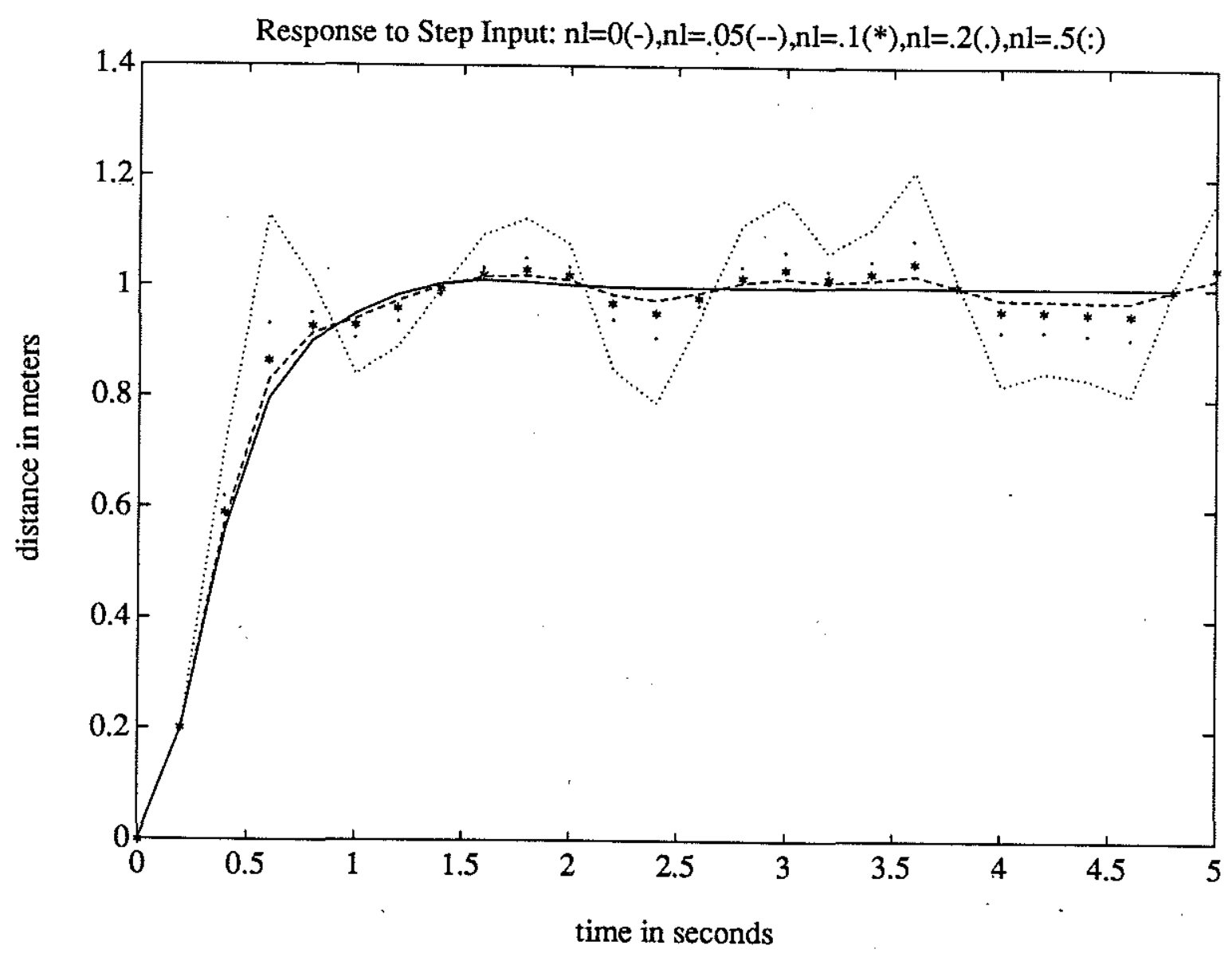

Figure 3-2: Simulated system performance with progressively higher sensor noise levels.

values. This misrepresentation causes incorrect adjustments to actuator control signals which results in a degradation of system performance (in much the same way that inaccurate modeling does.) The larger the noise level, the more detrimental the effect. Figure (3-2) illustrates the progressive deterioration of system performance with increasingly noisy measurements.

The effect of the noisy measurements is also apparent in looking at the simulated actuator demands generated by the control system when the noise in the measurement is varied. Figure (3-3) is the companion graph of control actions corresponding to the position plots of Figure (3-2).

Clearly, the combination of both noisy sensors and low update rates is very damaging to efforts to raise the performance level of closed loop control of an ROV. 


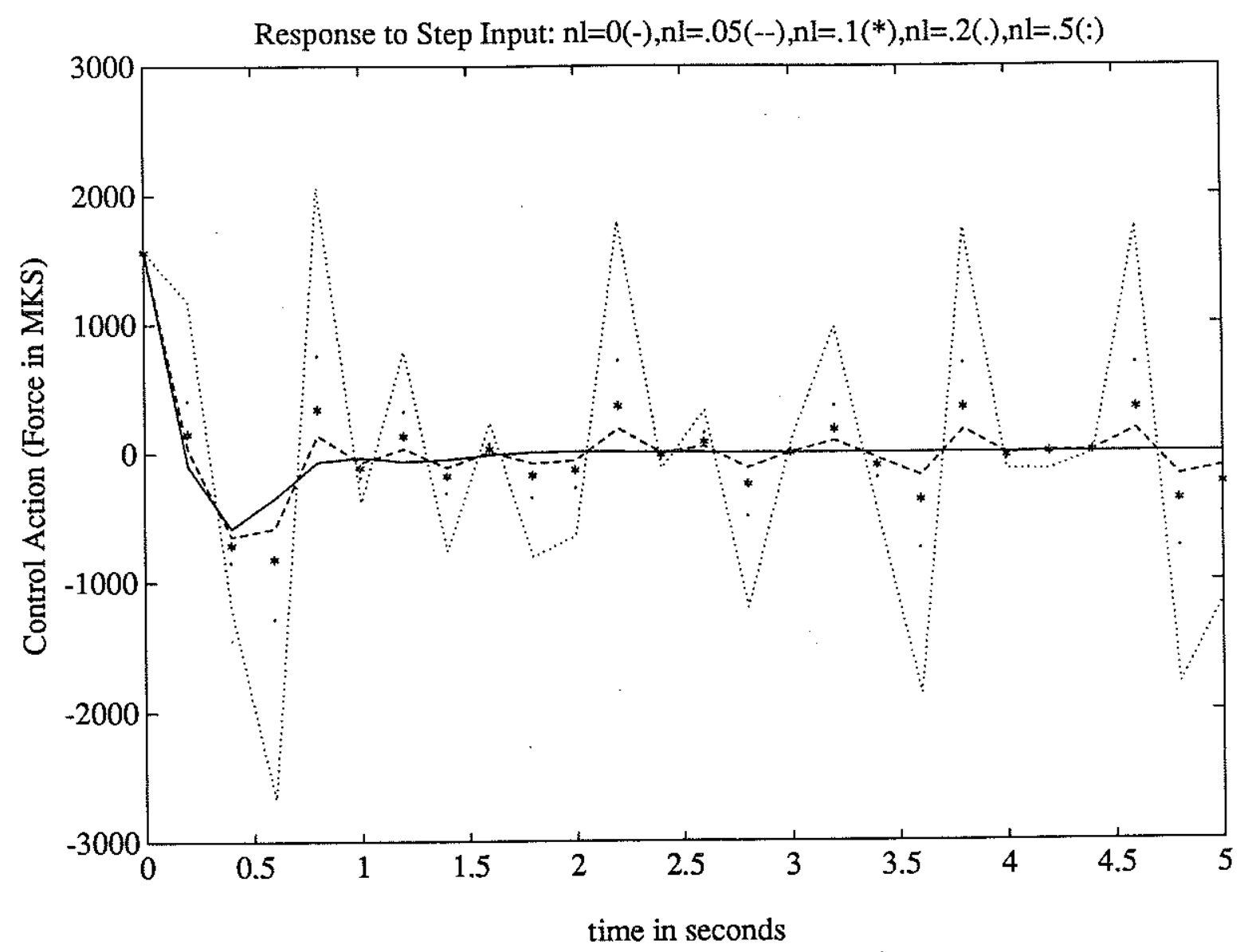

Figure 3-3: Simulated system performance and corresponding actuator commands for five different noise levels. Significant degradation in performance can occur when control commands saturate the actuator. Peakiness in the control commands can also exceed the limits of actuator dynamic response.

\subsubsection{Steady State Error}

Even if the state measurements are noise-free there is no guarantee that there will be no steady-state error. It is still essentially a P-D controller. System type will still determine the nature of the steady state error. The system used to describe JASON JUNIOR is known as a type I system. This means that the continuous time, open loop transfer function has a free integrator in the denominator. As a result, given a step change in the desired position for the ROV, the actual steady state position should be identical to that ordered. For a ramp input we predict a constant steady state error. For a parabolic input or higher order input, 
the error will grow without bound.

In order to eliminate error to a ramp input, integral action would be required. This is not generally desirable for our applications.

\subsection{Full Order Observers}

If measurement noise is significantly affecting closed loop system performance, it may be possible to reduce the noise level through the use of an observer. The observer in this case would behave as a filter, providing state estimates with, hopefully, less noise. Likewise, if all of the states are not measured, it may be possible to estimate them so that the effect of full state feedback can be recovered even without all of the measurements.

One approach used for missing measurements is to build an observer which uses an identical model of the vehicle as the control system does. Since the output of this type of observer contains all of the states it is classified as a full order observer. Because it shares the same model as the control system, this particular form is called an identity observer [11].

There are other observer structures in addition to the identity observer. For example, a reduced order observer is one which only feeds back estimates of a subset of the system states [12]. The remaining states are those that are actually measured and have acceptable noise characteristics.

\subsubsection{Discrete-Time Observer Structure}

A block diagram of a discrete, full order, identity observer is presented as figure (3-4).

As mentioned in the overview, the states estimated by the observer are fed back to the overall system. The error between the measurement(s) and the observer output is passed through the observer gain matrix and fed back to the input of the Observer.

\subsubsection{Observability}

A full-order observer cannot be applied indiscriminately. The system must be of such a structure that knowing the past inputs and outputs we can determine its initial states. Although the mathematical statement of this theorem is slightly different for the discrete and continuous cases, the structural property is the same. We say that the system must be 


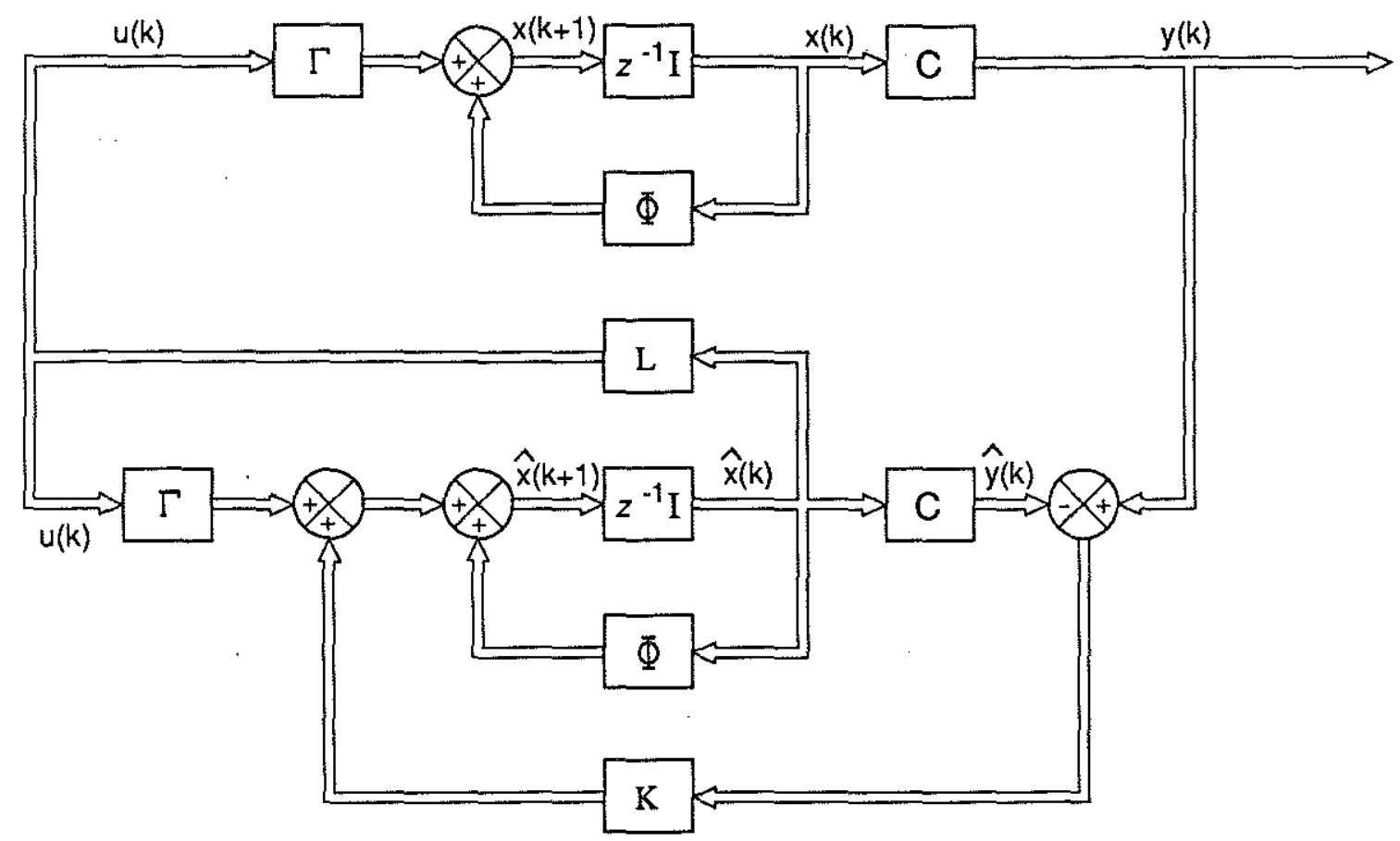

Figure 3-4: Block diagram of a discrete time, full order, linear, identity observer with state space notation.

Observable.

A system is observable if and only if the rank of its observability matrix is equal to the order of the system. Equation (3.6) shows the construction of the discrete observability matrix.

$$
W_{o}=\left[\begin{array}{l}
C \\
C \Phi \\
C \Phi^{2} \\
\vdots \\
C \Phi^{n-1}
\end{array}\right]
$$

The linear models of both JASON and JASON JUNIOR pass the observability test when position is measured. 


\subsubsection{Observer and Error Dynamics}

Once the system is shown to be observable, the dynamics that are desired for the observer must be selected. After the desired dynamics are selected, feedback gains for the Observer can be determined by pole placement techniques. These techniques are the same as those used in the full state feedback case. In this case, the observer gain matrix elements are the variables which allow us to pick arbitrary pole locations. The following equations show how the observer gain matrix influences the closed loop $\Phi$ matrix.

Expression for just the observer loop:

$$
\hat{x}_{k+1}=\Phi \hat{x}_{k}+\Gamma u_{k}+K\left[y_{k}-C \hat{x}_{k}\right]
$$

where:

$$
\begin{gathered}
y_{k}=C x_{k} \\
u_{k}=-L \hat{x}_{k}
\end{gathered}
$$

After combining terms:

$$
\hat{x}_{k+1}=[\Phi-K C-\Gamma L] \hat{x}_{k}+K C x_{k}
$$

If we utilize the state estimates of the observer to emulate full state feedback, and then combine the expressions for the system and the observer, we can write an expression for the error state. We define the error state $\tilde{x}$ as follows: $\tilde{x}=x-\hat{x}$. Using this definition, Equations (3.5) and (3.7) reduce to the following simple expression:

$$
\tilde{x}_{k+1}=[\Phi-K C] \tilde{x}_{k}
$$

The effective $\Phi$ matrix determining the observer error dynamics is the following: $\Phi_{o b s}=$ $[\Phi-K C]$. General guidance governing pole placement is to place observer poles (the eigenvalues of the $\Phi_{o b s}$ matrix) at least four or five times faster than the closed loop system poles (eigenvalues of the $[\Phi-K C]$ matrix) $[8]$ and make them critically damped. For a discretetime system this means that the poles will be on the real axis of the Z-plane and closer to the origin an appropriate distance. 
Using the actual states and the error states we can express the state equations in a form which utilizes a new, effective $\Phi$ matrix. The combined equations are presented below in condensed form:

$$
\left[\begin{array}{c}
x_{k+1} \\
\tilde{x}_{k+1}
\end{array}\right]=\left[\begin{array}{cc}
\Phi-\Gamma L & \Gamma L \\
0 & \Phi-K C
\end{array}\right]\left[\begin{array}{c}
x_{k} \\
\tilde{x}_{k}
\end{array}\right]
$$

The combined $\Phi$ matrix now contains the closed loop observer eigenvalues and the closed loop system eigenvalues. The ability to combine these systems and yet retain the original eigenvalues of the separate systems is referred to as the Eigenvalue Separation Theorem [12]. This very powerful idea is what allows us to solve independently for the system feedback gain and observer gain matrices.

\subsubsection{Stochastic Considerations in Observer Design}

Because one of our objectives in using an observer is to achieve a reduction in measurement noise, it is important to comment on some of the basic stochastic properties of measurement noise.

Gelb points out in [13] that if there are a number of sources contributing to an overall noise level, there is a very high likelihood that the resulting distribution will be gaussian. In the event that there is a bias in the distribution, a new mean can be defined and the bias can be added or subtracted after processing.

In the case of position measurements from the SHARPS acoustic position system or the DEL-NORTE RF system, the noise has been well characterized by field tests. Figure (3-5) illustrates the measurement noise distribution for SHARPS when receivers are mounted 70 meters from the transducer. In the case of the DEL-NORTE system, Figure (3-6) shows the distribution of errors in the measurement of a fixed range of 7 kilometers. Although one of the DEL-NORTE transponders illustrated a slightly biased error distribution function, both plot sets confirm the basic zero mean, gaussian distribution of the noise.

The importance of being able to characterize the measurement noise as having a gaussian distribution is that we have tools available to help build observers which minimize the noise in a least squares sense. Even though we don't know what the noise in each of the measurements will be, we do know that in the case of measuring a constant state, over time, the average 


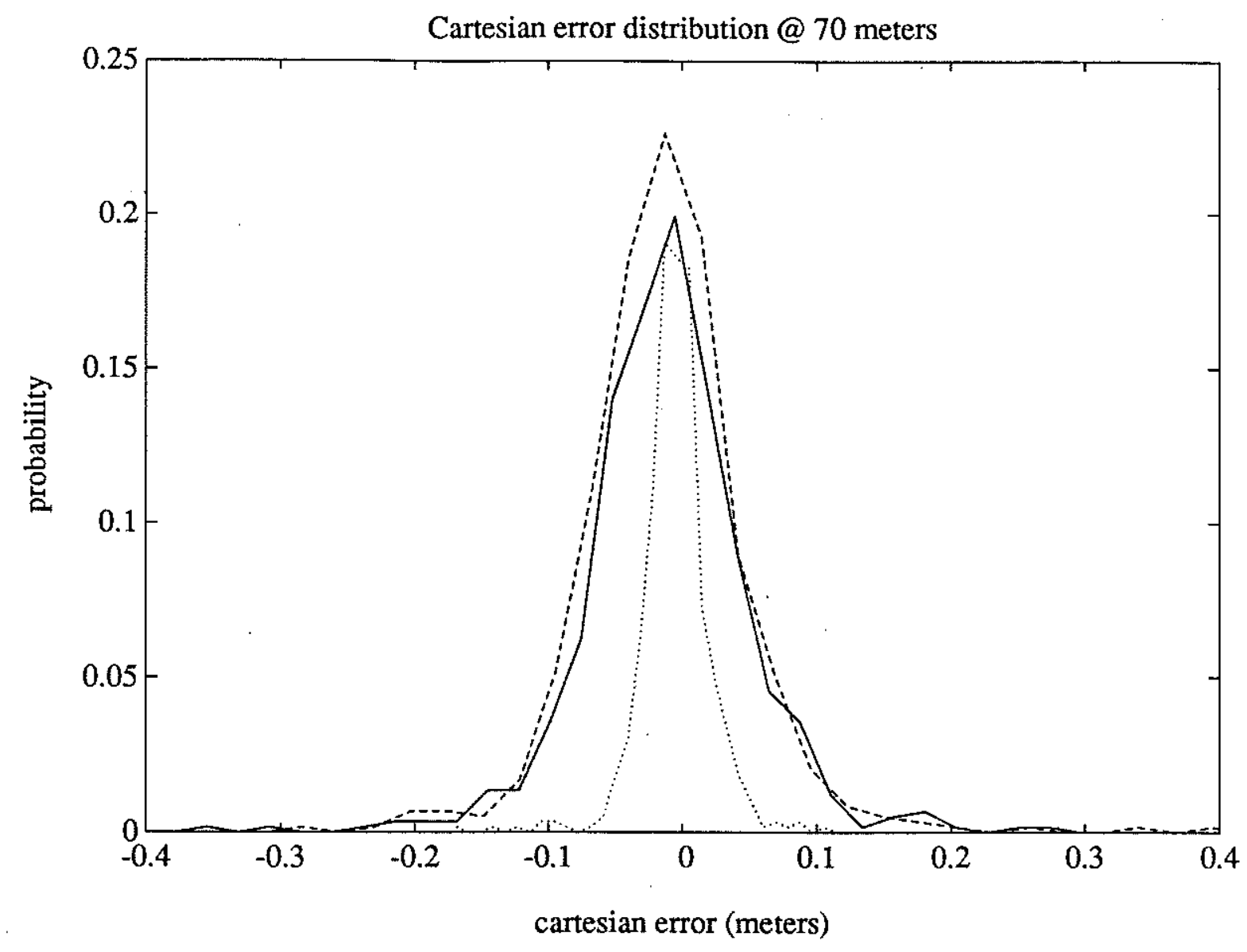

Figure 3-5: Actual sensor noise distribution for acoustic position transceivers.

of the measurements will approach the actual state. It also means that the probability distribution behaves as a well understood mathematical function with a known variance.

For reasons that will become clear in the next section we can also characterize system uncertainty as a random process noise with a gaussian distribution. This process noise, then, also has a characteristic variance which is important to the formulation of the observer.

There is an important caveat in the characterization of sensor noise. A discussion of sensor noise is not complete unless the random em bad fix is mentioned. A bad fix is one which is clearly out of the realm of possibility. Bad fixes may result from interruption of the acoustic path in the case of acoustic systems, for example, and are not accurately modeled as gaussian processes. In the 'hybrid' state estimators proposed, prefiltering is used to remove 


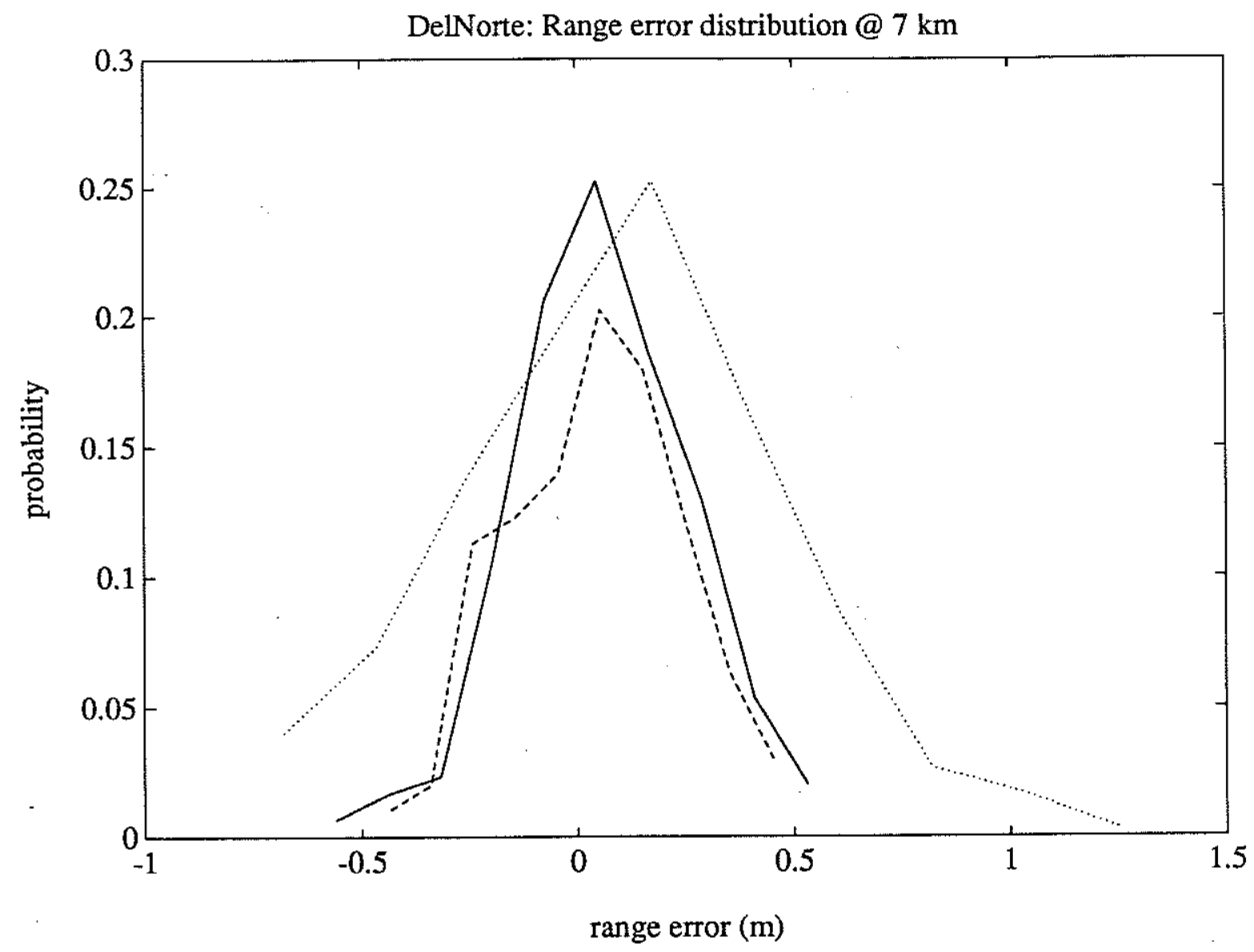

Figure 3-6: Actual sensor noise distribution for Del Norțe RF sensors.

them altogether. This topic is discussed in greater detail in later chapters.

\subsubsection{Optimal Linear Observers: Kalman Filters}

For a linear system with gaussian noise characteristics, the Kalman filter is the optimum observer. Assuming the characterizations of the measurement noise and the process noise are correct, the Kalman filter will provide the best estimate of the states in a least squares sense.

Although we have talked about noise reduction, it is not yet clear how we accomplish it. The first step is to determine a 'cost' function which we can minimize. We want to minimize the expectation of the error squared. Mathematically we can express this using the $\tilde{x}$ introduced in the previous paragraphs. 
Minimize the following:

$$
E\left(\tilde{x}^{2}\right)=E((\hat{x}-x)(\hat{x}-x))
$$

\subsubsection{Kalman Filter Dynamics}

The Kalman filter is a recursive filter and is perhaps best explained by the flow chart in Figure (3-7):

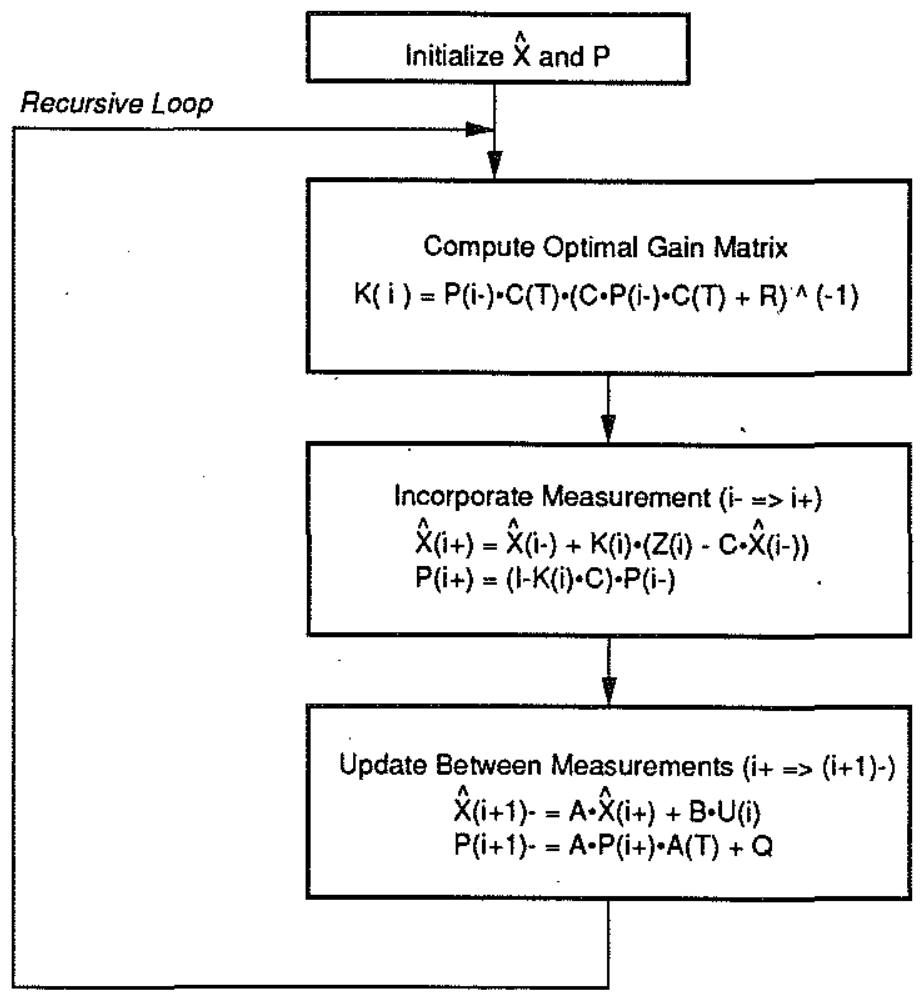

Figure 3-7: Recursive Loop for a Kalman Filter assuming a single measurement source.

The Kalman filter takes advantage of the measurements as soon as they are available. As illustrated in Figure (3-7), there are three distinct phases which distinguish the different sets of equations. These phases are determined by their timing relative to the actual measurements. The three phases are:

1. just prior to measurement $\left(i^{-}\right)$

2. just after measurement $\left(i^{+}\right)$ 
3. extrapolation to just before next measurement $\left((i+1)^{-}\right)$

The equations illustrated in Figure (3-7) describe the transition between these phases.

The process is started by picking initial values for the states and for the error covariance matrix. Using these initial values an initial Kalman gain matrix is calculated. Thereafter, each measurement starts a new transit of the loop.

The first step is the actual incorporation of the measurement $\left(i^{+} \leftarrow i^{-}\right)$. Here we use the precomputed value for the Kalman gain matrix $K$ and the actual measurement to compute the new state estimate. Here we also update the value of the error covariance matrix from $P_{i-}$ to $P_{i+}$.

In the next step we extrapolate the state estimates and the error covariance to the time of the next measurement $\left((i+1)^{-} \leftarrow i^{+}\right)$. To extrapolate the state estimate to the next measurement we use our model of the vehicle dynamics. For the update of our error covariance matrix, we use the knowledge of the "process noise" contained in $Q$ and the dynamics in the open loop $\Phi$ matrix. At the end of this stage we are ready to compute the new Kalman gain matrix to be used with the next measurement.

The final step is the computation of the gains. Once the Kalman gain matrix is updated, the system is ready for the next measurement. The gain matrix calculated now retains the history of all previous measurements.

\subsubsection{Steady State Kalman Filter}

As can be imagined, the first few measurements have a significant impact on the computation of the Kalman gain matrix. As time goes by and more measurements come in, the computation of the gain matrix becomes less and less sensitive to new measurements. If certain conditions are met, the gain matrix approaches a steady state value as the number of measurements made approaches infinity. Practically, this steady state may be reached after a relatively short period of time.

The steady state Kalman filter is often called a Wiener filter. The requirements to treat the Kalman Filter as a Wiener filter are listed in [13] and [14]. Further discussion of the steady state Kalman filter is left to the simulation discussion in Chapter 6. 


\section{Chapter 4}

\section{Utilization of Strapdown Inertial Measurements for JASON JUNIOR}

In an attempt to increase the control bandwidth of the ROV, JASON JUNIOR, accelerometers integrated in an attitude package were used to measure inertial accelerations of the vehicle. This package was used as the test platform for the design of the larger ROV, JASON. Figure (4-1) is an illustration of the actual attitude package removed from its aluminum pressure housing.

This chapter discusses the principles involved in inertial navigation, the peculiarities associated with strapdown inertial measurements and the implementation issues and errors which result from using SIMS on JASON JUNIOR.

A discussion of the control aspects of using the resulting inertial information is covered in the next chapter.

\subsection{Inertial Navigation System Basics}

The basic purpose of any inertial navigation system is to convert measured accelerations into a position time history in some acceptable frame of reference. A basic block diagram of a 'stable platform' system is presented in Figure (4-2). Stable platform refers to the fact that the table on which the accelerometers are mounted is maintained level with respect to the local vertical and oriented North (in lower latitudes). Under these conditions the accelerometers sense accelerations which are uncontaminated by the gravity vector and are 


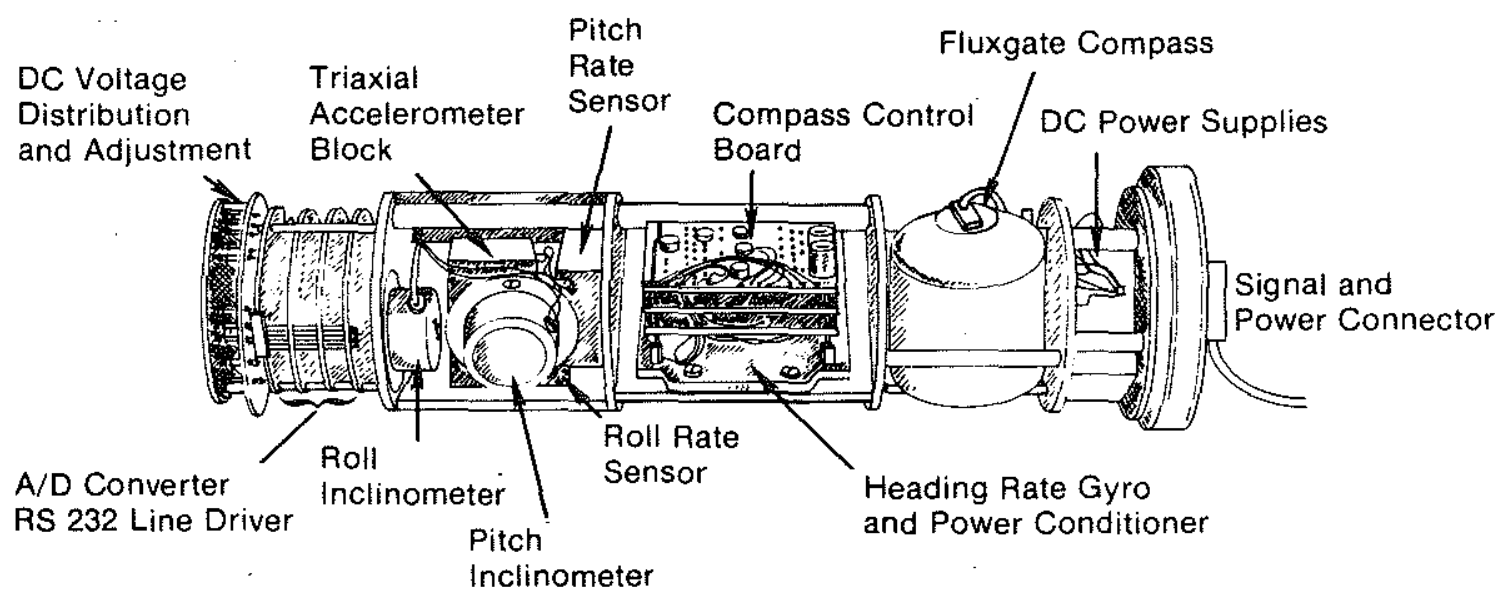

Figure 4-1: The accelerometer and attitude sensor package of JASON JUNIOR shown removed from its pressure housing. When installed in the pressure housing. the entire unit is approximately 23 inches in length.

directly related to the geographic coordinate system.

Typically, an inertial navigation system uses a stable reference platform on which to mount its accelerometers and gyroscopes. This platform is gimballed and driven to maintain the proper attitude by torque motors on each gimbal axis. The angular rate information from the gyros is used to drive the motors to null out the sensed angular motion [4]. This implementation inertially stabilizes the accelerometers and gyros on the platform from the motion of the vehicle. The resultant accelerometer outputs are then integrated twice, with an appropriate gravity correction, to transform the specific-force measurements into position information [4].

For the strapdown system, the accelerometer outputs are in body fixed coordinates and are not stabilized with respect to the motion of the vehicle. This means the measurements include specific-force and angular rate information with respect to the inertial frame, but in 
Initial

Conditions

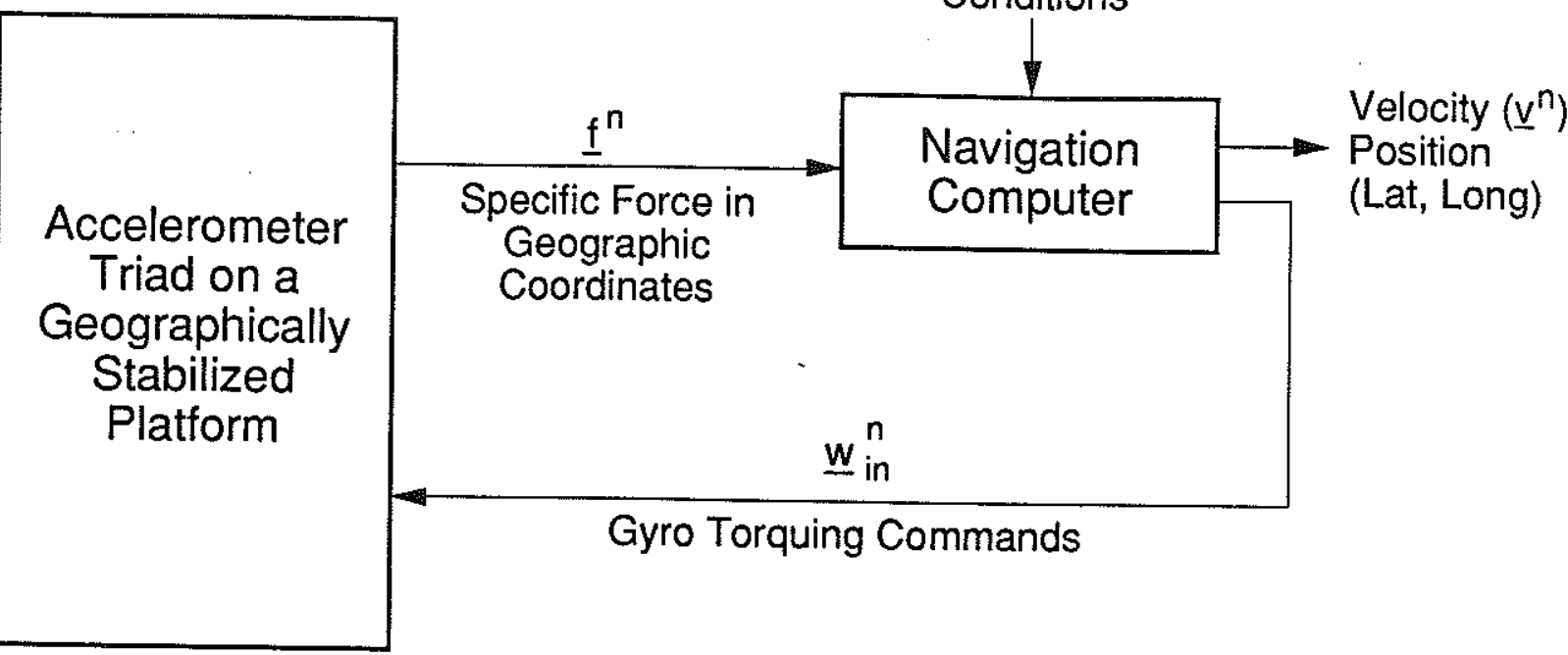

Figure 4-2: A conceptual block diagram which implements a stable platform inertial navigation system.

the coordinate frame of the vehicle body axes [4]. This requires that a recursive algorithm be employed to accurately measure the changing attitude of the vehicle. The attitude information is then used in a coordinate transformation matrix to convert from the body-fixed to the inertial coordinate frames.

An elementary block diagram of the strapdown inertial navigation system is included as figure (4-3). Note that the system shown is a system operating in local-vertical coordinates. Since geographic coordinates are the desired output, this is probably a typical implementation [4].

\subsubsection{Coordinate Frames}

The inertial navigation system may output information relative to one of many different coordinate systems. Visualizing the relationship between them is important, but may be difficult. Figure (4-4) illustrates the ordered rotations of the coordinate frame required to 


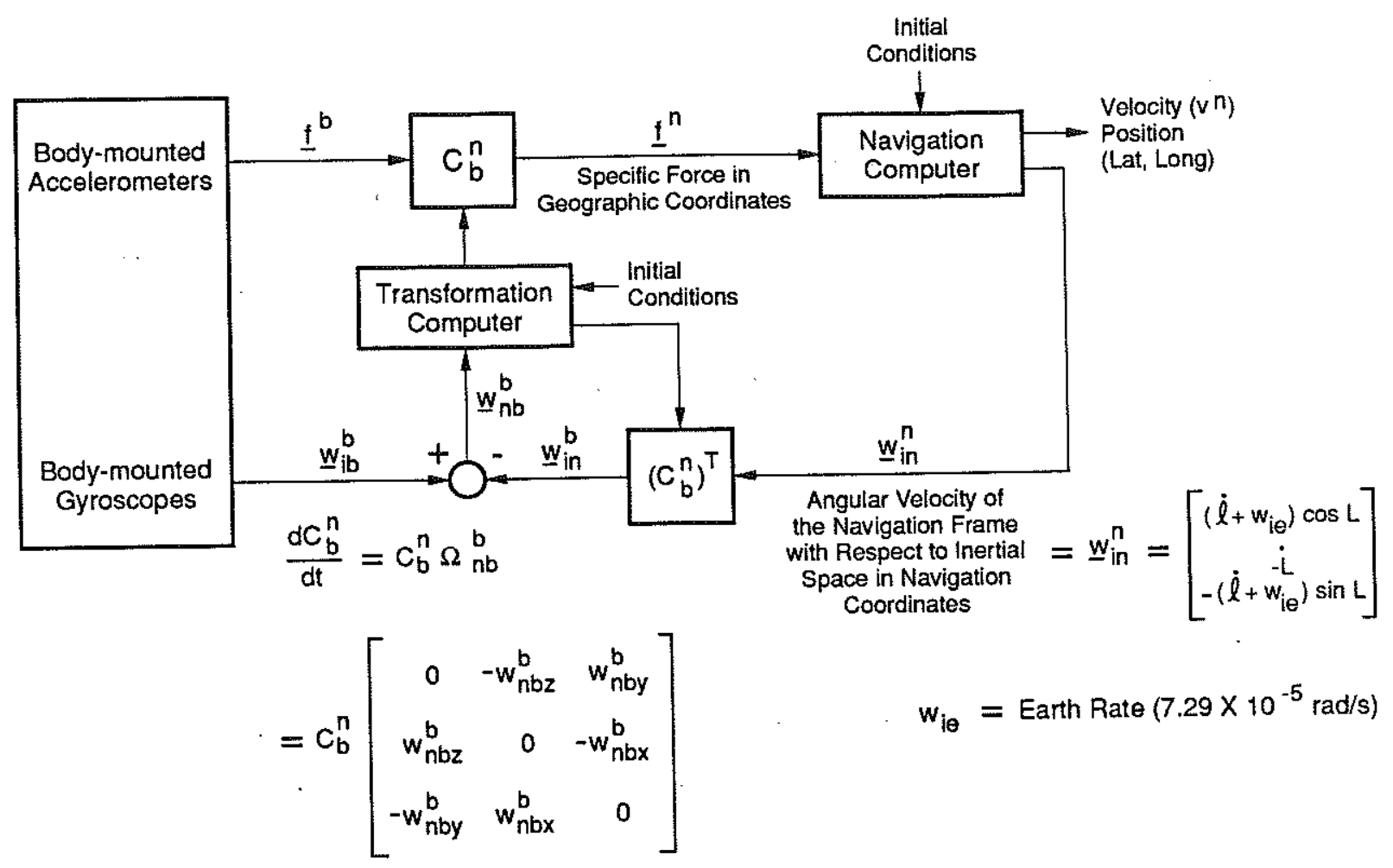

Figure 4-3: A mechanization of the strapdown inertial navigation system which operates in local-vertical geographic coordinates.

go from the local-vertical, geographic coordinate frame to the body fixed coordinate frame. The angles through which the coordinate frame must be rotated at each stage are called the Euler angles.

The importance of knowing the relationship between coordinate frames is that it allows us to perform a translation of acceleration measured in one frame to another. Euler angles formalize the process. Coordinate conversion for JASON JUNIOR is discussed in detail later in this chapter.

\subsubsection{Inertial Sensors}

In order to sense acceleration in whatever coordinate system we are using, high precision linear accelerometers are used for each axis of interest. If the accelerometers are mounted on a stable platform, fixed in some coordinate frame, then the accelerations measured can be used almost directly. This is not the case for the strapdown system. 

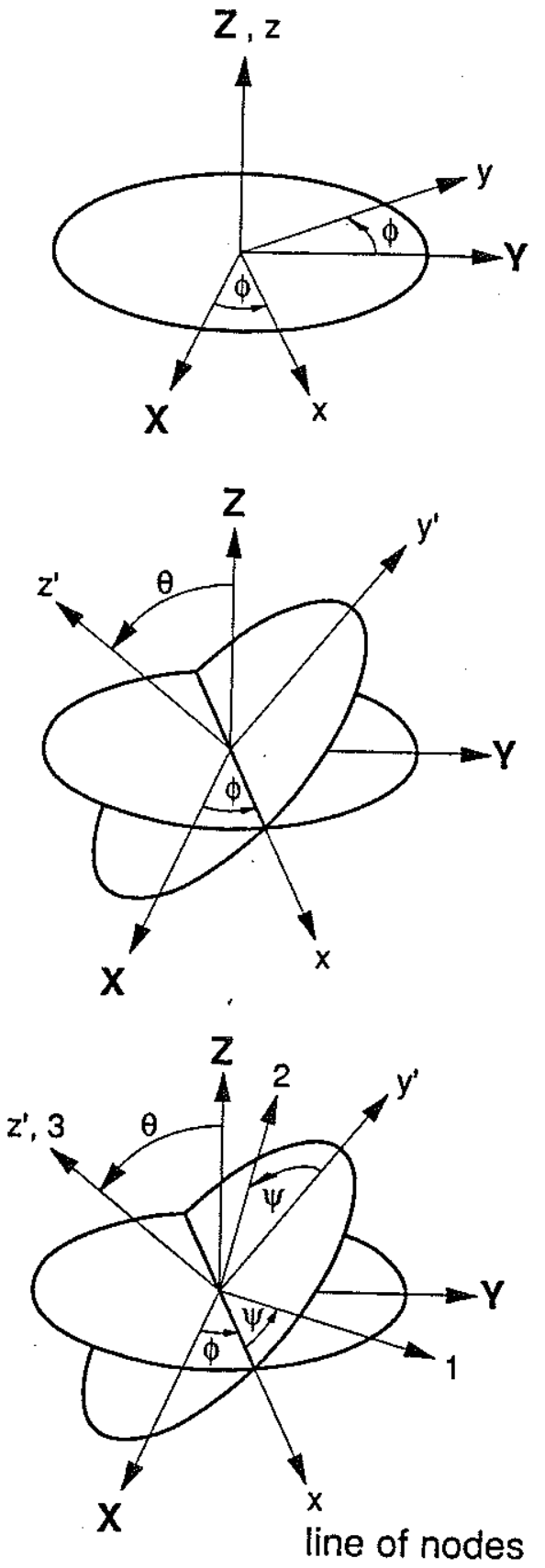

Figure 4-4: The three ordered rotations necessary to transform the geographic coordinate frame into the body referenced coordinate frame. (Neither the labeling of the angles nor the axes is standard and may vary from text to text.) 
For either system, the acceleration sensors used are most often pendulous torque-tobalance accelerometers [15]. Depending on their application they are either fluid filled or dry. The fluid filling gives the precision accelerometers a degree of shock protection and resonance damping. However damping can also be achieved electromagnetically for dry units.

If using a stable platform, the torquer motors for the gimbals are driven by angular motion measured by the gyros mounted on the table. In other words the table is moved to null the gyro outputs. (For a local-vertical stabilized system earth rate torquing must be performed to maintain the platform horizontal with respect to the local vertical.) Almost as an afterthought the gimbal angles can be read to measure the attitude of the vehicle. This luxury is not available to the strapdown system. The strapdown system must have the capability of measuring angle and angular rate in the three body fixed axes.

For the strapdown inertial navigator, attitude information is required to be able to transform the acceleration information into geographic position. For this system, then, the attitude sensors and algorithms become a critical ingredient in the success of the system.

To obtain attitude measurements, systems may use a variety of sensors, but typically single axis integrating rate gyros are employed for the axes of interest. For the stabilized system, as long as the angle between the platform and the local horizontal is very small, the angular displacements can be used directly to drive the torquing coils to align the platform. Solution of Euler's equations does not generally become an issue for the stabilized system of a ship or submarine.

\subsection{Strapdown Inertial Measurement Systems (SIMS)}

This section explains the configuration and operation of the Strapdown Inertial Measurement System (SIMS) as implemented on JASON JUNIOR. We start with a discussion of the basic difference between a stable platform system and a SIMS. Next, each of the system sensors utilized in JASON JUNIOR is covered. The section ends with a discussion of the algorithms used to implement the necessary operations and conversions to output geographic position and velocity information. 


\subsubsection{How SIMS is different}

As its name implies, a strapdown inertial measurement system is different from its stable platform relative. The position and orientation of the accelerometers in this system are fixed with respect to the body of the vehicle. As a result of this fixed condition, accelerometer measurements are contaminated by the gravity vector. If the system is not mounted at the center of gravity, the accelerometer outputs are also contaminated by radial velocities and accelerations. The equations presented below illustrate the factors which corrupt the measurement [16]:

$$
\begin{aligned}
& \alpha_{x}=u+q w-v r+a_{x}\left(-q^{2}-r^{2}\right)+a_{y}(-r+p q)+a_{z}(q+p r)+g \sin \theta \\
& \alpha_{y}=v-p w+u r+a_{x}(r+q p)+a_{y}\left(-p^{2}-r^{2}\right) a_{z}(-p+q r)-g \sin \phi \cos \theta \\
& \alpha_{z}=w+p v-q u+a_{x}(-q+r p)+a_{y}(p+q r)+a_{z}\left(-p^{2}-q^{2}\right)+g \cos \theta \sin \phi
\end{aligned}
$$

The left side of each equation is the output of the accelerometer. The right side is a combination of actual inertial accelerations $\left(a_{x}, a_{y}\right.$, and $\left.a_{z}\right)$, gravity vector contamination, and accelerations induced in the sensor by its displacement from the center of gravity. The $p, q$ and $r$ terms are the angular velocities in the body-fixed reference system and the angles $\theta$ and $\phi$ are two of the three Euler angles.

The obvious advantage of the strapdown system is that it eliminates the 'stable platform' and its associated servos, synchros and torquers. The advantage of this reduction in number of moving parts is offset, however, by the complex euler angle calculations required to track the attitude of the vehicle. Small angle approximations are not necessarily valid for the case of the strapdown sensors.

\subsubsection{Hardware and Sensors}

The equations of the previous section demonstrate the requirement for several measured quantities in addition to body fixed acceleration measurements. We must be able to convert the measured accelerations into inertial ones. Two events, implied in previous paragraphs, are required. The first event is removal of gravity vector contamination and the second is removal of effects caused by linear and angular velocities of the vehicle. Both events require 
an up to date determination of vehicle attitude.

The actual sensors employed on JASON JUNIOR are reviewed in the following paragraphs.

\section{Linear Accelerometers}

The linear accelerometers installed in the SIMS are the model QA- 1400, Sundstrand Data Control, 'Q-Flex' servo accelerometers. These are the pendulous torque-balance type with an integrated self monitoring loop. According to the contractor supplied literature, this internal servo system is used to monitor the output and correct for initial bias, scale factor and axis misalignment errors and their sensitivities to temperatures within a specified range. The servo system uses a fourth order dynamic model to achieve its performance.

The range of the accelerometer output is chosen by the user by virtue of his selection of load resistors. A photograph of the sensor and a simplified block diagram is included as Figure (4-5).

\section{Inclinometers}

The two inclinometers installed in the SIMS are Sperry pendulous inclinometers which have a $\pm 30^{\circ}$. range. Significantly, they have a maximum bandwidth of only $.5 \mathrm{HZ}$. (According to the current supplier of the inclinometer, the previously published bandwidth of $1 \mathrm{HZ}$ was too

optimistic.) Because of their narrow frequency response, and because of their sensitivity to translational acceleration, these angle sensors must be augmented by the angular rate sensors discussed in the next paragraph.

The inclinometers are orthogonally mounted about midway in the instrument bottle in the same section as the single axis gyros and the accelerometers. They are oriented such that one is sensitive to roll and the other is sensitive to pitch.

\section{Angular Rate Sensors}

To measure angular velocity and to provide an additional angular measurement, two WATSON, single axis, angular rate sensors are also installed in the SIMS. These sensors use two 
DIMENSIONS IN MILLIMETERS
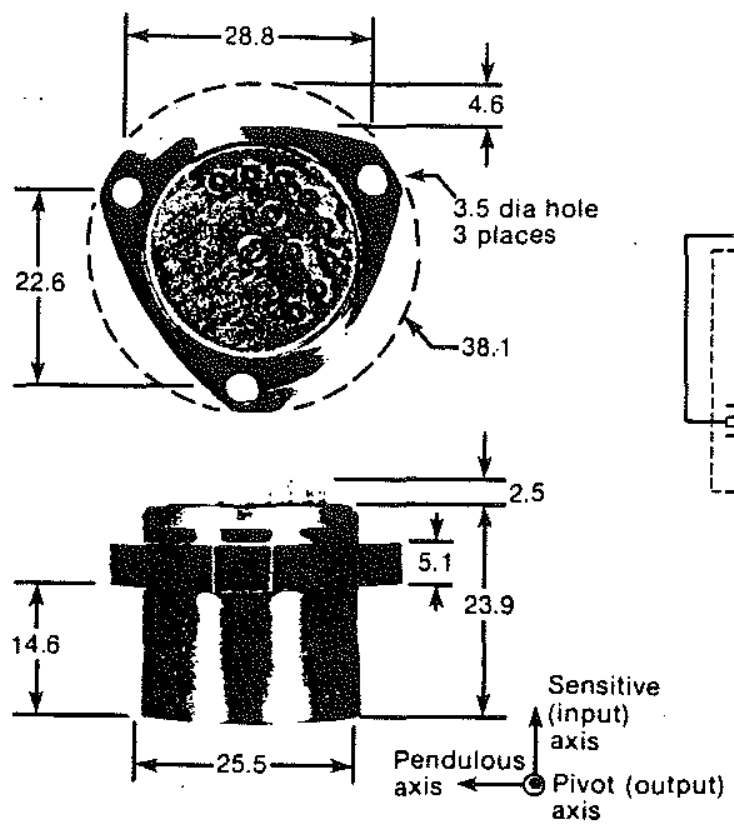

BLOCK DIAGRAM

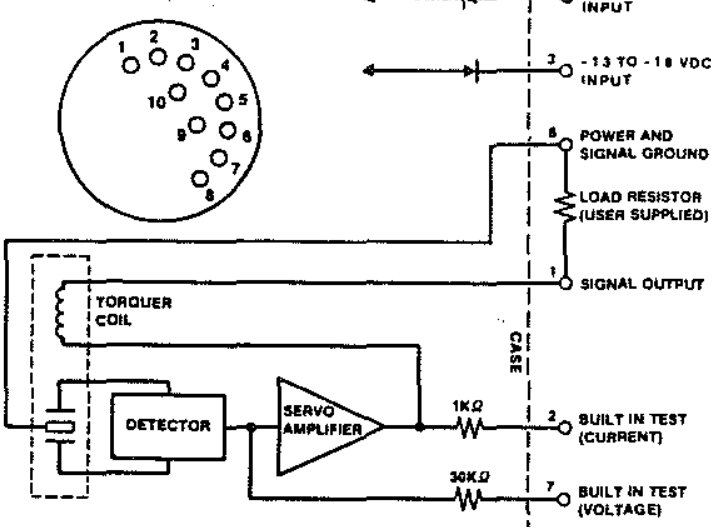

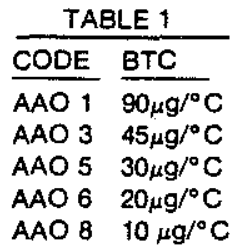

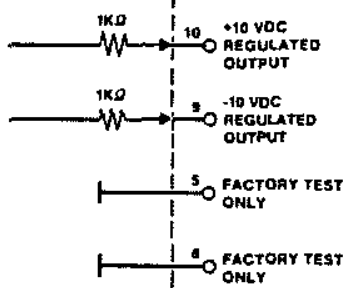

Figure 4-5: Two views and a simplified block diagram of the Sundstrand QA-1400 Q-flex Servo Accelerometer.

pairs of piezoelectric bender elements mounted in a small, lightweight enclosure and configured in a 'tuning fork' arrangement. A'pair' consists of two bender elements mounted end on end but one rotated 90 degrees from the other. The tuning fork arrangement is designed to cancel excitations caused by external vibrations or acoustic fields.

As referred to previously, these sensors are mounted approximately midway down the length of the bottle and in the same section as the majority of the other sensors. The output of the inclinometer and integral of the output of the rate sensor is combined to extend the overall frequency response of angular measurements. (The JASON ROV will take advantage of an instrument which combines both inclinometer and an integrated rate sensor in a single package.) 


\section{Heading Sensor}

The heading sensor is an ENDECO Type 869 Solid State flux gate magnetic compass. It uses a toroidal coil with dual pick off windings mounted on gimbals. The enclosure for the compass is fluid filled for damping purposes. This compass offers a choice of analog or 10 bit digital output. The corresponding resolutions are $\pm 0.5^{\circ}$ and $\pm 0.35^{\circ}$. The advertised accuracy of the compass is $\pm 1.0^{\circ}$. The gimbals provide stabilization for $\pm 30^{\circ}$ in pitch and roll.

Because the compass uses Magnetic North as its reference, we have been less than successful in using it with JASON JUNIOR in the steel test tank at the Coastal Research Laboratory at Woods Hole: What was used for heading reference in this application was a second transducer associated with the acoustic positioning system. This second transducer was mounted in line with the first on the top and centerline of the vehicle. Appropriate software changes permitted us to get a good heading reference as a real-time output of the positioning system.

\section{Heading Rate Sensor}

Unlike the Watson units, the heading rate sensor is simply a single axis gyro mounted such that its sensitive axis is the yaw axis. Its physical location in the SIMS is in the compartment to the left of the compass as shown in figure (4-1). The gyro, its power conditioner and the control board for the flux gate compass occupy this section.

The heading rate sensor is used to measure angular velocity and to extend the frequency response of heading measurements themselves.

\subsubsection{Attitude Determination}

Transforming the accelerometer output from the body fixed coordinate frame to a geographic coordinate frame requires that we have a relatively precise measure of the vehicle's attitude. The purpose of the additional sensors is to provide the measurements necessary to calculate the attitude in a recursive algorithm. This subsection discusses the algorithm and its implementation.

The necessity for the algorithm can be illustrated by the example of measuring pitch with various roll angles. If we were to measure the pitch of the vehicle with roll angle equal to zero, 
we would get an accurate measurement in the range of $\pm 30^{\circ}$. If, at the same time we wanted pitch, the vehicle was experiencing a $30^{\circ}$ roll, then the measured pitch would be less than actual because the gravity vector which actuates the pitch sensor is a much smaller component acting on the inclinometer's sensitive axis. In the extreme, the pitch sensor would have no sensitivity to pitch at all if the vehicle rolled to $\pm 90^{\circ}$. By the same token, accelerations in the heave (fore-aft) axis will contaminate the output of the inclinometer whose sensitive axis is pitch. This is true because the pendulous mass in the inclinometer will feel the acceleration in the heave axis.

Clearly then, the measurements made in one degree of freedom must be modified by the current measurements in other degrees of freedom. The relationship between angular velocities in inertial reference frame and body-fixed (Euler) reference frame is illustrated by the following equations:

$$
\begin{aligned}
\dot{\theta} & =p \cos \phi+q \sin \phi \\
\dot{\phi} & =-p \sin \phi \cot \theta+q \cos \phi \cot \theta+r \\
\dot{\psi} & =p \sin \phi \csc \theta-q \cos \phi \csc \theta
\end{aligned}
$$

These are coupled, nonlinear equations, where $p, q$, and $r$ represent the cartesian inertial reference frame angular velocities, and $\theta, \phi$, and $\psi$ are the Euler angles.

\subsubsection{Removing the Gravity Vector}

Once the actual attitude of the vehicle is determined, then we have sufficient information to separate the gravity vector from the acceleration measurement. Equations (4.1), (4.2) and (4.3), illustrated the relationship between gravity and the accelerometer outputs.

If the vehicle experiences a change in the gravity vector with time or position, then that variation would have to be included in an algorithm to determine the current gravitational force and direction. This additional consideration is most often associated with aircraft, missiles or spacecraft, which can experience large variations in the gravity vector due to changing altitude or changing orientation with respect to various heavenly bodies. 


\subsection{Implementation Issues}

There are many issues that are specific to the implementation of the SIMS on JASON JUNIOR. This section discusses the important ones.

\subsubsection{Physical Size}

Due to the size constraints of JASON JUNIOR, the original design for the attitude package was modified such that the SIMS would fit in a shorter pressure housing. This was accomplished by transferring a depth sensor (not shown in Figure (4-1)) to a separate and smaller pressure housing and connecting it via 'brantner' fittings to the SIMS. The smaller pressure housing was located on the opposite side of the vehicle. This not only shortened the SIMS, but also improved dynamic and static stability of the vehicle. The resulting SIMS pressure housing is approximately 22 inches long.

\subsubsection{Calculational Complexity}

Because of its location, the SIMS sensors are not collocated with the center of gravity. Because the SIMS sensors are spread along the bottle's length, each sensor is displaced differently from the center of gravity. The results are that each sensor is contaminated by a different moment arm which connects it to the center of gravity. Equations (4.1), (4.2) and (4.3) illustrate mathematically the affect of these contaminations.

\subsection{Sources of Error in SIMS Output}

A number of different factors contribute to the error in the output of the SIMS and its associated algorithms. The desired end product is a set of uncontaminated accelerations referenced to an inertial (or local-vertical) frame. Errors can result from imprecise measurements, noise, or approximations. We examine several sources in this section.

\subsubsection{Errors Due to Algorithm Selection}

In early work in digital inertial navigation systems and particularly in digital strapdown inertial systems, the primary concern was the error resulting from the numerical methods used in integrations and in the calculation of attitude [17]. The concern was to be able to 


\begin{tabular}{|l|r|r|}
\hline \hline \multicolumn{1}{|c|}{ Sensor } & \multicolumn{1}{c|}{ Resolution } & \multicolumn{1}{c|}{ Error } \\
\hline Accelerometer $(x$ and $y$ ) & .00012 g's & .000061 g's \\
Accelerometer $(z)$ & .00037 g's & .000183 g's \\
Inclinometer (pitch and roll) & $.02^{\circ}$ & $.01^{\circ}$ \\
Angular Rate (pitch and roll) & $.1^{\circ} / \mathrm{sec}$ & $.05^{\circ} / \mathrm{sec}$ \\
Heading & $.5^{\circ}$ & $.25^{\circ}$ \\
Heading Rate (gyro) & $.045^{\circ} / \mathrm{sec}$ & $.023^{\circ} / \mathrm{sec}$ \\
\hline \hline
\end{tabular}

Table 4.1: A list of the errors which result from just the resolution of the sensors. The least accurate of either a) the resolution of the sensor output or b) the resolution of the $A$ to $D$ conversion was used to fill the table.

make the expressions complex enough to limit the error and yet still be able to complete all calculations within the system's clock cycle. Such concerns are a much smaller problem today with faster computers and with optimized compiling of programming languages such as $C$. For the JASON JUNIOR implementation, the $10 \mathrm{HZ}$ update does not in general place a restriction on algorithm selection.

\subsubsection{Sensor Resolution}

Measurements of quantities of interest cannot be made any more accurately than that allowed by the resolution of the sensor. For example, if the output of the accelerometer is \pm 5 volts and it is processed by a 12 bit $A$ to $D$ converter, then the maximum resolution available is:

$$
\text { Resolution }_{a c c}=\frac{5 \text { volts }-(-) 5 \text { volts }}{2^{12}}=2.4414 \text { millivolts }
$$

If we multiply this resolution by the g's/volt conversion factor we get the maximum resolution of the accelerometer in units of specific force.

The resolution for the SIMS sensors have been calculated in this manner and are presented in Table (4.1).

Looking at the error associated with the resolution, we find that it is just one half the maximum resolution.

\subsubsection{Calibration and Alignment Errors}

Quite apart from sensor resolution, some errors are not associated with the capability of the sensor itself. A calibration procedure is required for the SIMS which can, in and of itself, 
introduce errors by not properly measuring the response of the sensors to excitation. This procedure can transmit any error in the equipment or procedure used for calibration to the output of the sensor. Any subsequent change in power supply voltage can also render a good calibration invalid.

Additional errors can result if, after calibration, the sensors and bottle are not properly aligned with respect to the vehicle. In theory, most of the alignment problems can be eliminated by calibrating the package once it has been placed on the vehicle. In practice, though, in situ calibration is very difficult to accomplish.

\subsubsection{Gyro Drift}

Some sensors, notably gyros, experience a drift in their output. This means that its accuracy varies with time and often in an unpredictable fashion. The heading rate sensor is vulnerable to this effect. Proper warm up of the instrument package is necessary to insure drift associated with temperature affects is not introduced.

\subsubsection{Gravity Vector Anomalies}

As mentioned previously, some circumstances require a separate calculation of the gravity vector. While altitude changes are a common cause in the change of the gravity vector in aircraft, a significant local phenomenon is required to affect the ROV.

\subsubsection{Approximations and Numerical Uncertainty}

Because many of the equations which describe the dynamics of the situation are nonlinear in the variable(s) of interest, a number of approximations are often made to facilitate the calculation of angles, angular velocities, attitude and ultimately inertial accelerations. As well, many simplifying assumptions are often made to allow reduction of the complexity and time required to make the calculations. In the context of available sensor resolution, this is not an issue. As higher resolution sensor outputs are avialable, and as update rates are increased above $10 \mathrm{HZ}$, however, this may become a problem.

The following chapter will examine the relative success of using the SIMS to augment position information. 


\section{Chapter 5}

\section{Hybrid State Estimation for JASON JUNIOR}

This chapter combines information from chapters two through four and describes the development of a "hybrid" state estimator for JASON JUNIOR. In our state estimator we are combining position and acceleration information. The combination of acceleration information and acoustic position information need not be restricted to JASON JUNIOR, however, and holds promise of significant benefits for other ROV's which utilize an acoustic net for control and navigation.

To be clear, there have been several successful demonstrations of ROV closed loop control with only position measurement provided by acoustic positioning systems $([3],[1])$. There have also been extremely accurate strapdown inertial navigation systems built which can provide high accuracy velocity and position measurements for long periods of time without update. The purpose of this approach is ultimately to make existing long baseline acoustic positioning systems viable for precise closed loop control of underwater vehicles with a minimal investment. The equipment required to facilitate this enhancement is remarkably compact and has relatively low power consumption.

The combination of sensors discussed in the paper presented by Paul Rushfeld at the 1987 Offshore Technology Conference [1] is a close example of what we wish to accomplish for JASON JUNIOR. What Rushfeld implied was that a highpass digital filter employed on the output of the accelerometers can make an inexpensive, unsophisticated system, perform well.

The underlying assumption stated by Rushfeld was that ROV's are not subject to constant 
accelerations for long durations. This means that constant outputs are as a result of biases or contamination. He also suggests that the integration constants be established by the position fixes. We will explore these ideas in more detail in this section. First, however, it should be pointed out that for the ROV's in our discussion, the contamination due to gravity is at the same frequency as vehicle roll and pitch and cannot be filtered out in the manner that Rushfeld suggests. Our system must explicitly account for gravity effects and remove them prior to utilization of the acceleration measurements.

\subsection{Objectives for the State Estimator on JASON JUNIOR}

The state estimator algorithm that we develop should use acoustic position and the inertial acceleration information in such a way as to provide for three basic levels of enhancement:

1. redundancy of state information,

2. improvement over current estimation dynamics, and

3. state estimation between acoustic fixes.

These three levels of enhancement are arranged in hierarchical order and translate into desired control improvements as explained in the following paragraphs.

\subsubsection{Redundancy in Measurements}

Redundancy in measurement has several advantages in and of itself. Initially, redundant information permits you to recover from missing measurements from one sensor or the other. A missing measurement might occur as a result of a mechanical, electrical or electronic failure. The most likely cause for a missing acoustic position measurement, however, would be rejection of the fix due to noise or interruption of the acoustic path. Prefiltering of the fix is necessary when a fix is returned which is not in the realm of physical possibility as determined by an appropriate state-varying metric. We call these bad fixes flyers. Although the number of these fixes is relatively small with a field proven acoustic positioning system, their presence can significantly effect control efforts. 'Flyers' do not fit the typically gaussian noise probability density distribution and can be more correctly thought of as having a uniform distribution. 
A result of redundant measurements is the reduction of noise in the state estimate. The Kalman filter, for example, utilizes the measured stochastic properties of the noise associated with each measurement and produces an estimate whose noise level is lower than either of the two measurements. The total equivalent noise level in the estimate is calculated in a manner similar to the calculation of equivalent resistance for resistors in parallel.

$$
\frac{1}{\sigma_{\text {total }}}=\frac{1}{\sigma_{1}}+\frac{1}{\sigma_{2}}+\cdots
$$

Analysis and treatment of 'flyers' requires different handling. Because of a flyer's uniform probability density function its probability distribution is not characterized by standard deviation measurements. Since the presence of a gaussian distribution is the basic assumption about the probability of measurement noise used in the development of the Kalman filter, 'flyers' must be rejected in a prefiltering step rather than being incorporated into the state estimate by the filter.

In general, the addition of new sensors should lower the noise in the estimate regardless of the noise level of the sensor (accelerometers) being added.

\subsubsection{Improved Estimator Dynamics}

The second level of improvement involves the dynamics of the estimator. As we discussed in Chapter 3, at longer ranges the position update rate available from an acoustic tracking system is limited by the acoustic travel time from pinger to receiver. Although we can increase the frequency of measurements by techniques such as range-gating or frequency modulation, the state measurement is delayed reaching the control system by at least the amount of time that it takes sound to travel from pinger to receiver. So, although measurement frequency is not mandated by acoustic travel time, the delay is. This delay manifests itself by adversely affecting the control bandwidth.

Control bandwidth is also affected by the noise level. Reductions in noise level will also improve the effective control bandwidth of the vehicle. 


\subsubsection{Long Term Estimation}

The next level of improvement to be achieved is the establishment of relatively long term state estimation through inertial measurements. Long term in this context is on the order of 5 seconds. Using a sound velocity of 1500 meters/second this would correspond to a distance of 7500 yards. A combination of state history and current acceleration measurements would be used to provide the best estimate of actual vehicle states.

We could also have considered this level to be an extreme case of the second level of improvement, where acoustic information is received at a much slower rate than the acceleration measurements. With the longer time between acoustic fixes, there is a greater likelihood of significant error developing in the position and velocity derived from the acceleration measurements.

\subsection{Available State Information for JASON JUNIOR}

\subsubsection{Acoustic Position Information}

The acoustic positioning system, SHARPS, provides high accuracy position information at a maximum rate of about 5 fixes per second in the test pool. We recall from Chapter 3 that update rate is a variable which depends on the environment and on the distance from the ROV to the transponders in the net. The maximum distance from the vehicle to the transponders being utilized is currently 100 meters. Figure (5-1) is an illustration of the components which make up this acoustic position tracking system.

\subsubsection{Strapdown Acceleration Measurements}

Decontaminated acceleration measurements that come from the attitude package go through coordinate transformations before being used in the state estimation process. The raw acceleration measurements are sampled at $10 \mathrm{HZ}$ and are accompanied by the outputs of two inclinometers, three angular rate sensors, and a flux-gate compass. True vertical is resolved (closely approximated) and the inertial accelerations are calculated for each instant. Computation of angular velocities, pitch, roll, yaw, and inertial accelerations is accomplished within one clock cycle. All raw sensor outputs are available for display or further computations. 


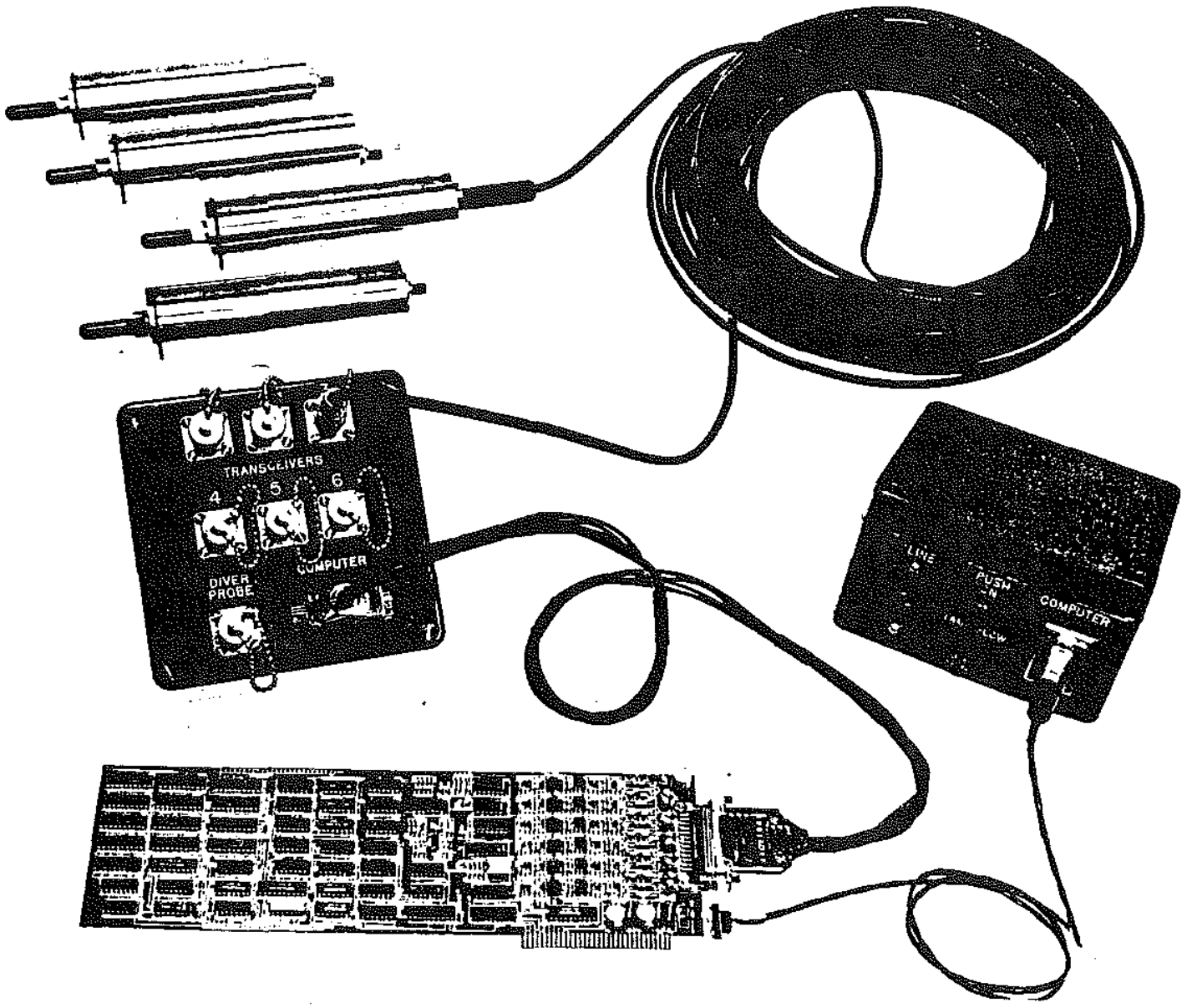

Figure 5-1: SHARPS components including transducers, cabling, junction box, power supply, and controller and data acquisition board.

\subsection{Use of the 'Hybrid' JASON JUNIOR Observer}

To accomplish the first objective, redundant state information, we have to convert the inertial acceleration measurements into position (and velocity) information. Once the information is transformed into the reference frame of interest, a straightforward integration algorithm is used to convert acceleration into postion and velocity. Although earlier works such as Broxmyer [18] and Pitman [19] both discuss the basic algorithms for accomplishing these integrations, strapdown inertial system references, such as Catford [16], and VanBronkhorst [20] are more germain.. 


\subsubsection{Preliminary Considerations}

A number of hurdles exist which must be overcome. The first is choosing the correct integration constants for the process. What is most often done is an initialization with JASON JUNIOR immobilized, so that neither velocity nor position are changing. Velocity is identically zero and position is chosen as the peak of the position probability curve developed with SHARPS measurements over a designated time interval.

Once the acoustic and inertial measurements are in equivalent forms, we must choose a method to combine them. The Kalman filter uses a weighting factor approach which is well suited to this application.

Measurement weighting factors in the Kalman filter are inversely related to the sensor noise levels. As a simple example, if acoustic and inertial measurement noise levels are equal, each weighting factor is 0.5 . However, if the acoustic position information is perfect (except for 'flyers' as discussed earlier), then acoustic fixes are weighted with a factor of 1.0 and inertial measurements are weighted with a factor of 0.0 , regardless of their own noise level.

This approach utilizes both acoustic and inertial position measurements, but has serious shortcomings. A single set of weighting coefficients can not account for all possible combinations of measurements:

- simultaneous acoustic and inertial measurements,

- inertial measurements during periods between valid acoustic fixes, and

- no measurements, whatever the reason.

Using a single set of coefficients, no information or wrong information will be used for the update in the event that a position fix drops out. Using the example of perfect acoustic measurements (again, except for 'flyers'), the presence of the acceleration-derived position would be ignored even in the absence of an acoustic position fix.

Clearly, something other than a single set of coefficients is required to fully utilize all of the available measurements. 


\subsubsection{Development of the 'Hybrid' Observer}

\section{A First Look}

One approach to combining available measurements is the use of two distinct update laws. One law is used when position fix information alone is available, and a second law is used when only acceleration-derived measurements are available. The second law utilizes model propogated estimates and acceleration-derived position. For this arrangement, the position estimate and the acceleration-derived position is 'reset' when a valid position fix is received.

The disadvantage of this approach is that the noise in the acoustic position measurement is passed to the estimate and the acceleration-derived positions in an unfiltered way. It could be argued that given the low noise levels experienced in our acoustic position measurement experience with SHARPS, this error propogation is not a significant problem. For longer range systems, however, the presumption of low noise levels is unwarranted. In fact, even for short range systems error propagation is a very significant problem in the event of a 'flyer'. At the least, such a system requires that the acoustic position fix be prefiltered with a moving velocity window or other construct to insure that the 'flyers,' are discarded.

If a Kalman filter is used for updating the state estimates with inertial and acoustic information, a reduction in the estimate's equivalent noise level is realized and is calculated using Equation (5.1). If the position estimate and the inertially- derived position measurement are arbitrarily reset by each valid acoustic fix, however, the reduction in equivalent noise level predicted by Equation (5.1) will not be achieved.

\section{A Better Observer}

A better observer retains the history of previous measurements and weights incoming measurements appropriately based on their validity. A measurement rejected by a prefilter, for example, has no validity. Then for each time steop there is the possibility of having two, one, or no measurements with which to update the estimate.

When both measurements are available (at the post prefilter stage) they are weighted based on their noise statistics and both used in the update of the state estimate propagated from the previous time step. Until or unless the filter reaches steady state, each measurement 
update also modifies the error covariance matrix and the Kalman gains in advance of the next time step. Because of anticipated growth in the error between actual and inertial position, some method must also be devised to reset the integration constants. A growing divergence will eventually bias the estimate and produce an error which continues to propagate. Remember that a basic assumption of our Kalman filter is that the noise has a zero mean. A quantitative and time varying metric is required to determine the appropriate time to call for a reset.

When only one measurement is available it is again combined with the state estimate propagated from the previous time step. Because the Kalman gain matrix calculations are based on the characteristics of the sensors, the gain matrices are different for each of the single measurements and different also from the two measurement case.

When there are no measurements available at the output of the prefilter, the estimate propagated from the previous time step receives no modification.

There are two primary issues. The first issue is the timing question. Figure (5-2) illustrates a flow path which allows the observer to utilize the measurements as they are available. The second issue is the mechanism or metric used to determine the appropriate time to reset the inertial information. Although our inertial acceleration measurements integrated for as long as 70 seconds showed no steep ramps in position error, resets must occur at some point in time. A logic tree for the inertial reset determination is presented in Figure (5-3).

The measure of success is how closely the estimated position tracks the actual position, however.

\subsubsection{Simulation Results}

To test the efficacy of the proposed observer structure, a single degree of freedom simulation was performed to see the affect of varying acoustic position measurement noise levels and lowering acoustic position update rates when inertial measurements were available. Performance comparisons between systems with and without inertial augmentation are available in the remaining figures of this chapter.

For the simulation, the observer was constructed using a linear description of the dynamics, while the actual state values were calculated using the nonlinear description from 


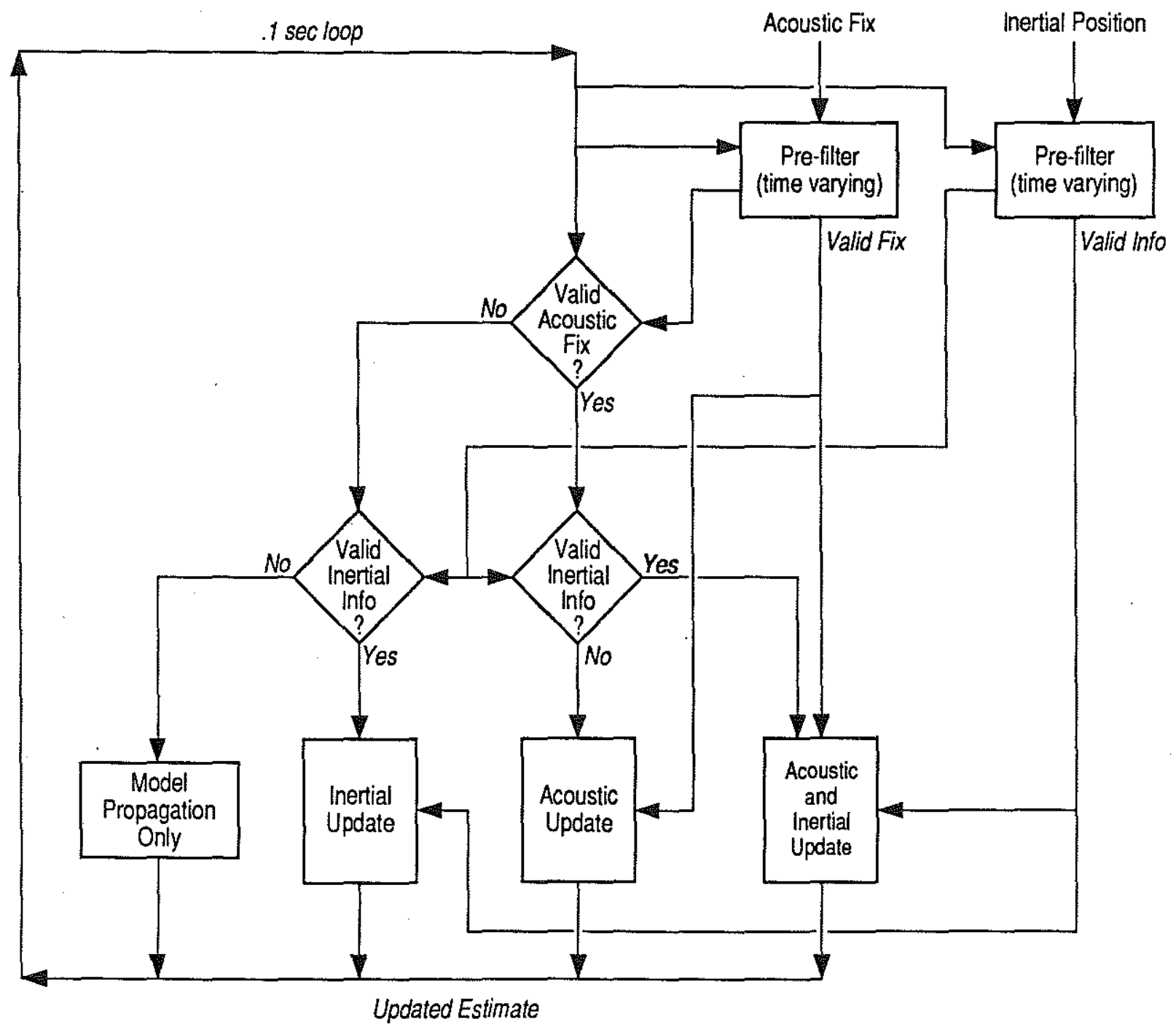

Figure 5-2: This diagram illustrates the timing sequence which enables the observer to take advantage of the measurements as they are available. 
Chapter 2. Again a fourth order Runge-Kutta approximation was used for the integration.

The target dynamics for the unaugmented systems were:

- Natural frequency of 1 radian/second,

- Damping Ratio of .7, and

- Estimator error dynamics critically damped with a natural frequency of 4 radians/second.

Kalman gain matrices for the 'hybrid estimators' were calculated assuming the filter was steady state. In all cases for these estimators, the inertial measurements were available every .1 seconds and had a noise level characterized by a standard deviation of .04 meters.

\section{Noise Rejection}

Inputting a desired sinusoidal trajectory with reasonable dynamics, system performance was measured as the acoustic noise level was varied. Simulation runs were made with and without inertial information in the state estimator. The results of these comparisons are displayed graphically in the remaining figures.

The first two graphs in figure (5-4) illustrate the differences between augmented and unaugmented system performance when acoustic measurements have very low noise (standard deviation of $2 \mathrm{~cm}$ ) and an update rate of $5 \mathrm{HZ}$. The upper graph compares desired and actual position while the bottom graph compares control action demanded by the two systems. This represents nearly a best case performance for an acoustic positioning system and is modeled after the SHARPS performance specifications.

Although there is no dramatic difference in the two systems' performance shown in figure (5-4), it is significant to note that there is a noticeable, if incremental, improvement when the inertial information is avialable.

Figure (5-5) does show a dramatic difference, especially in control action when inertial information augments the system. This figure illustrates acoustic position system operation at the same update rate, but at a noise level (standard deviation) of .1 meters. Other plots, such as those of desired versus actual velocity, are equally impressive.

The remaining two figures are a repeat of the previous two except that the acoustic update rate is $1 \mathrm{HZ}$. Here again, the dramatic improvements show up when the acoustic noise level 
is relativel high. Figure (5-7) might correspond to the characteristics of a $30 \mathrm{KHZ}$ acoustic position system operating at a range of just under 1500 meters.

The general conclusion to be drawn is that larger noise levels in the acoustic positioning system result in larger control actions, larger tracking errors, and a lower control bandwidth. For our purposes, the more important conclusion is that the inertial information is always helpful in improving system response regardless of the acoustic noise level. The improvement is particularly dramatic as acoustic noise level increases to a level at or above the noise level of inertial measurements.

\section{Countering the Effects of Lowering Update Rate}

The other comparison which is important here is system performance versus sampling rate, with and without acceleration measurements. The simulation comparisons allow us to compare performance and draw some conclusions about the influence of the inertial information on countering low update rate.

The two update rates chosen for these simulations were both slower than the inertial update rate, but still well above the bandwidth of the desired trajectory. For the cases of low acoustic noise levels, the additional information provided minor improvements in performance. For the case of low update rate and high noise level, the results are dramatic. (Though dramatic improvements were also seen when acoustic noise level was high with a 5 HZ update rate.)

Some additional simulations were performed which suggest that improvements in performance may be more noticeable with higher system bandwidths. At higher system bandwidths, however, the control action was excessively large and spiked, with and without inertial augmentation. The simulations illustrated represent more reasonable and realistic demands on the actuators.

\section{Summary of Results}

The performance of the augmented system appeared to be better in all practical applications. Minor improvement is seen at low acoustic noise levels, when the system is operating in best case, but large dividends are received when the acoustic noise level deteriorates and the 
acoustic update rate is lowered. More dramatic effects can be seen when changing noise levels. Both lower update rates and higher noise levels are encountered in longer range systems, though, and make the augmented system worthwhile. 


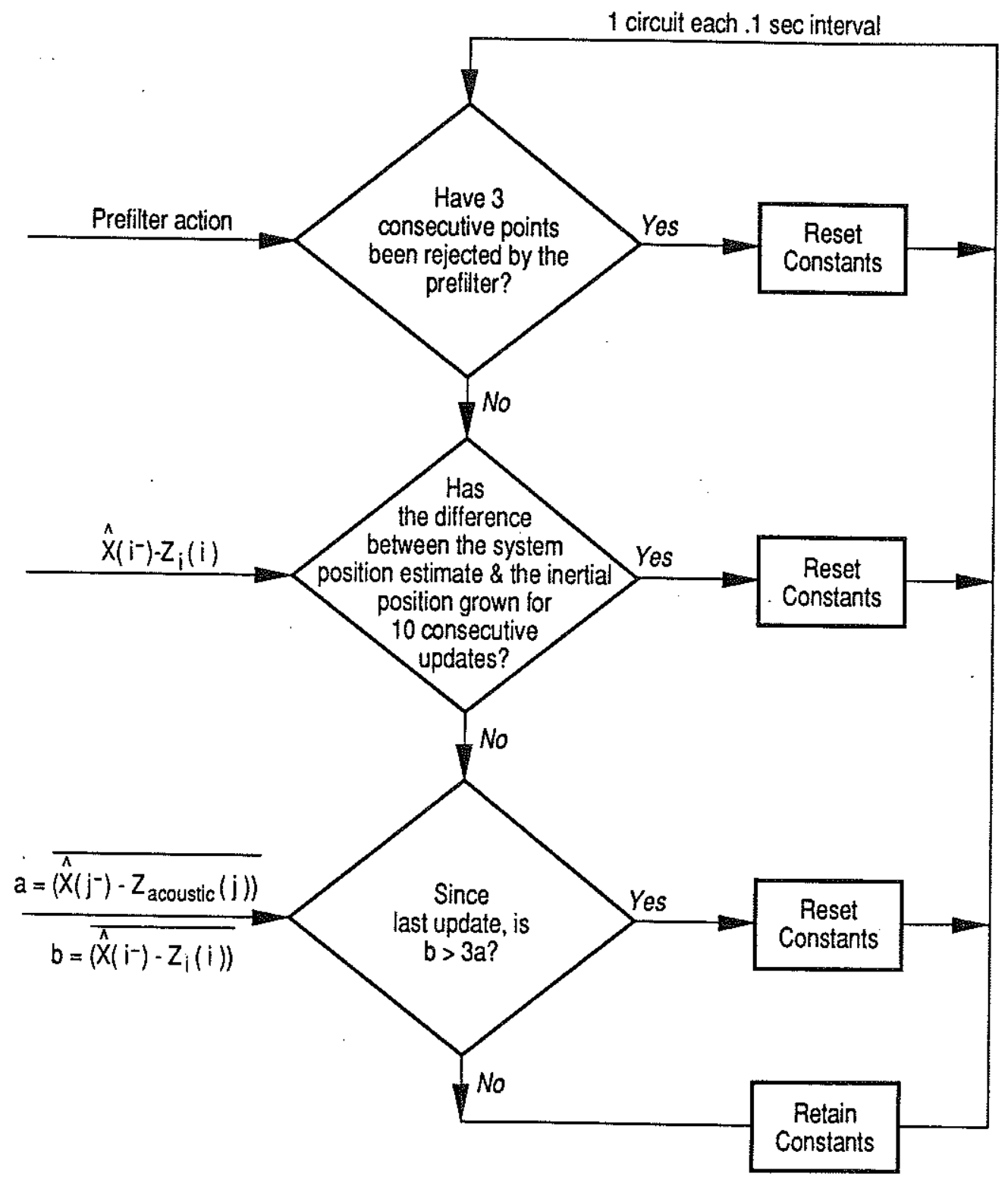

Figure 5-3: A logic is required to insure that the inertial information is utilized to its fullest extent, but does not corrupt the estimate if it drifts. 

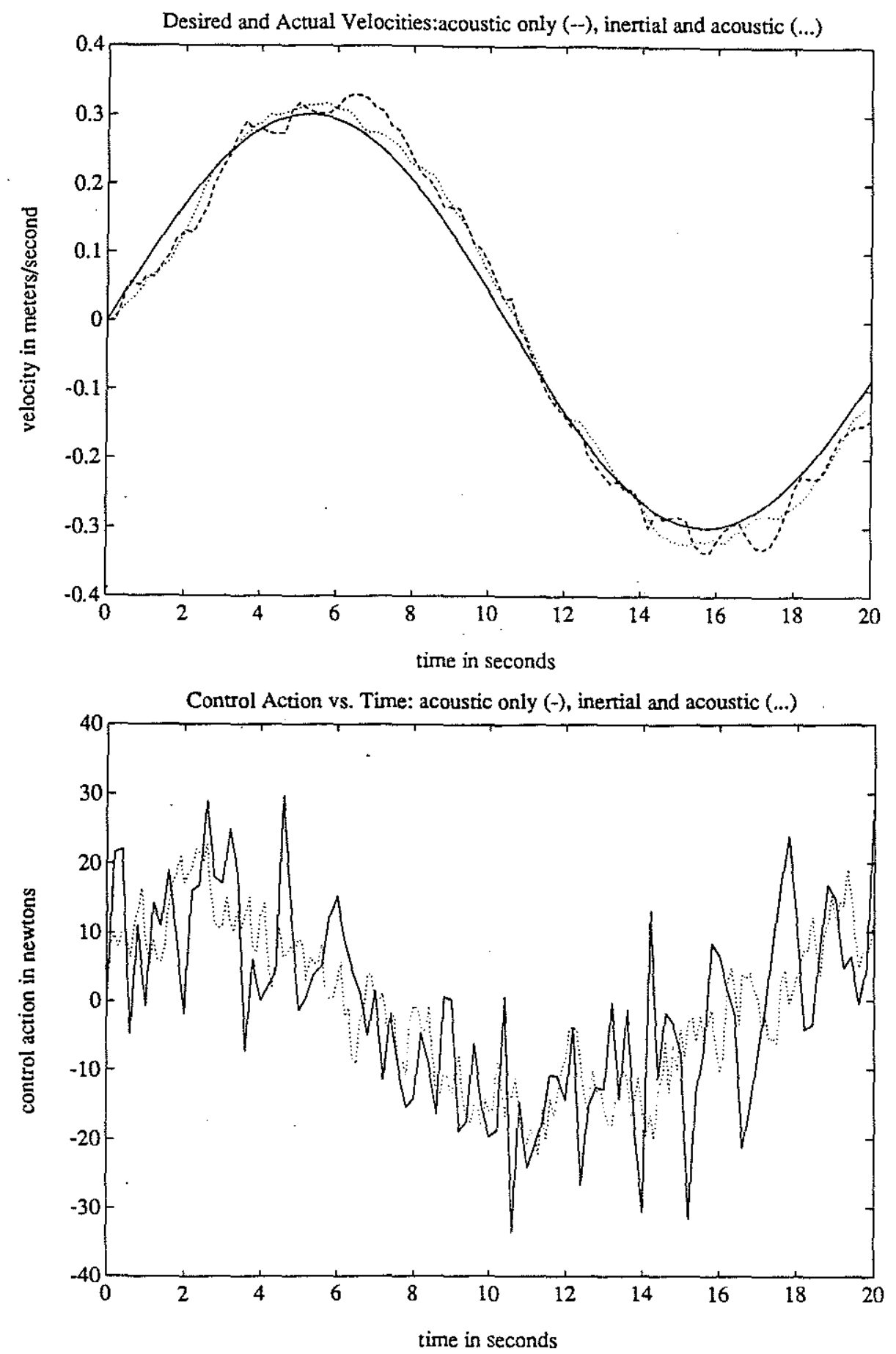

Figure 5-4: These graphs represents best case acoustic position system performance. Acoustic measurement noise level is .02 meters and measurement update rate is $5 \mathrm{HZ}$. A small improvement in trajectory following and control action reduction can be seen from these two curves. 

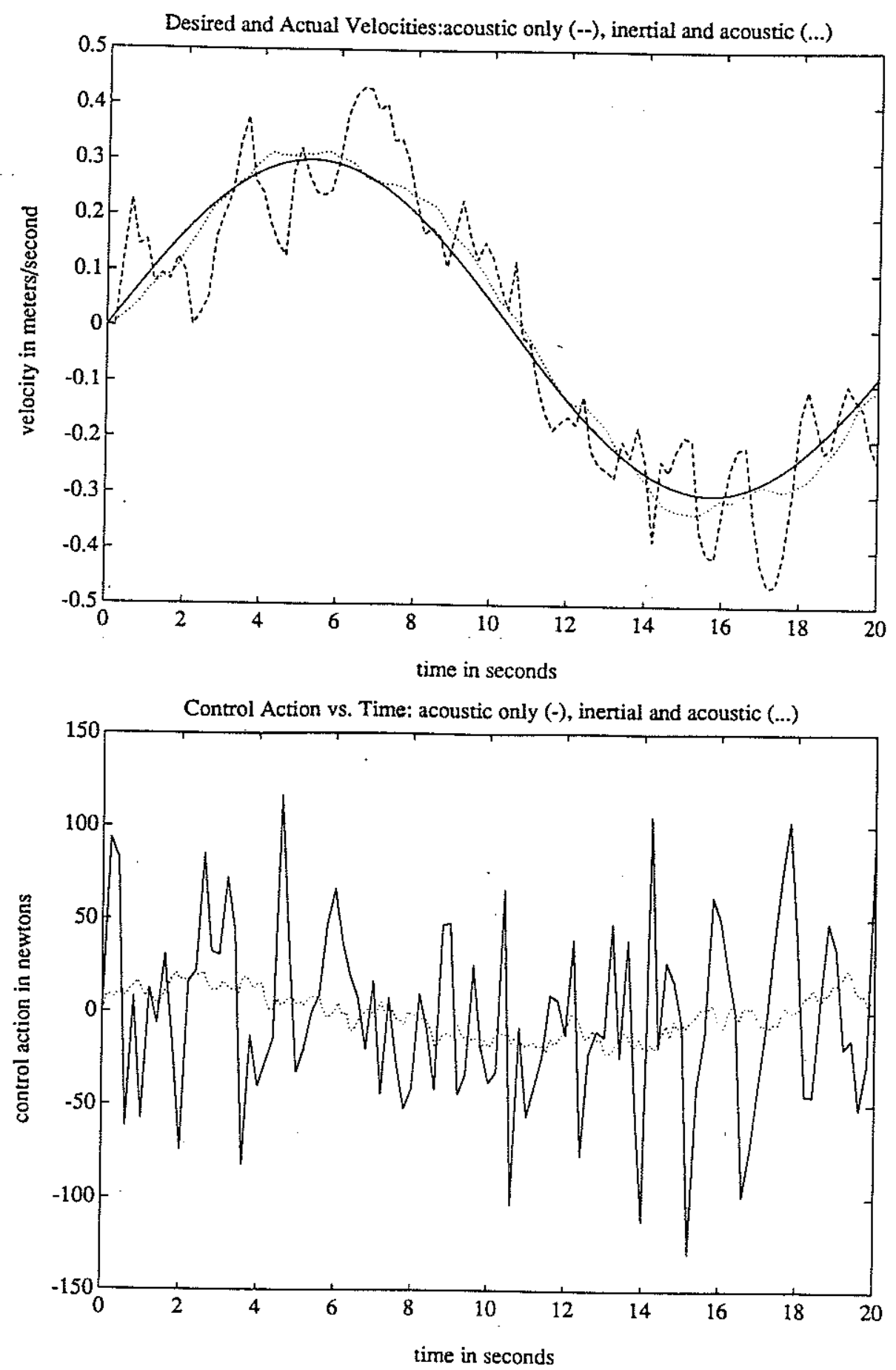

Figure 5-5: In case 2, the noise level in the acoustic measurements is increase to .1 meter. Dramatic differences are seen in all performance comparisons, but particularly in the velocity error and in the generated control actions. 

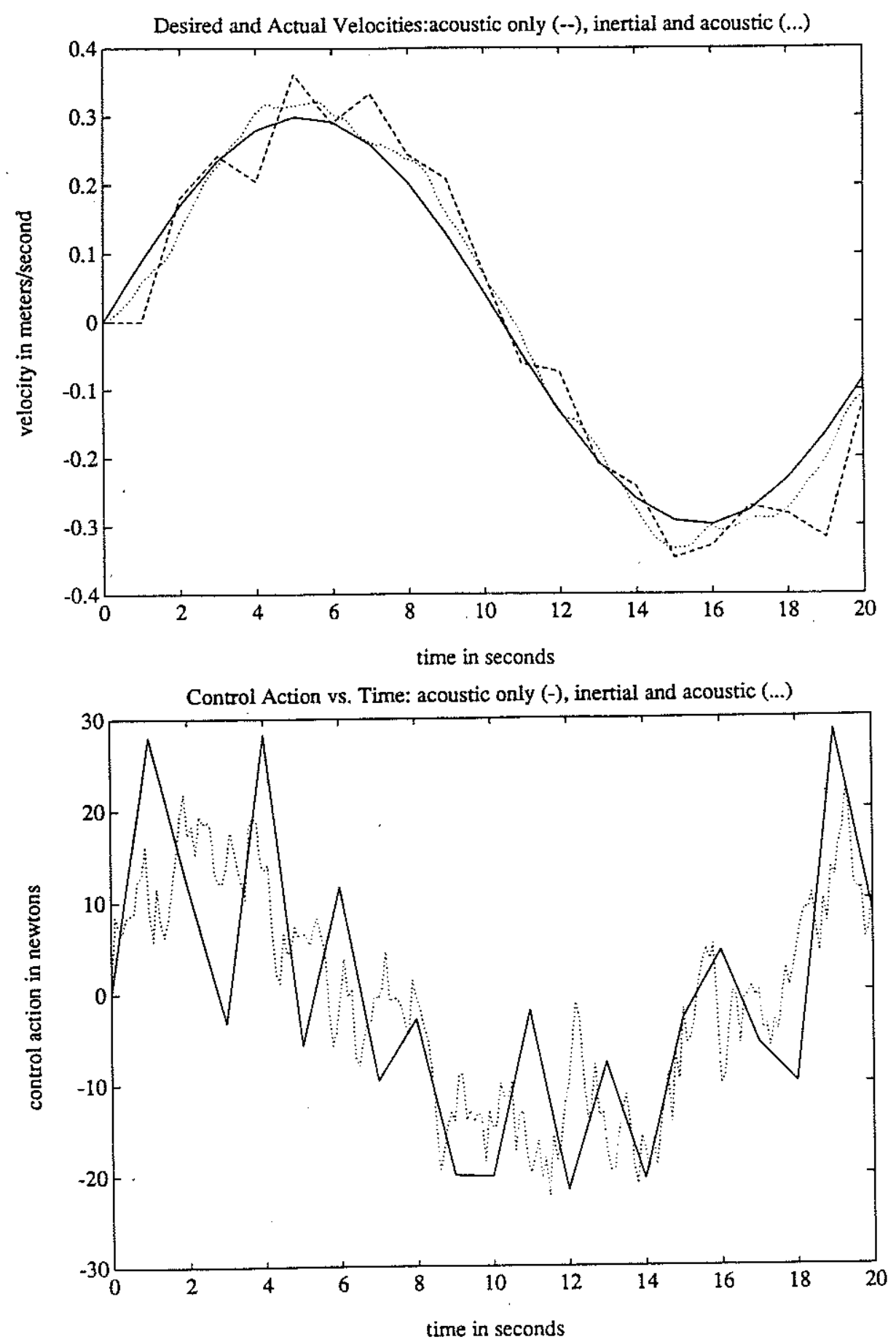

Figure 5-6: The interval between acoustic measurements was lengthened for this case to 1 second. The acoustic noise level was returned to .02 meters, however. The results show a fair improvement but unlike the dramatic comparison in Case 2. These two plots show the best contrast. 

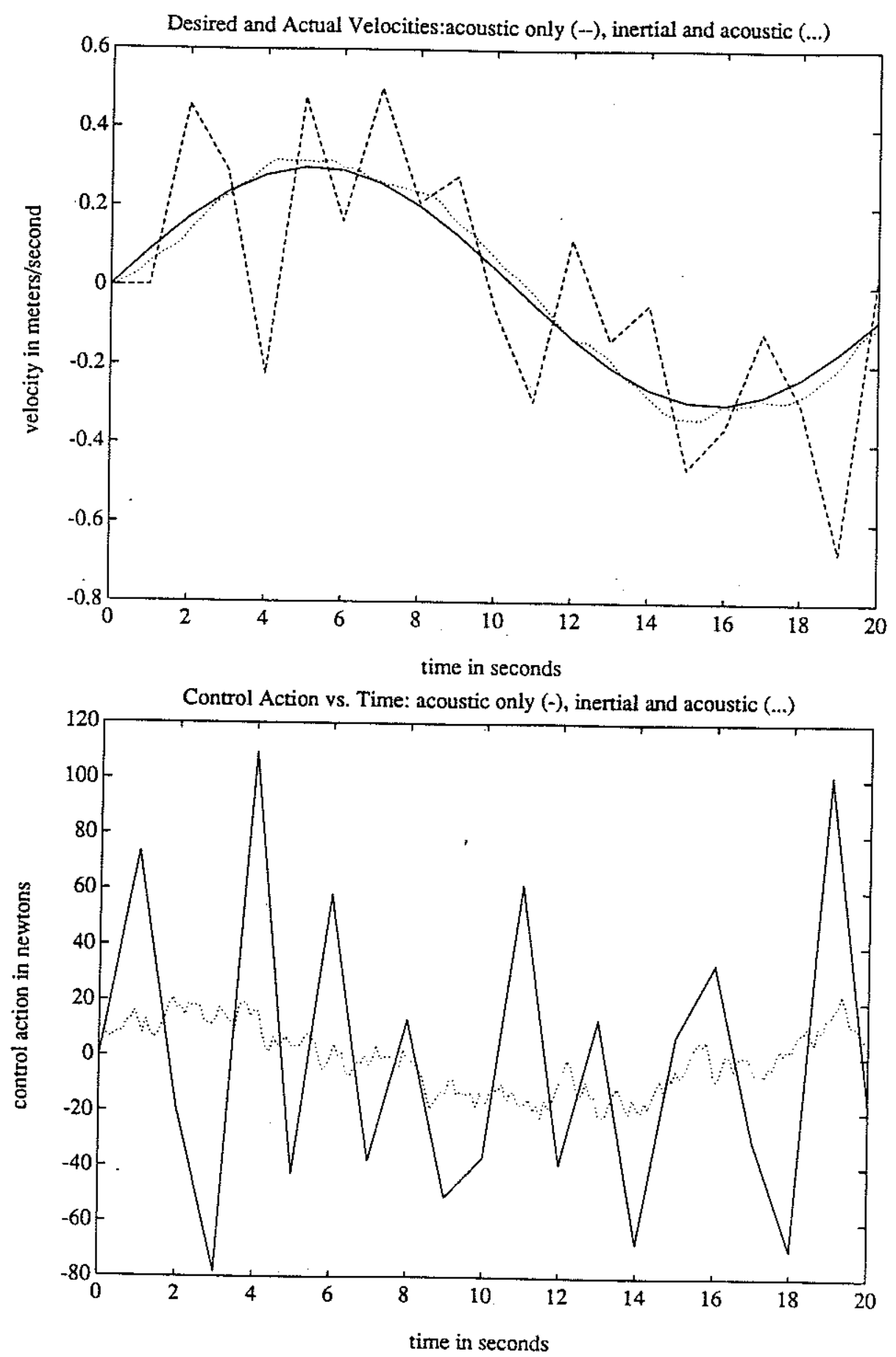

Figure 5-7: This last case is typical of a longer range, lower frequency, acoustic positioning system. The acoustic update rate is still $1 \mathrm{HZ}$, but the acoustic noise level has been elevated to .1 meter. As expected, This simulation shows the largest improvement of the four when the system is inertially augmented. 


\section{Chapter 6}

\section{Hybrid State Estimator for ROV JASON}

This chapter discusses the implementation of a hybrid state estimator for ROV JASON in the operational scenario presented in the first section.

\subsection{Operational Scenario}

Using an oceanographic research vessel as a mother ship; launch, control and track a remotely operated vehicle deployed beneath the vessel. Maintain the ROV on a track specified in real world coordinates by using a closed loop control system which depends on estimates of position and velocity provided by a two stage Kalman filter. The two stage filter is required because there are two components to the real world position equation. Each component has different noise characteristics and update rates which necessitates two stages in the filter. The trajectories required of the ROV are benign enough that the ability of the mother ship to maintain the ROV within the range of the acoustic net is not an issue. Manual control of the ship's steering and propulsion is anticipated during the trajectory following exercises. While the position information is to be used for closed loop control, measurement of the position of the vehicle with the greatest accuracy is the highest priority. Figure (6-1) shows the envisioned cooperation among the various equipments as described above. 


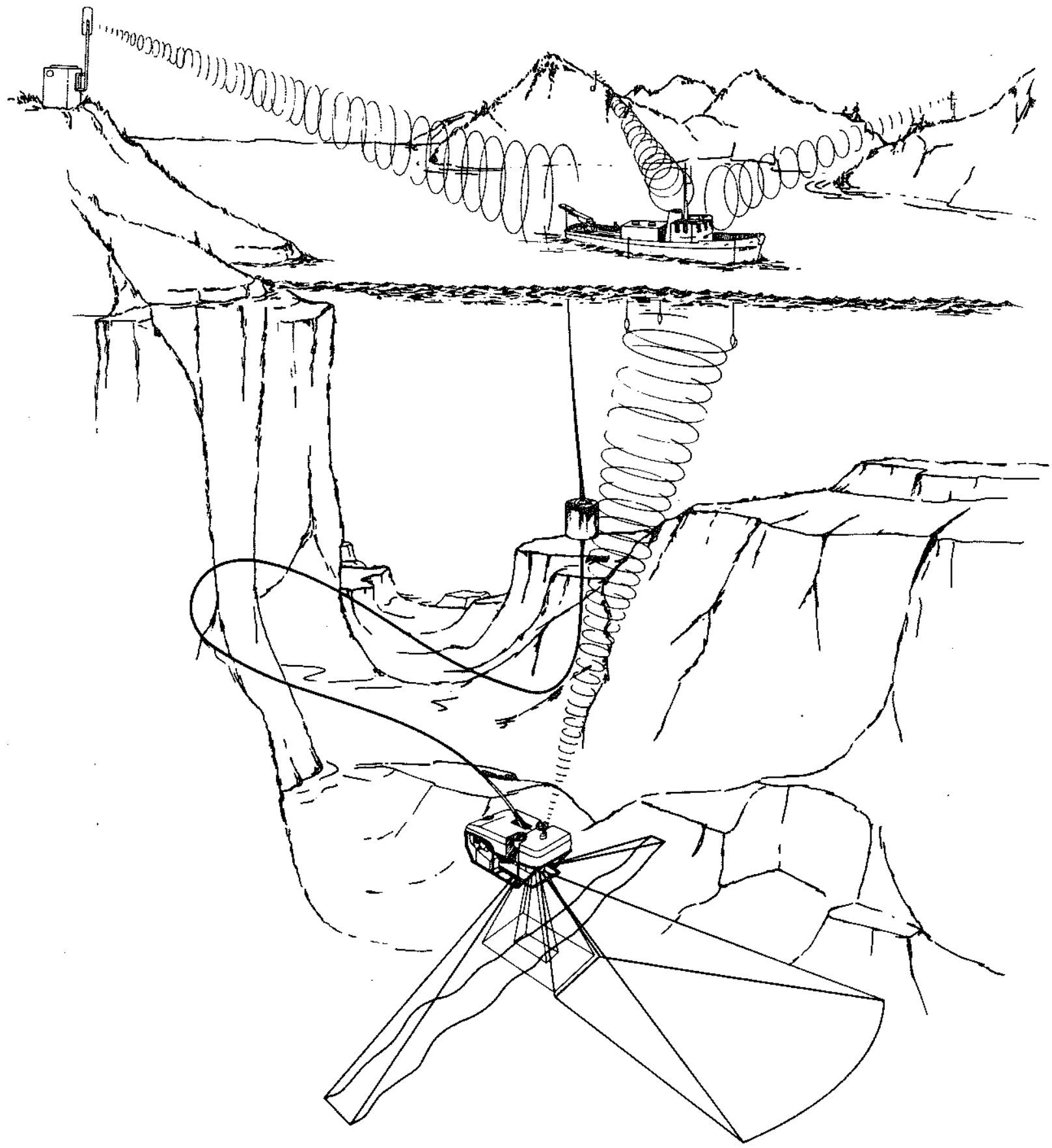

Figure 6-1: An envisioned tracking system integration using both acoustic and RF positioning systems. This arrangement results in a mobile, high frequency, high accuracy, acoustic tracking system for closed loop control of ROV's. 


\subsection{State Estimation for JASON}

\subsubsection{Role of the State Estimator}

As mentioned in Chapter 1, very precise underwater position tracking has been demonstrated using a high frequency, long baseline, acoustic transducer net. The implementation of such a system, however, has generally been confined to extremely small areas, with fixed transducer nets. Yoerger [3], for example, conducted his experiments with the $R P V$ in a test pool. A need exists to bring this type of precision to operations over a much larger geographic scale. The implementation explored in this chapter is the mounting of a system such as SHARPS, on a moving platform (research vessel) and operate in the manner described in the preceding Operational Scenario. The position of the transducers which make up the net would be fixed relative to the platform.

If information on the ROV relative to the moving net can be combined with the (time dependent) real world coordinates and the orientation of the net itself, high precision tracking can be extended to large areas of interest.

These two sources of information are best combined by a state estimator.

\subsubsection{Objectives of the State Estimator}

The objective of state estimation in this implementation is to increase the operational range without allowing accuracy to suffer significantly as a result of higher noise levels. As indicated previously, the noise in the RF position measurements is an order of magnitude higher than the noise in the acoustic positioning system.

Ordinarily, a Kalman filter approach might permit us to obtain a noise level in the position estimate which is smaller than either of the two individual levels. In our case, however, the final position is a linear combination of the two measurements and would suggest that the noise is simply the sum of the two separate levels. A hopeful feature of the physical system is that the dynamics associated with the ship are much slower than those of the ROV. It is hoped that we can take advantage of this feature and reduce the effect of the noise. 


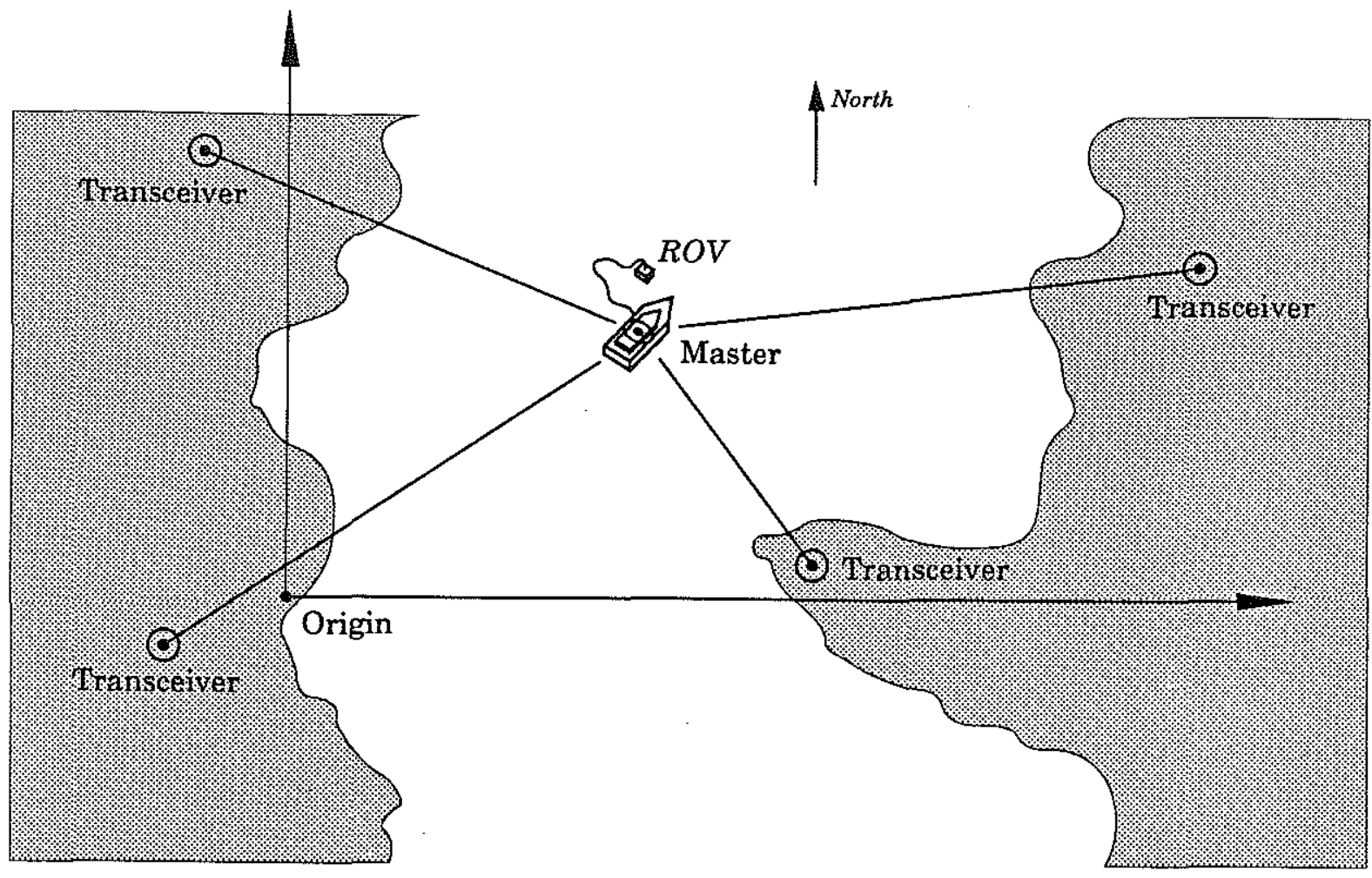

Figure 6-2: A typical arrangement of an RF positioning system for use with a research vessel in confined waters. The master transceiver is located on the vessel with the land based transponders slaved to it.

\subsection{Available State Measurements}

Two position measurements are available, but they are both required to obtain the geographic position of the ROV. The first is the position of the surface platform relative to an RF navigation net, and the second is the position of JASON relative to the high frequency acoustic net. The specifics associated with these measurements are discussed in the following paragraphs.

\subsubsection{RF Navigation System Measurements}

Tracking of the ship and hence the acoustic net is performed by an RF positioning system. This system uses a master transceiver on the ship's mast and a set of three or four slave transmitters (transponders) strategically placed in the vicinity of the ship's operations. Figure (6-2) shows a two dimensional view of the typical system setup. 
The system contemplated for use is manufactured by DEL-NORTE. It has an advertised update rate for individual ranges of approximately $10 \mathrm{HZ}$ at a range of up to several kilometers. The advertised maximum range of the system is 80 kilometers. Because most installations have involved customized computer interfacing and processing algorithms, the range update rate can be somewhat faster or slower than 10HZ. Typically, position update rate is no greater than about $5 \mathrm{HZ}$.

The accuracy of the system, if properly calibrated, is advertised as \pm 1 meter for position measurements. Available resolution from the system is 0.1 meters. Actual range measurements using individual transponders exhibited a reasonably gaussian distribution with a standard deviation of .2 meters at a range of 7 kilometers. (One of three transponders tested, however, exhibited a slightly skewed measurement noise distribution with a standard deviation of .3 meters.)

Extrapolating from range accuracy to position accuracy requires consideration of additional variables. Angular separation of the transponders as seen by the platform and the mechanics of the position fixing algorithm are two of the most important factors in making the extrapolation. Angular separation is principally a function of operator selection (within the constraints of the geography), while the details of the position fixing algorithm are proprietary. It is known that a synchronization pulse permits multiple ranges to be de-skewed to the time of actual initiation of the update sequence. When more than two transponders are in operation, the system calculates fixes based on all of the permutations of available transponders and produces a single fix whose cartesian errors are much smaller than that predicted by the range errors of individual transponders.

\subsubsection{Mobile Acoustic Positioning System}

The high frequency acoustic positioning system under discussion here is the same one that was covered in earlier chapters. In this application, the acoustic transponder net will be mounted in positions fixed to the ship and the master transceiver will be mounted on the ROV. Clearly, the difference here will be that the acoustic net will move with the motions of the research vessel. The following paragraphs discuss some of the implications of mobile installations. 


\subsubsection{The Research Vessel}

The mother ship on which the acoustic net is to be mounted is 106 feet long, 26 feet wide and weighs 245 tons. It's propulsion capabilities consist of an $850 \mathrm{HP}$ main engine and a bow thruster which develops 3220 lbs of thrust, trainable through 360 degrees. Its draft is 9 feet and its main mast (at the yardarm) is 40 feet above the waterline. A profile of the vessel and a listing of the ship's salient features is included as Figure (6-3). The plan is to rigidly mount the acoustic transducers on poles (fixed to the sides of the vessel) which will extend below the keel of the ship. To attach the transducers to the poles, the transducers are wrapped in rubber and then wedged into the end of the hollow aluminum poles. This insures that the actual attachment points of the transducers are sound isolated.

While the ship is not particularly large, it is expected to be an extremely stable platform for the protected waters in which the experiments are to be conducted.

It becomes imperative in this application that the attitude of the research vessel be monitored and that the position measurements be compensated for the affects of the ship's attitude changes.

\subsection{Attitude Determination of the Research Vessel}

Conducting experiments in sheltered inland waters, significant changes in the support ship's attitude are not expected. This is not to say that attitude information is not important in determining the position of the ROV in a consistent, cartesian, frame of reference. The accuracies desired for SHARPS and to a lesser degree, DEL-NORTE, require attitude compensation.

Since pitch and roll have a restoring force created when the ship changes attitude, this produces oscillatory motion. Depending on the nature of the seas and the wind oscillatory motion can develop in yaw also. Yaw (heading) is also a desired result of operator input to the rudder and propulsion.

\subsubsection{Pitch and Roll Effects}

There are two basic effects from pitch and roll. The first effect is the change in position of the RF Master transceiver relative to the RF net and the second is the change in position of 


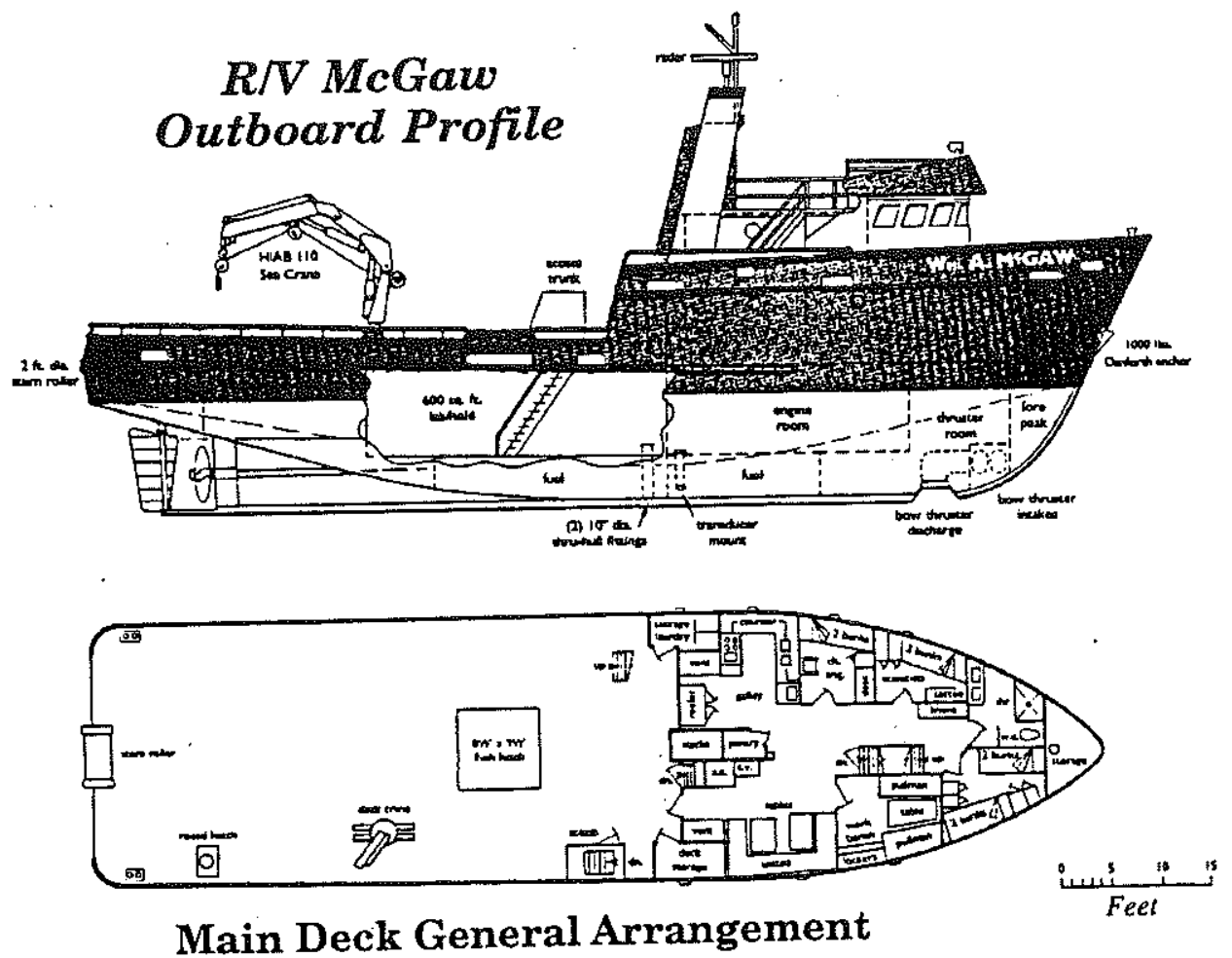

Machinery:

- Main Engine:

CATD398TA, $850 \mathrm{HP}$ @ $1225 \mathrm{RPM}$ with 4.22 to 1 reverse/ reduction gear

- Bow Thruster: WHITEGILL $222 \mathrm{HP}$ (a) $1000 \mathrm{RPM}$ 3220 lbs. thrust, pump type unit, 360 trainable, flush mounted
Characteristics:

- Length: $106 \mathrm{ft} ., 3$ in.

- Beam: $26 \mathrm{ft}$.

- Depth: $13 \mathrm{ft} ., 4$ in.

- Draft: $9 \mathrm{ft}$.

- Work deck: $25 \mathrm{ft} . \mathrm{X} 50 \mathrm{ft}$.

- Gross tons: 245

- Speed: 11 knots +

- Range: $\quad 5,000 \mathrm{~nm}$

- Endurance: 21 days

- Fuel efficiency: $35 \mathrm{gals} / \mathrm{hr}$. @ 10 knots

Figure 6-3: The R/V McGaw is the vessel that has been selected for the integrated RF and acoustic positioning system experiments with JASON. The RF master transceiver will mount on the mast and the SHARPS acoustic net transceivers will mount on the underside of the vessel. 


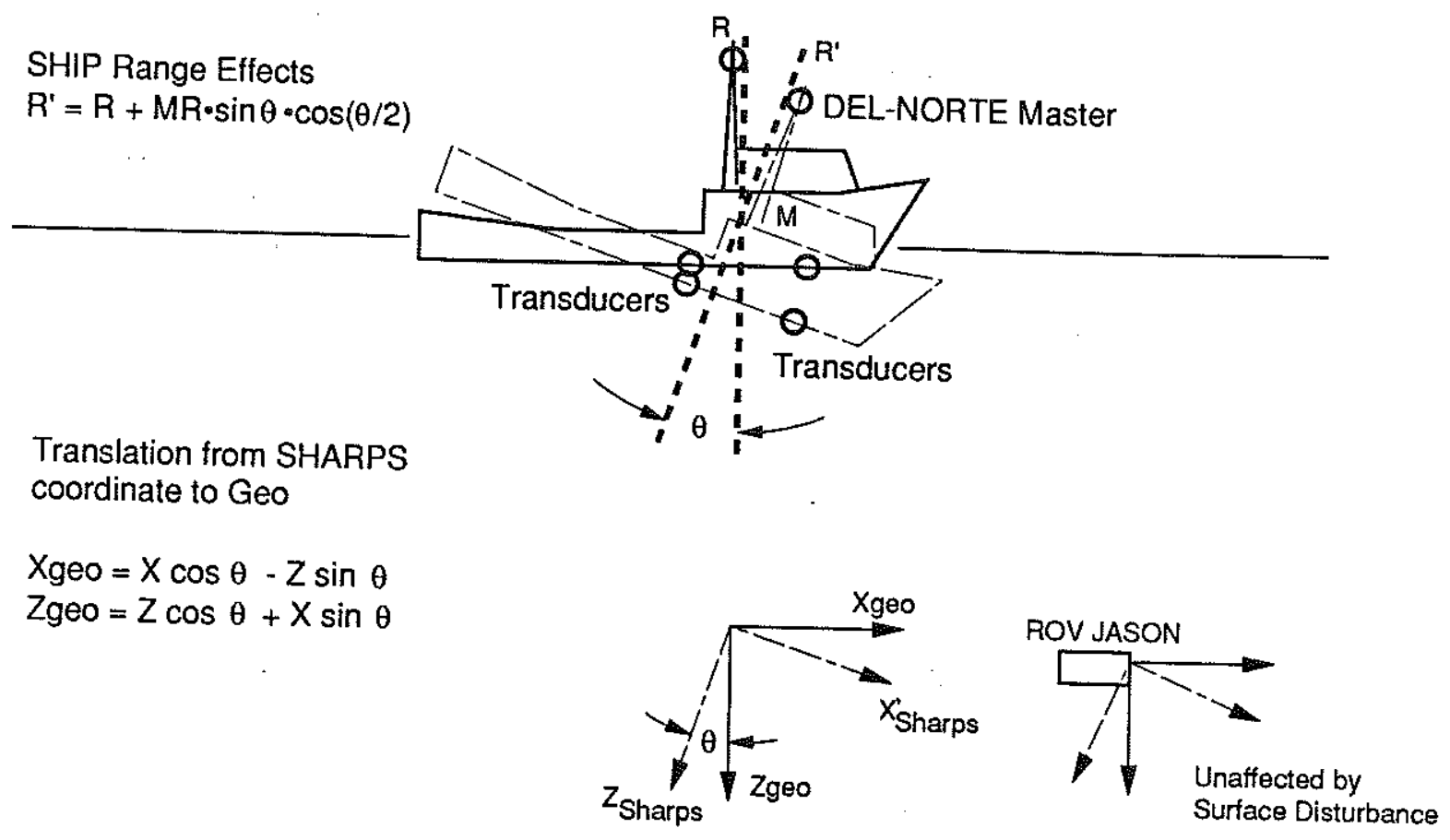

Figure 6-4: Geometric distortions resulting from pitching of the support ship. Note that the pitch affects both the position of the RF Master Transceiver and of the acoustic net, but in opposite directions.

the acoustic net with respect to the ROV. For very small angles, if we use the small angle approximation we can treat the effects of pitch and roll separately. The sketches in figures (6-4) and (6-5) illustrate the attitude effects on the position measurements.

\subsubsection{Attitude Sensors}

The sensors required to monitor the research vessel's attitude are similar to those in the SIMS package on JASON JUNIOR. Because of the much slower dynamics and smaller angles expected of the vessel there are a smaller number of sensors required. The sensors to be used are:

- A Wastson Inclinometer mounted with its sensitive axis aligned with the ship's pitch axis.

- A second Watson unit mounted with its sensitive axis aligned with the ship's roll axis. 


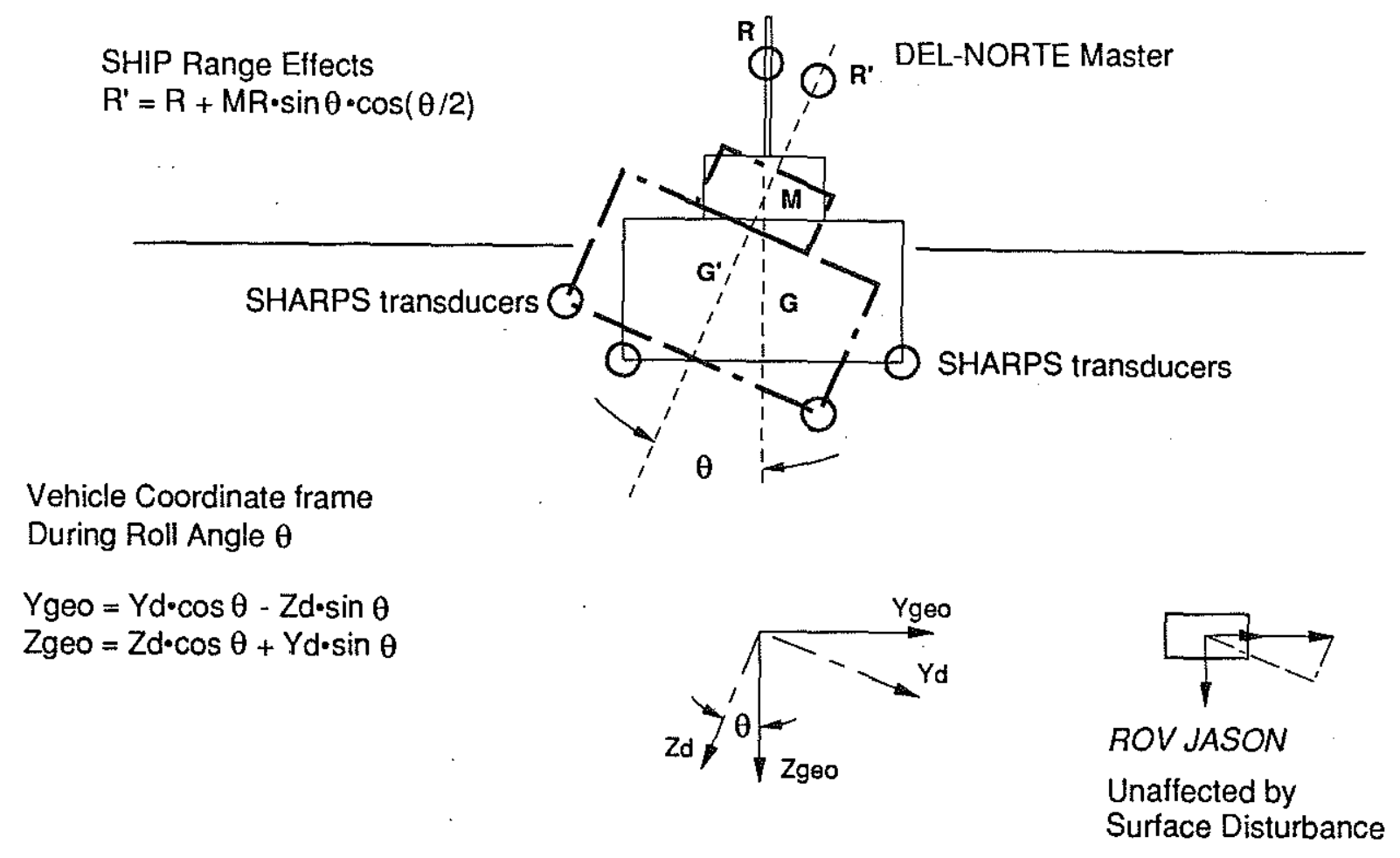

Figure 6-5: Geometric distortions resulting from rolling of the support ship. Note that roll also affects both the position of the RF Master Transceiver and of the acoustic net.

- An ENDECO Fluxgate compass, also described in Chapter 4.

The Watson inclinometers use the combination of a pendulum for static angular measurement and an integrating rate sensor to provide dynamic measurements. Figure (6-6) shows the typical frequency response for this inclinometer.

\subsubsection{Vessel Attitude Calculation}

The calculation of the ship's attitude is relatively straightforward. Again we refer to the Euler angle representation presented in Chapter 4 for assistance.

Because the compass is gimballed, it gives us a true Euler angle for heading. The roll and pitch inclinometers, however, give us angles which need to be resolved into the appropriate euler angles. After the true euler angles are determined, they are transformed into real world coordinates using the coordinate transformation matrix developed in Chapter 4. 


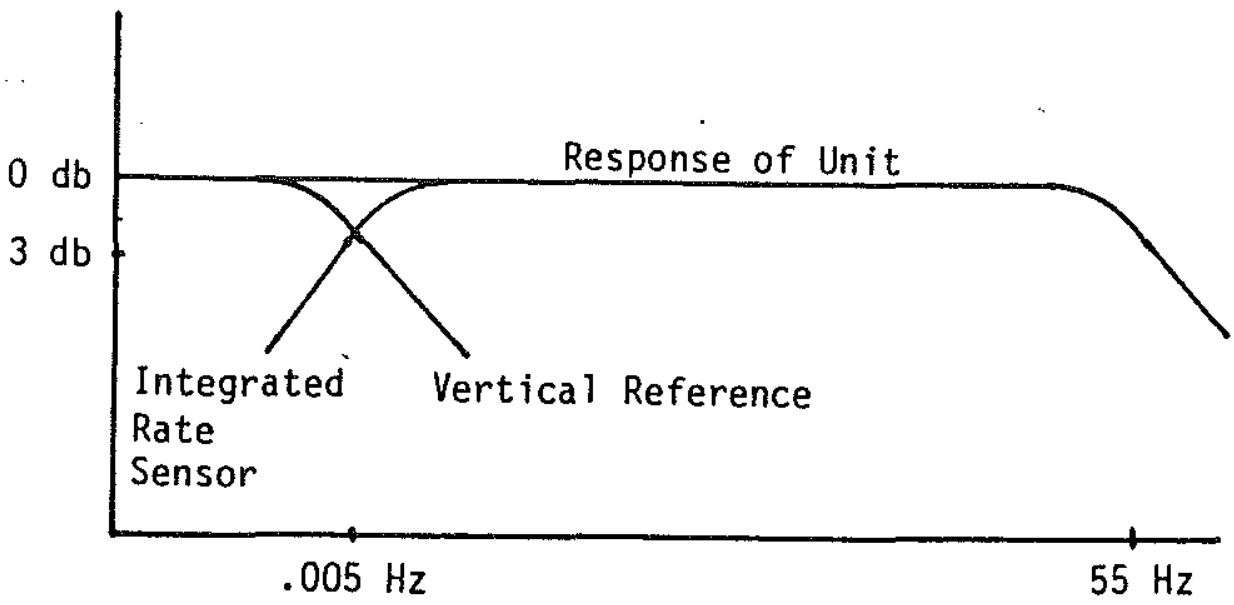

Figure 6-6: The frequency response plotted for the static and dynamic angular sensors in the Watson Inclinometer. This unit replaces the separate inclinometer and angular rate sensor employed on JASON JUNIOR'S attitude package.

\subsection{Two Stage Kalman Filter Development}

Because the noise distributions observed in measurements with both RF and acoustic positioning systems are basically zero mean and gaussian, and because the dynamics of the two systems (ship and ROV) can be reasonably represented by linearized models, a Kalman filter approach to the synthesis of the measurements is proposed. We will use the Kalman filter gains in an estimator of SHIP and ROV position. The estimates of position can then be used in the control law for control of the ROV.

Because we know the dynamics are actually nonlinear, however, we know that we will not have the optimum estimator. What we hope to accomplish is to eliminate the effect of the lower quality RF position information and provide for a workable mobile high frequency acoustic navigation system.

We first start with the one-dimensional case of combining two range measurements. Be- 
cause the two measurements will not be available at the same instant, a two-stage filter approach is necessary.

Although the dynamics of the two vehicles are essentially uncoupled, the geographic position of the ROV is the sum of the two measurements being taken. I have made the assumption that the measurements are available sequentially with a .1 second delay between different measurements and with a .2 second delay between similar measurements.

Different timing schemes are necessary depending on the measurement update rates. If the measurements were available at different update rates, the same basic approach is taken, but additional complexity is required to account for the varying nature of the time between different measurements. For example, if the update rates were $5 \mathrm{HZ}$ and $2 \mathrm{HZ}$, then every second update of the $2 \mathrm{HZ}$ measurement would coincide with a $5 \mathrm{HZ}$ measurement and the $C$ matrix for the coincident measurements would be quite different than those for individual measurements. Also the amount of time over which to extrapolate estimates until the next measurement would rotate through $.1 \mathrm{sec}, .2 \mathrm{sec}, .3 \mathrm{sec}$, and $.4 \mathrm{sec}$ and would require the calculation of separate $\Phi$ and $\Gamma$ matrices for each time interval.

An alternate approach is to propagate estimates at .1sec intervals and then incorporate whichever measurement(s) corresponded to the particular interval. Figure (6-7) illustrates this latter approach.

\subsubsection{Kalman Filter Assumptions}

In the actual implementation of the state estimator, simplifying assumptions are needed to implement the multistage filter. Using these assumptions, we have tested the algorithms in a computer simulation.

Beyond the assumption that the dynamics are linear, the first assumption is that the measurement noises are all zero mean, have normal distributions, and are uncorrelated. This assumption, as illustrated in previous sections, is a good one. In general, an assumption that the measurement noise has a normal distribution is well supported by the Central Limit Theorem [21] because the measurement noise results from so many different sources.

The second assumption is that the driving noise is white noise. For our simulation we will assume that the driving noise is white and that this represents disturbances, fluctuations in 


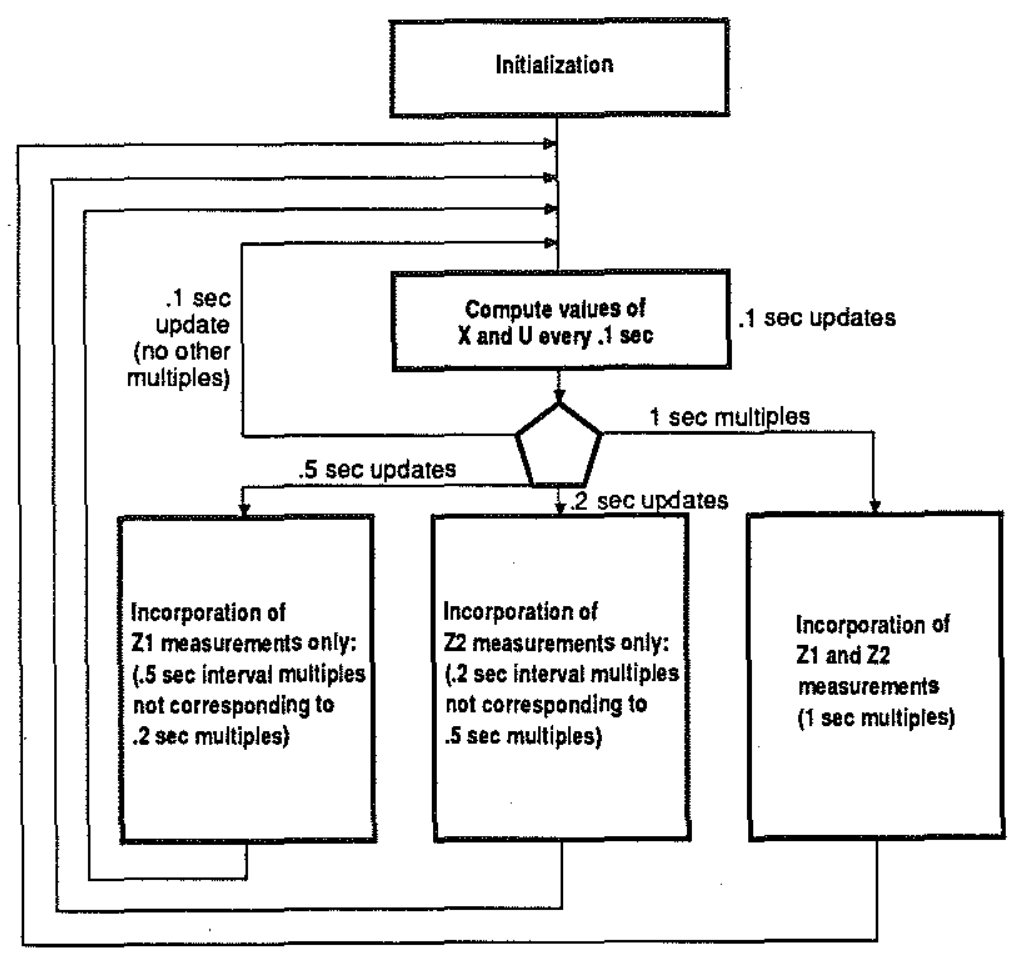

Figure 6-7: This block diagram illustrates the complex flowpath that results when measurements are taken at different times and with different frequencies. It is assumed in this example that the acoustic measurements are made every .2 seconds and the RF position measurements are made every .5 seconds. As a result, the measurements coincide on the even second, every fifth (second) measurement of acoustic (RF) position.

main engine or thruster output, etc. Here we have less evidence, but we rely on the Central Limit Theorem to prove this to be correct for our purposes.

The largest assumption we will make as applied to this simulation specifically is that all of the conditions are met to allow us to use a stationary Kalman Filter [13], [14]. In other words the following conditions apply to our simulation example:

- System and measurement dynamics are linear,

- System and measurement equations have constant coefficients (A, B, C, and G are not functions of time), and

- Driving noise statistics are stationary ( $Q$ and $R$ are not functions of time.)

Gelb et al also point out in Applied Optimal Estimation that the condition of 'complete 
observability' is sufficient to assure the existence of a steady state solution, while the condition of 'complete controllability' will assure that the solution is unique.

\subsubsection{Two-Stage Kalman Filter Design}

For the Kalman filters in this investigation, we use a discrete expression for the dynamics which is a function of the sampling interval chosen. Since both measurements have an update rate of $5 \mathrm{HZ}$, the sampling interval $(\mathrm{T}$, or $\mathrm{dt}$ ) is .2 seconds. To maximize the time delay between different sensor measurements, I have chosen to space the acoustic and RF position measurements .1 seconds apart. As a result of this timing, a need exists for two different sets of propagation equations to properly advance the estimate of the states of the vehicle. (Again the total distance is the addition of the two measurements.) For example:

Between each measurement of $X_{J a s o n}:(\Delta t=d t=.2 \mathrm{sec})$

$$
X_{J_{a s o n}}(k+1)=A * X_{J a s o n}(k)+B * U(k)
$$

Between measurement of $X_{J a s o n}$ and $X_{\text {Ship }}:(\Delta t=d t=.1 \mathrm{sec})$

$$
X_{\text {Jason }}(i+1)=A 1 * X_{J a s o n}(i)+B 1 * U(i)
$$

A, A1, B, and B1 are the appropriate Phi and Gamma matrices corresponding to $\Delta t$ 's of .1 second and .2 seconds.

For both cases:

$$
\begin{aligned}
& y(k)=C * X_{S h i p}(k) \\
& y(i)=C * X_{J_{a s o n}}(i)
\end{aligned}
$$

The actual states are generated from the following equations and produced every .1 seconds:

$$
\begin{array}{r}
X(i+1)=A 1 * X(i)+B 1 * U(i)+W(i) \\
y(i)=C * X(i)
\end{array}
$$

Where $W(i)$ is the white driving noise. 


\subsection{Simulation Design}

In order to test the efficacy of the filter designed in the previous section, I developed a simulation in the PC MATLAB programming language. The code has three sections; initialization, simulation loop, and plots/analysis.

The initialization section simply initializes all of the variables and allows me to introduce any initial estimate error, tailor the control action for the SHIP and the ROV, and set the length of the simulation run.

The simulation section has two loops inside the overall loop. Only one of the inner loops is completed for each transit of the outer loop. This is because actual state and control action information is computed for each .1sec interval, while the loop for a specific measurement, estimate cycle occurs only every .2 seconds. Since the dynamics of the SHIP and the ROV are not coupled, measurements of the distance from the SHIP to the ROV are accomplished by subtracting the actual position of the ship from the actual position of the ROV at that instant and adding random noise characteristic of the measurement system to it. The following equation expresses these relationships:

$$
\begin{array}{r}
Z_{\text {ship }}(k)=X_{\text {ship }}(i)+v 1 * \operatorname{rand}(1) \\
Z_{\text {jason }}(k)=\left(X_{\text {jason }}(i)-X_{\text {ship }}(i)\right)+v 2 * \operatorname{rand}(1)
\end{array}
$$

Note that there are mixed subscripts in the above equations. This is how I reflect that a particular measurement only occurs every .2 seconds, while the actual values are simulated every .1 seconds.

Several simulations were run and are listed in Table (6.1).

Since the inputs were all initialized as zero, switching between an input and no input was accomplished by commenting out or in the appropriate input description in the .1sec simulation update loop.

The PC MATLAB code is very readable' and is heavily commented. It is included as an appendix. There are three separate 'programs'. The first two calculate the steady state Kalman filter gains and the third one is the simulation code described above. (An additional program is used for more extensive graphic analysis of the data.) 


\begin{tabular}{|c|l|}
\hline \hline \multicolumn{1}{|c|}{ Description of Input } \\
\hline 1. & No Inputs, only driving noise to SHIP and ROV \\
2. & Inputs to ROV only, but not modeled in observer meters \\
3. & Input to SHIP only, but not modeled in observer \\
4. & Inputs to SHIP and ROV, not modeled \\
5. & Inputs to both and both modeled \\
6. & Inputs to both, PLUS initial SHIP position error \\
7. & Inputs to both, PLUS initial ROV position error \\
\hline \hline
\end{tabular}

Table 6.1: Different combinations of modeled and unmodeled inputs used in the simulations.

Graphs of estimated, measured and actual SHIP position as well as estimated, measured and actual ROV position are included in this section. There are six graphs total; two from the two input/both modeled case (No.5) and two associated with each initial error runs (No.6 and No.7).

In looking at the graphs, it is no accident that the actual state values and measurements are the same when the inputs are identical. The seed for the random number generator is reset to the same value for each simulation run. In the event, however, these estimates were used in a control loop where $U(k)$ was calculated based on the estimated states, then the actual state values might vary considerably.

\subsection{Two Stage Kalman Filter Tracking Simulation Results}

This section includes the results of the simulations described in the section above. The analysis of the simulations was performed graphically, by means of error plots and numerically, by taking the standard deviation of the error values over the period of the simulation.

The period of the simulations was chosen to be 10 seconds, for a total of approximately 100 actual positions, 50 SHIP position measurements, 50 ROV measurements, and 100 geographic ROV position estimates. This length of time was chosen for several reasons. Longer runs resulted in essentially the same results but took much more processing time. Longer runs also tended to cause the position graphs to have compressed scales to fit all of the data, making it more difficult to grasp the significant features.

The plots of the errors corresponding to the position comparison plots included in the 

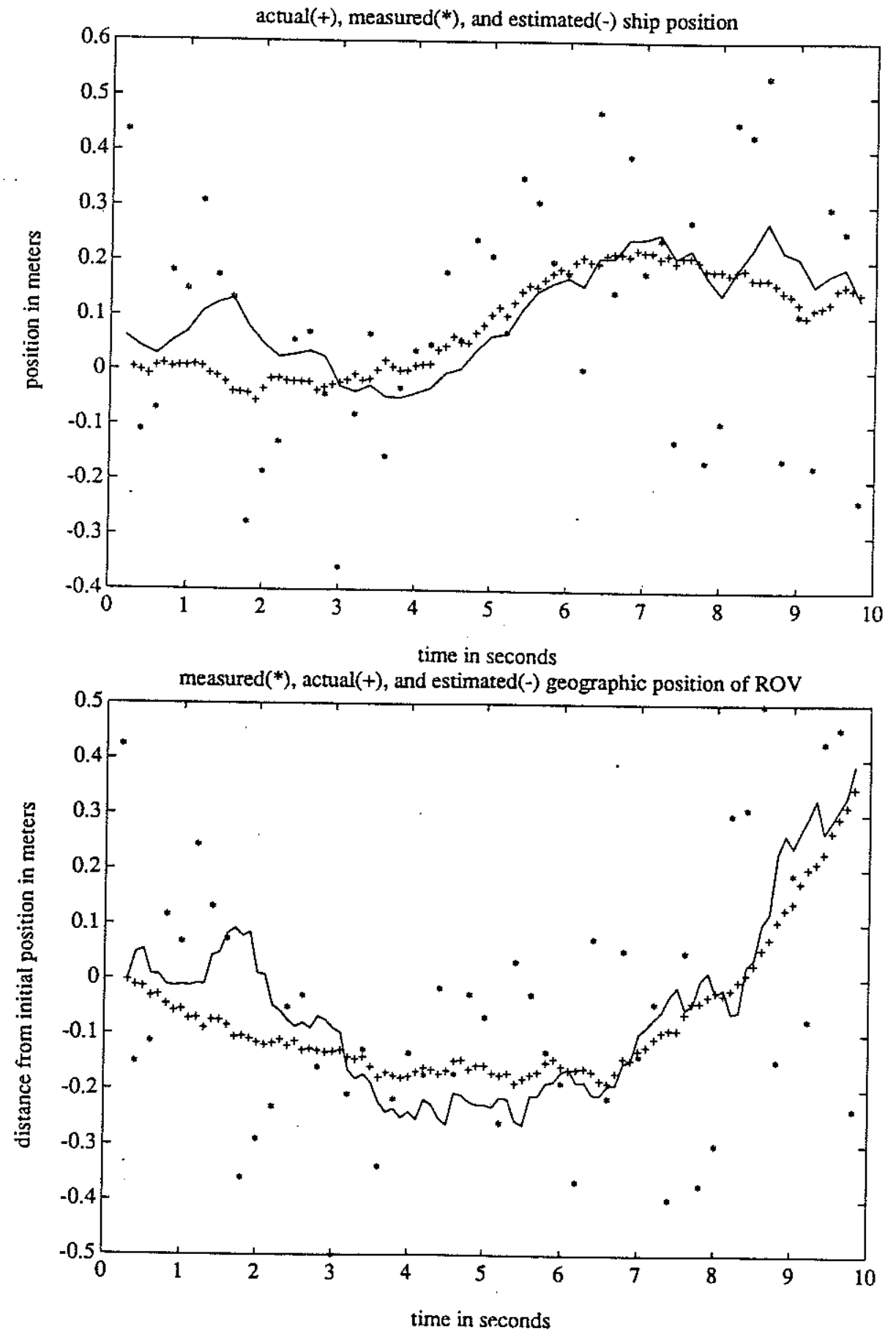

Figure 6-8: These simulation results offer a comparison between measured, estimated and actual position of the ship when both entities have control inputs and are modeled. The upper graph is for just the SHIP, while the lower graph uses the SHIP (RF) and the ROV (SHARPS) to get true geographic position. 

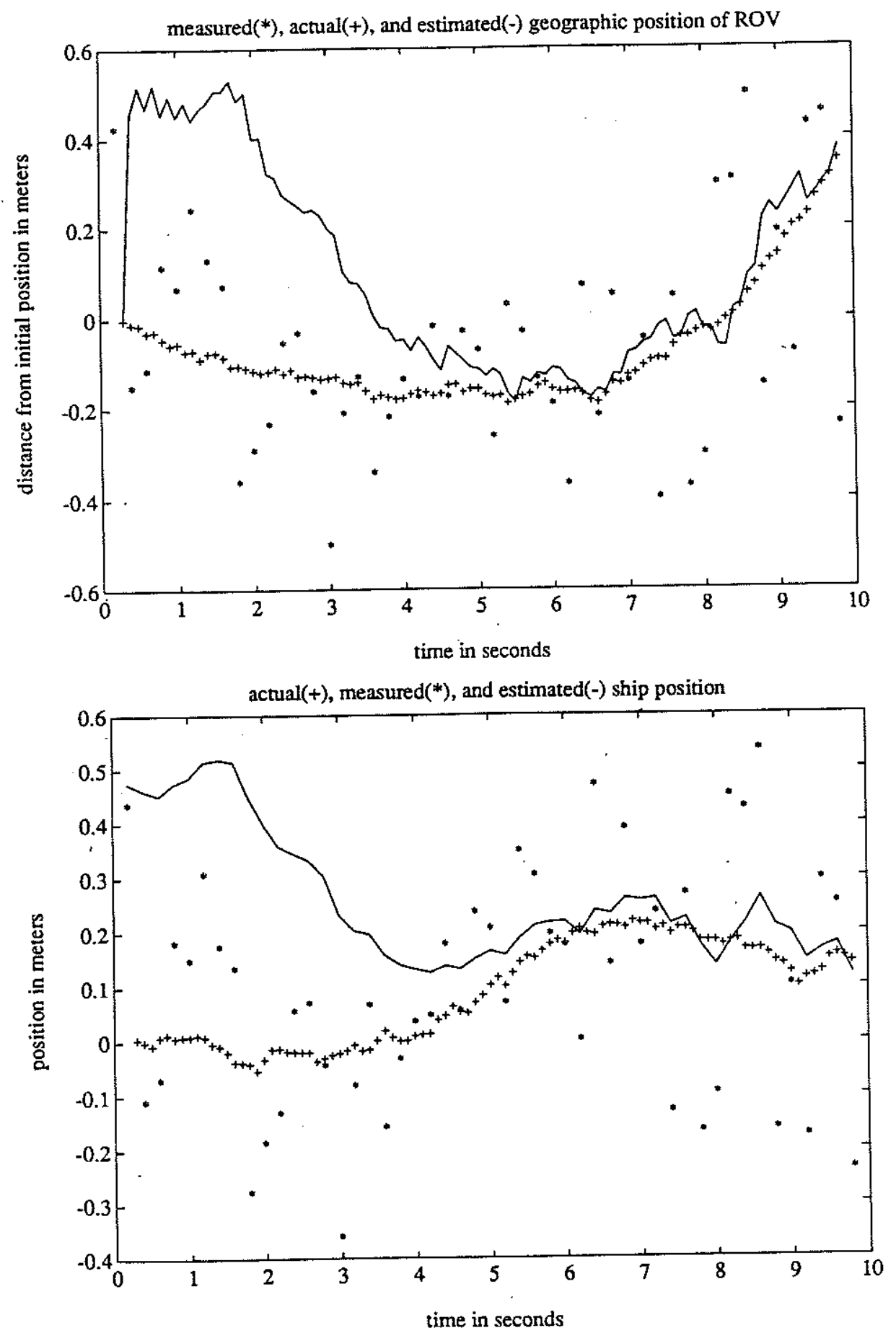

Figure 6-9: These graphs differ from the previous page because an initial SHIP position error of .45 meters has been introduced. As expected, both estimates are affected by the error, since the ROV uses the SHIP measurement as well. 

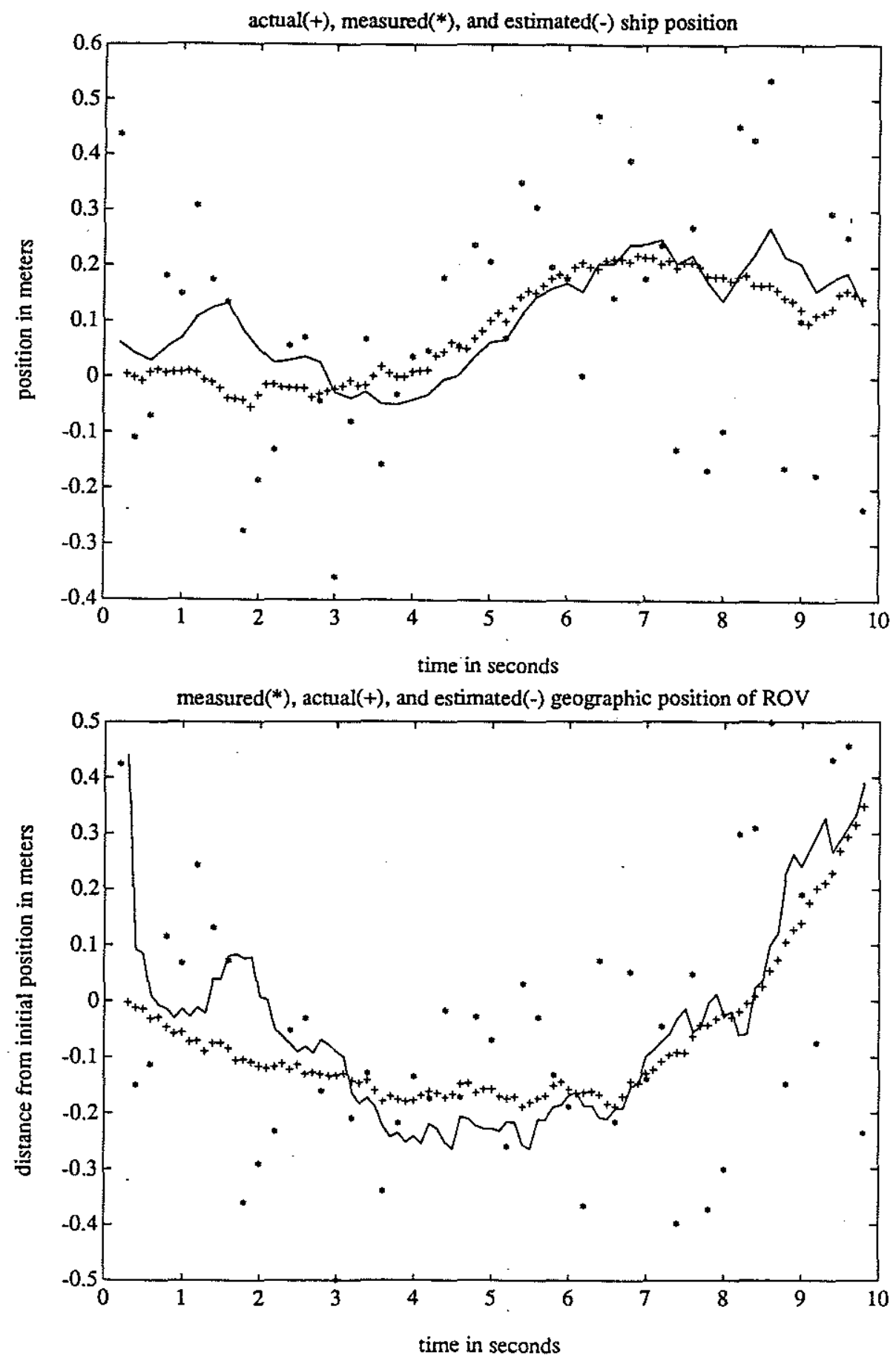

Figure 6-10: These simulation results show measured, estimated and actual positions when an initial ROV position error of .45 meters is imposed. Notice how much faster the estimated ROV position converges on the actual position in the lower graph. The SHIP position remains unaffected by the imposed error. 


\begin{tabular}{|l|l|r|}
\hline \hline \multicolumn{2}{|c|}{ Description of Input } & Standard Deviation \\
\hline 1. & Only driving noise & .0661 meters \\
2. & ROV input, not modeled & .0662 meters \\
3. & Ship input, not modeled & .0663 meters \\
4. & SHIP and ROV, not modeled & .0663 meters \\
5. & SHIP and ROV, both modeled & .0660 meters \\
6. & Initial SHIP position error & .8905 meters \\
7. & Initial ROV position error & .3439 meters \\
\hline
\end{tabular}

Table 6.2: Summary of Simulation Results.

\begin{tabular}{|c|c|c|}
\hline \multicolumn{2}{|r|}{ Description of Input } & Standard Deviation \\
\hline 8. & Extended simulation, SHIP IC error & .5728 meters \\
\hline 9. & No. 8 data excluding first 40 time steps & .1663 meters \\
\hline
\end{tabular}

Table 6.3: Results of Additional Simulation Runs.

previous section are presented in the following pages.

In the final analysis, the most important results are the errors in ROV geographic position. If the error can be made small enough then we can use it to permit closed loop control of an ROV using geographic coordinates instead of local ones. The logical extension to coordinate position estimation is ROV control based on the specification of desired trajectories in geographic coordinates. Table (6.2) summarizes the standard deviations of the errors in ROV geographic position.

Because there was such a marked jump in the standard deviation computed for the initial position errors, some additional runs were conducted. First, I lengthened the period of the simulation to 200 time steps, then I chopped off the first 40 time steps to see if the original standard deviation value would be recovered. These results are presented in Table (6.3)

These results alerted me to some erroneously favorable data contained in the first three .1sec intervals when all of the values were still zero. After excluding the offending points, however, insignificant overall differences in the results were seen. 


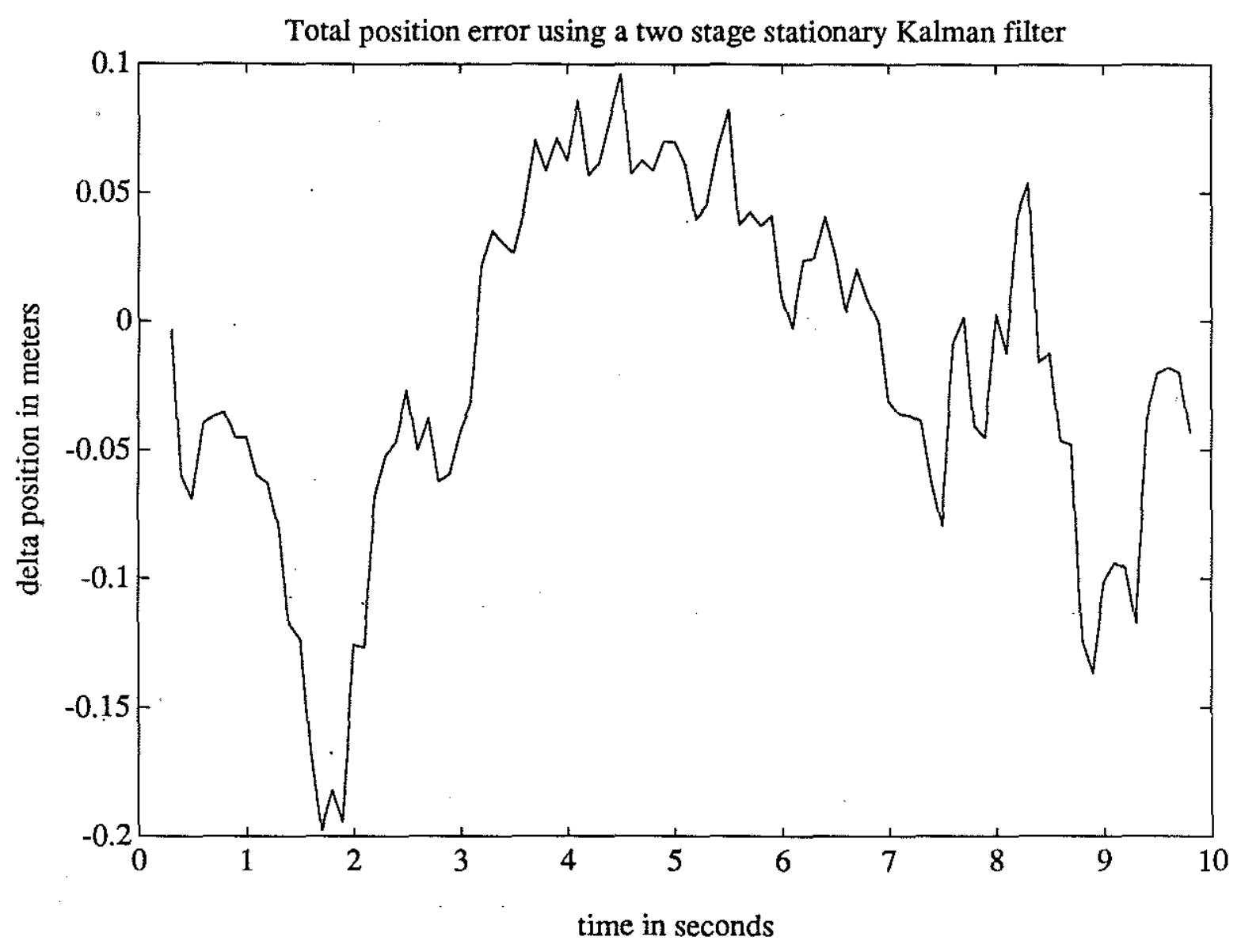

Figure 6-11: This error plot offers a measure of the difference between actual and estimated geographic position of the ROV. Remember that ROV position is a combination of the RF measurement of the SHIP and the acoustic measurement of the ROV relative to the SHIP. This means that the error is a linear combination of the individual estimation errors. 


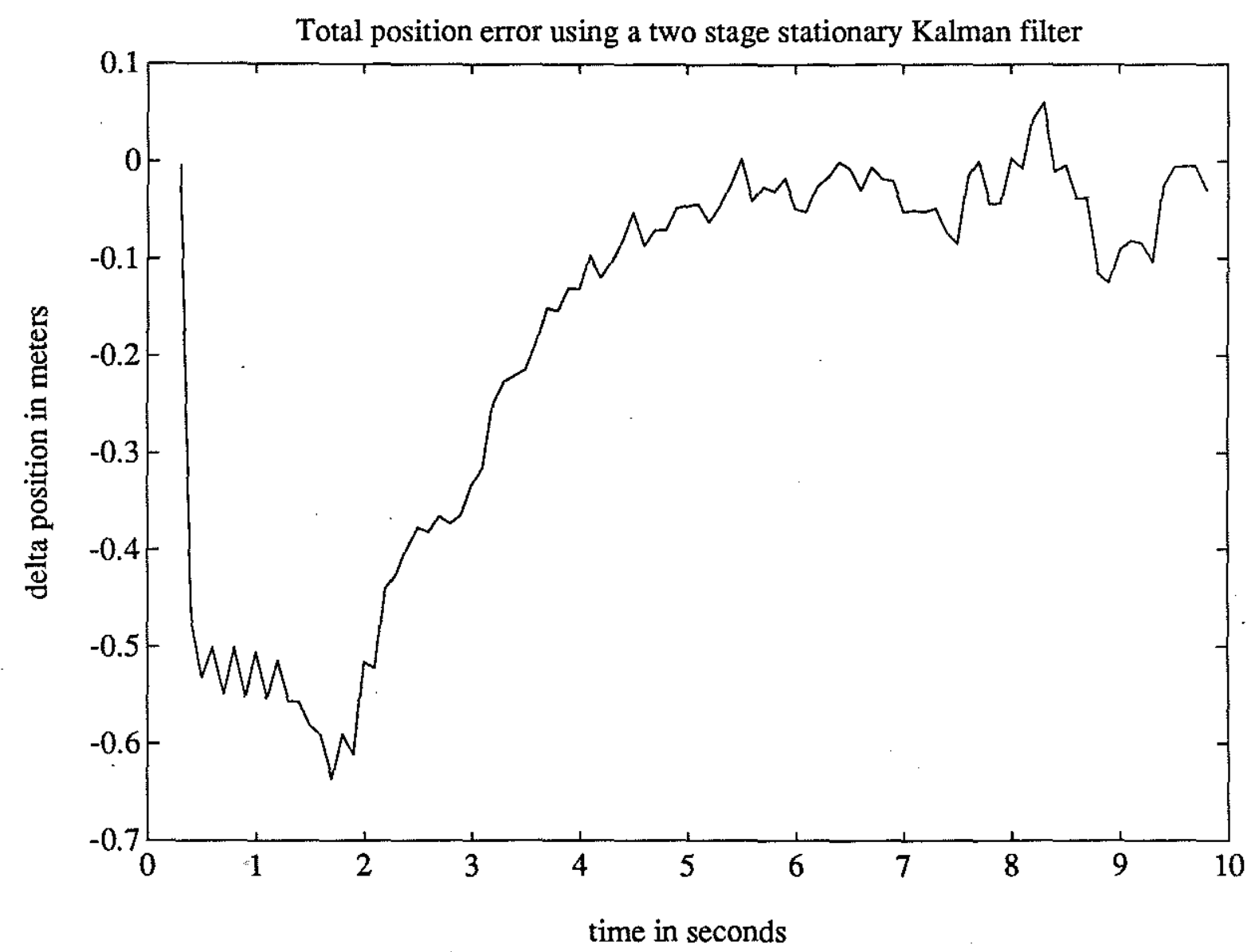

Figure 6-12: This error plot reflects the resulting total ROV geographic position error when a SHIP position error of .45 meters has been introduced. As expected, this initial condition shows up in the ROV geographic position error and gradually decreases as time passes. 


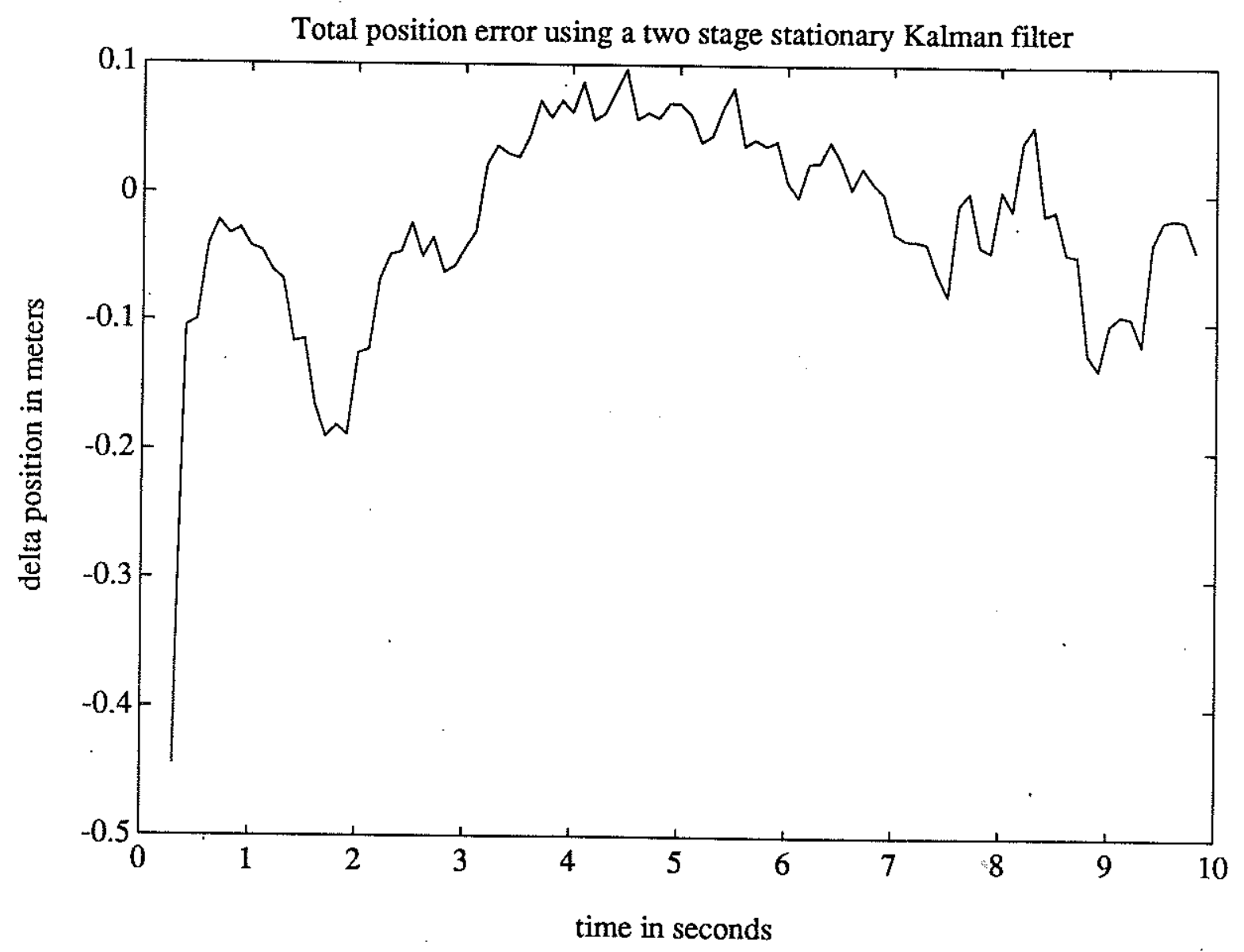

Figure 6-13: This plot illustrates the error between actual and estimated total geographic ROV position when an initial ROV acoustic position error of .45 meters is imposed. Notice how much faster this error tends toward zero than the error in the previous graph. This error is expected to be diminished faster than the SHIP initial position error because the new ROV acoustic measurements are weighted more heavily as a result of their higher accuracy. 


\subsection{Conclusions}

From the data, it appears clear that in the most straightforward case of one-dimensional range synthesis, Kalman filtering can reduce the error associated with random noise and disturbances significantly. There have been some significant assumptions made, however.

As we noted in the development of the Kalman filter, the assumption that the dynamics are linear is a poor one overall. The approach has been to linearize about the expected operating point and hope to draw some reasonable conclusions. Because both the linearized system and the nonlinear model are both stable, the effects of our linearization should not be too drastic. What it means, however, is that the filter designed may not be the optimum.

$I$ found it curious that all of the propagation modeling differences (except the initial condition errors) yielded standard deviations of the position estimate error which were very close together. My explanation for this phenomenon is that the simulated system inputs were nearly of the same order as the white driving noise levels. It is also interesting to note that the ROV input produced the largest effect when it was not modeled.

The initial condition error simulation runs produced interesting plots. An important conclusion is that the most damaging place for the error to occur is in the SHIP initial position estimate. This error propagates directly to the ROV geographic position estimate and is relatively slow to converge on the actual values of ROV and SHIP position.

An initial condition error in the ROV position is overcome more quickly as illustrated in the plots. I conclude that this is because more weight is placed on the acoustic measurements because they are much more accurate than the RF ship position measurements.

The 'hybrid' state estimators, using the stationary Kalman filter gain matrices, obtained results which were very similar for all of the different conditions displayed in Table (6.1). While this was not a time varying filter, we would have expected the same results given a small amount of 'settling time.' Because we were using a large number of measurements while simulating reasonable platform ship and ROV dynamics we expect convergence to the steady state filter and similar results among the different runs. 


\section{Chapter 7}

\section{Conclusions}

\subsection{Achievements Versus Objectives}

Our ultimate objective in both endeavors was to raise the control bandwidth for long range operations. For JASON JUNIOR we attempted to increase its control bandwidth by augmenting acoustic position measurements with inertial ones. For JASON, we approached the problem in relatively shallow, protected waters by mobilizing the acoustic positioning system on a powered platform and then tracking the platform itself. How did we do? Was the result worth the effort? What improvements can be made? The purpose of this chapter is to try to answer these questions.

\subsection{Control Bandwidth Improvements}

\subsubsection{Bandwidth Effects on JASON JUNIOR}

Although we technically have difficulty talking about the bandwidth of a system which is other than first order, there are several metrics which characterize a system's dynamic response. A second order system, for example, has a natural frequency $\left(w_{n}\right)$ which reflects its dynamic response characteristics. Actuator commands generated by the system, in response to a desired state trajectory, also give us a strong indication of the bandwidth of the system. Plots of control action with and without the inertial augmentation indicate that the bandwidth available to JASON JUNIOR is larger when the inertial measurement augment the acoustic position system. The improvement is most striking for the case of low acoustic update rate and high acoustic noise. 
As a tracker, the 'hybrid' state estimator was similarly more successful than the unaugumented observer in reaching the actual state within a specified tolerance in a shorter period of time. As expected, the estimated position and velocity errors, and actual position and velocity errors were smaller for the augmented system. Here also the improved tracking was most pronounced in the cases of large acoustic noise levels. The differences were quite significant when acoustic update rate was relatively low and noise level was high.

In general the 'hybrid' state estimator performed well. In fact, there never appeared to be a situation where some improvement was not shown. 'Flyers' were not simulated and would have shown another advantage to the estimator. On the other hand, growth in accelerationderived position error was not simulated either. Although not modeled in the simulation, data analysis indicated stability in acceleration-derived position for as long as 60 seconds (the length of several of the data runs.)

\subsubsection{The Effects of the JASON 'Hybrid' Estimator}

Unlike the case of JASON JUNIOR there is no direct comparison to determine the incremental improvement in bandwidth. Because of the ranges that the RF system is capable of tracking, the acoustic position measurement system would probably not even be fielded in similar conditions. This increase in range capability, if successful, represents a quantum jump over existing acoustic systems. The real question is whether the combined measurements will retain sufficient accuracy to allow closed loop control of the vehicle. The answer is a qualified yes. The qualifications are the assumptions that we made at the beginning of our investigation in Chapter 6 and were part of the development of our simulation.

\subsection{Refinements and Improvements}

\subsubsection{Strapdown Inertial Measurement Systems}

As more experience is gained with the strapdown systems in more commonplace applications, the demand for better and cheaper sensors will grow. New technology is being applied to sensors which will allow much higher accuracy in inertial measurements.

One example of improved sensors is the Ring Laser Gyro. Currently too expensive for our testing, it may soon prove to have a high enough performance to cost ratio and be packaged 
small enough to be included in a package such as the SIMS [22]. Incremental improvements in the pendulous accelerometer continue to be made also, resulting in the availability of lower cost, higher performance accelerometers for low to medium cost systems.

As sensors improve a need exists to improve the circuitry that transports the information to the computer. Lower circuit noise and higher $A / D$ resolution will be required to take advantage of any new gains in sensor sensitivity or reductions in sensor noise.

\subsubsection{Mobile Acoustic Nets}

One of the major drawbacks of the Mobile acoustic net developed for JASON is the current inability to extend its use to deep water. The angular separation of the acoustic transducers is so small when the ROV is deep (greater than 1000 meters) that the mobile system cannot adequately track the ROV. The only parameter that can be resolved well at deep depth is depth itself. Unfortunately, pressure sensors can provide this information nearly equally as well.

Tracking in open ocean may also suffer similar problems, if there are no sites to mount the slave stations. Conceivably, we could take advantage of a high data rate satellite navigation system such as GPS. Such a marriage would have its own set of problems and still be limited to relatively shallow water, however.

The problem of tracking at depth, brings us back to the need for tracking between acoustic fixes (from a bottom mounted acoustic net, for example) for an extended period of time. The SIMS ultimately becomes a sensor whose importance grows with the ambitiousness of the tasking, especially in a deep submergence application.

\subsection{Remaining Work}

As indicated in the preceding paragraphs, additional improvements are not only possible, but necessary for improved performance. Sensors must not only be cleaner and more sensitive, but be used to support one another. Including larger numbers of independent sensors can only help in offsetting the difficulties of deep, open ocean work. 


\subsubsection{Control Developments}

\section{Trajectory Specification}

The concentration thus far has been on the geographic positioning of ROV's with regard only to heading when it comes to attitude. This has not been due to the laziness of the control engineer, but rather because of a lack of attitude sensors and the likelihood that even if the attitude were observable, the ROV would probably not be controllable in attitude. With larger ROV's with more thrusters, such as JASON, coming on line, and with cleaner, higher update rate attitude sensors, it will soon be possible to control vehicles in attitude. Initially, control may only be the maintenance of a level attitude, as perhaps in the case of JASON, for which they are already statically stable. Just a reliably level platform can open the door to a wide range of applications.

Eventually, an arbitrary attitude may be specified, within reasonable limits, as part of a trajectory profile. A vehicle which is controllable to an arbitrary attitude may challenge our conventional thoughts about ROV static stability or thruster power requirements.

\section{Adaptive Modeling}

Still in the controls area, but with an emphasis on better modeling, is the need to convert the work in hybrid state estimation into the nonlinear modeling arena. Nonlinear modeling of the vehicle dynamics may significantly assist the more ambitious trajectory specification problem outlined previously.

The logical, though by no means trivial, extension to nonlinear modeling is the use of adaptive control. If for no other reason than model refinement, the employment of adaptive techniques should ensure the success of more ambitious mission tasking.

Some examples of more ambitious tasking are presented in the next section.

\subsubsection{Applications}

\section{Autonomous Vehicles}

An extension of the far-ranging ROV is the autonomous ROV. As in the case of the tethered vehicle, the same sort of problems in navigation and state determination need to be solved 
to make the autonomous vehicle successful. Fortunately, the methods developed here are not restricted in their application to tethered vehicles.

\section{Level Platform Applications}

Once the vehicle is controllable in three dimensional position and can maintain a level attitude, a number of applications for this technology cam be envisioned. Immediately, the idea of a stabilized side-scan sonar comes to mind. Very high precision bathymetry or geological surveys can be the result of a stabilized ROV with an altimeter or sidescan sonar. As well, many optical sensors would benefit from the additional stability. The real question, as with strapdown systems is: is it more effective to stabilize the platform or do post-processing of the sensor data? This depends on the nature of the available sensor data and is an appropriate question for future research.

\section{Controllable Attitude Applications}

The next level of improvement is the arbitrary and dynamic placement of the vehicle in attitude. This may be particularly suited for contour following, such as in the inspection of the hull of a ship. Any application which requires that a vehicle- borne sensor be held vertical to the surface of interest would be an appropriate user of this capability. As with most things, though, the applications often follow the proof of the technology and may not be fully explored for several years.

\subsection{Closing Remarks}

Clearly, these last several paragraphs have raised more questions than they have answered. New capabilities often challenge our way of thinking and beg for more work to clearly define the issues and assess their impact.

We do know that appropriate tailoring of state estimation techniques to utilize the available sensors and fit the specific circumstances can improve the automatic control of Remotely Operated Vehicles.

The results are encouraging and were worth the effort. 


\section{Bibliography}

[1] P. D. Rushfeld, A. Conter, and D. L. McLeod. State-of-the-art rov and control system for deepwater pipe repair. In Offshore Technology Conference 1987 Proceedings, Vol 1, June 1978.

[2] Francis B. Hildebrand. Advanced Calculus for Applications. Prentice-Hall, Inc., Englewood Cliffs, NJ, second edition, 1976.

[3] Dana Yoerger, James Newman, and Jean-Jacques E. Slotine. Supervisory control system for the jason rov. In IEEE Journal of Oceanic Engineering, VOL OE-11, No 3., July 1986.

[4] S. F. Schmidt. Strapdown inertial systems - theory and applications. In AGARD Lecture Series No.95: Strap-Down Inertial Systems, NATO Advisory Group for Aerospace Research \& Development, May 1976.

[5] John N. Newman. Marine Hydrodynamics. MIT Press, Cambridge, MA, fifth edition, 1986.

[6] R. Sabersky, A. Acosta, and E. Hauptman. Fluid Flow: A First Course in Fluid Mechanics. Macmillan Publishing Co., Inc., New York, second edition, 1971.

[7] J. Feldman. DTNSRDC Revised Standard Submarine Equations of Motion. Technical Report DTNSRDC/SPD-0393-09, David Taylor Naval Ship R\&D Center, 1979.

[8] Katsuhiko Ogata. Discrete-Time Control Systems. Prentice-Hall, Inc., Englewood Cliffs, $\mathrm{NJ}, 1987$. 
[9] Karl J. Astrom and Bjorn Wittenmark. Computer Controlled Systems; Theory and Design. Prentice-Hall, Englewood Cliffs, NJ, 1984.

[10] Gene F. Franklin. Digital Control of Dynamic Systems. Addison-Wesley, Reading, MA, 1980.

[11] Bernard Friedland. Control System Design; an Introduction to State Space Methods. McGraw-Hill, New York, NY, 1986.

[12] David G. Luenberger. Introduction to Dynamic Systems. John Wiley and Sons, New York, 1979.

[13] Arthur Gelb, editor. Applied Optimal Estimation. MIT Press, Cambridge, MA, 1974.

[14] S. M. Bozic. Digital and Kalman Filtering. Edward Arnold, 41 Bedford Square, London, 1979.

[15] P. G. Savage. Strapdown sensors. In AGARD Lecture Series No.95: Strap-Down Inertial Systems, NATO Advisory Group for Aerospace Research \& Development, June 1978.

[16] J. R. Catford. Application of strapdown inertial systems with particular reference to underwater vehicles. In AGARD Lecture Series No.95: Strap-Down Inertial Systems, NATO Advisory Group for Aerospace Research \& Development, June 1978.

[17] Frank B. Hill. A Study of Coordinte-Conversion Errors in Strapped-down Navigation. Technical Report ESL-R-244, Electronic Systems Laboratory, MIT, 1965.

[18] Charles Broxmeyer. Inertial Navigation Systems. McGraw-Hill, New York, 1964.

[19] George R. Pitman. Inertial Guidance. John Wiley and Sons, New York; 1962.

[20] A. VanBronkhorst. Strapdown system algorithms. In AGARD Lecture Series No.95: Strap-Down Inertial Systems, NATO Advisory Group for Aerospace Research \& Development, June 1978.

[21] Robert Grover Brown. Introduction to Random Singal Analysis and Kalman Filtering. John Wiley and Sons, Inc, New York, 1983. 
[22] Paul G. Savage. Advances in strapdown sensors. In AGARD Lecture Series No.139: Advances in Strapdown Inertial Systems, NATO Advisory Group for Aerospace Research \& Development, May 1984. 


\section{Appendix}

Attached is the PC MATLAB code for the JASON ROV hybrid observer. PC MATLAB uses its own high level programming language to implement its featured functions. These user created macro files use the .M extension.

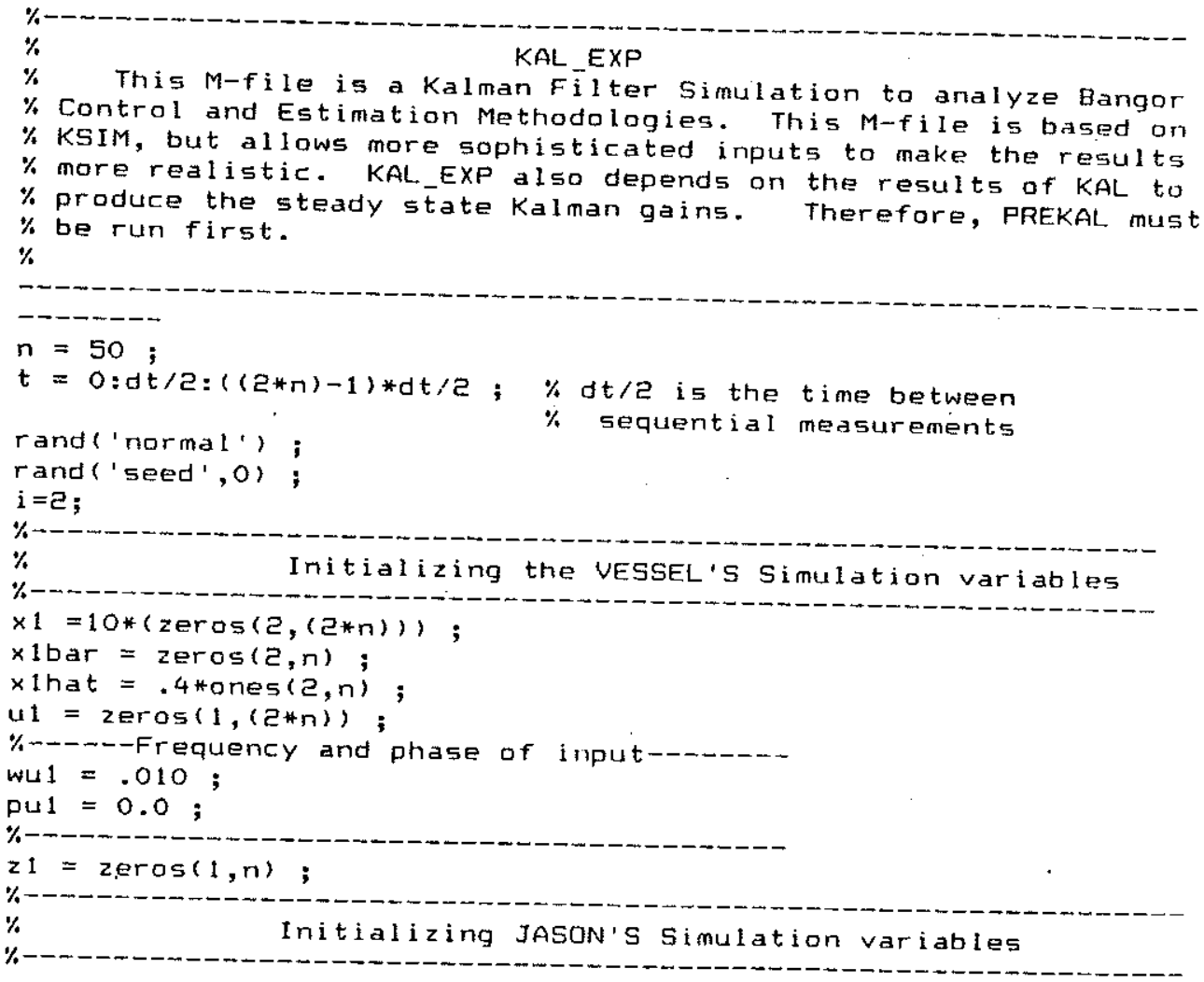




\section{Appendix}

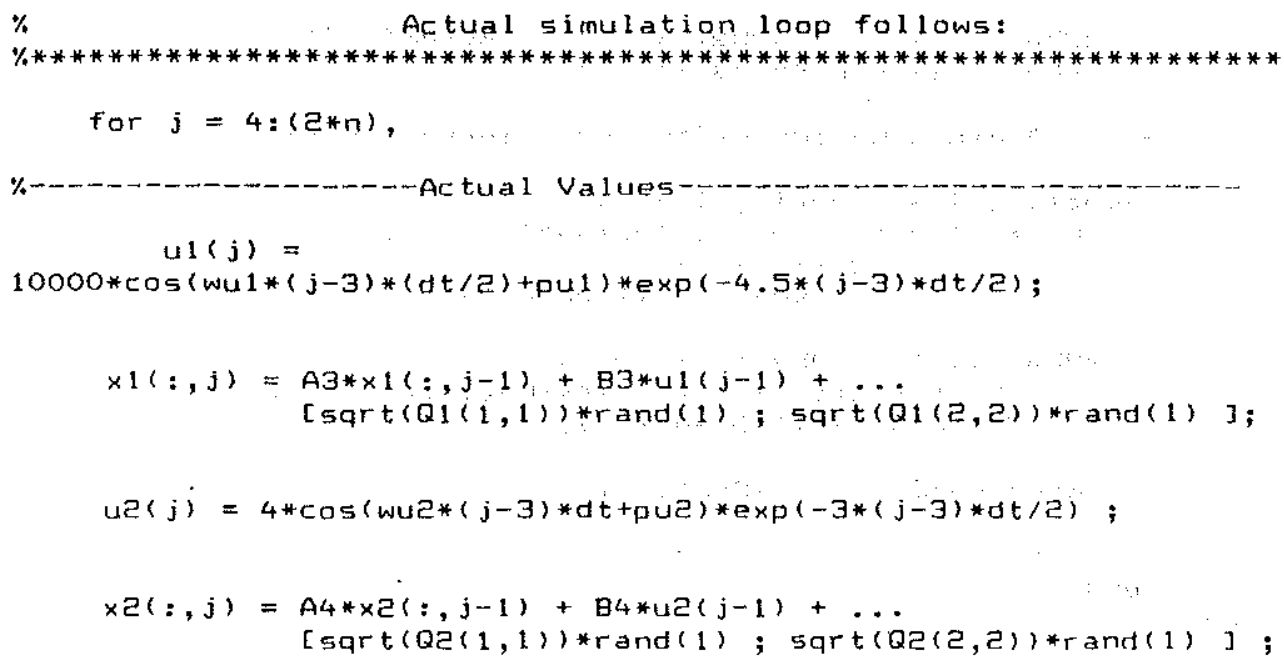




\section{Appendix}

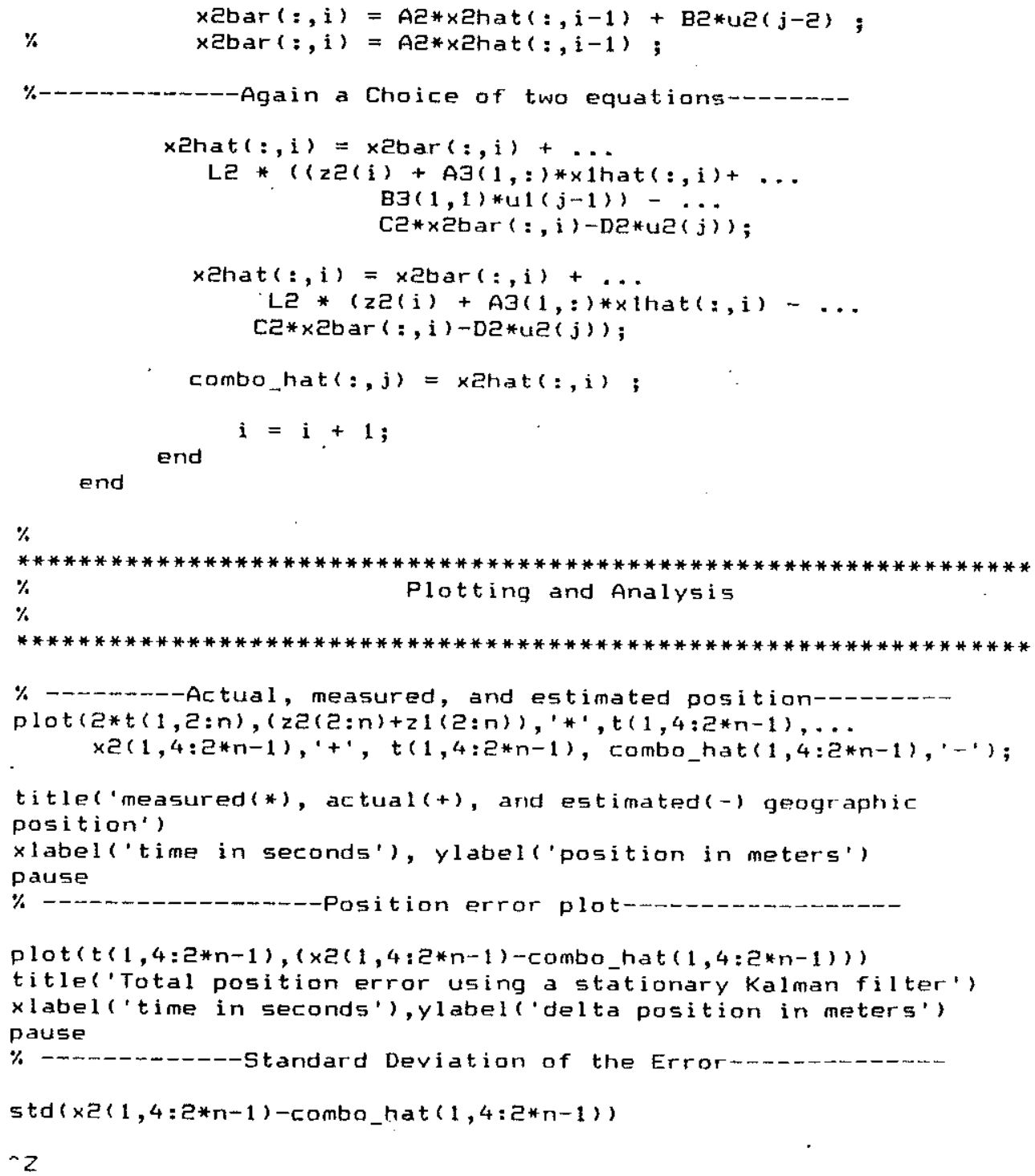

